University of Louisville

ThinkIR: The University of Louisville's Institutional Repository

Electronic Theses and Dissertations

$12-2014$

\title{
Extracellular matrix proteins and the liver-lung axis in disease.
}

Veronica L. Massey
University of Louisville

Follow this and additional works at: https://ir.library.louisville.edu/etd

Part of the Pharmacy and Pharmaceutical Sciences Commons

\section{Recommended Citation}

Massey, Veronica L., "Extracellular matrix proteins and the liver-lung axis in disease." (2014). Electronic Theses and Dissertations. Paper 1752.

https://doi.org/10.18297/etd/1752

This Doctoral Dissertation is brought to you for free and open access by ThinkIR: The University of Louisville's Institutional Repository. It has been accepted for inclusion in Electronic Theses and Dissertations by an authorized administrator of ThinkIR: The University of Louisville's Institutional Repository. This title appears here courtesy of the author, who has retained all other copyrights. For more information, please contact thinkir@louisville.edu. 


\title{
EXTRACELLULAR MATRIX PROTEINS AND THE \\ LIVER-LUNG AXIS IN DISEASE
}

By

\author{
Veronica L. Massey \\ B.S. Xavier University, 2010 \\ M.S., University of Louisville, 2012
}

\author{
A Dissertation \\ Submitted to the Faculty of the \\ School of Medicine of the University of Louisville \\ In Partial Fulfillment of the Requirements \\ for the Degree of \\ Doctor of Philosophy \\ Department of Pharmacology and Toxicology \\ University of Louisville \\ Louisville, KY
}

December 2014 



\title{
EXTRACELLULAR MATRIX PROTEINS AND THE \\ LIVER-LUNG AXIS IN DISEASE
}

\author{
By \\ Veronica L. Massey \\ B.S., Xavier University, 2010 \\ Thesis Approved on \\ $11 / 03 / 2014$ \\ by the following Thesis Committee:
}

\begin{tabular}{c}
\hline Gavin E. Arteel, Ph.D. \\
\hline Jesse Roman, M.D. \\
\hline Leah Siskind, Ph.D. \\
\hline Aruni Bhatnagar, Ph.D. \\
\hline J. Christopher States, Ph.D.
\end{tabular}




\section{DEDICATION}

This dissertation is dedicated to my parents

Dr. Janet Lynch

And

Dr. Evan Massey

for their constant encouragement and support

In all my endeavors. 


\section{ACKNOWLEDGEMNETS}

First and foremost I would like to thank my mentor for his complete support and his steadfast confidence in my abilities as a scientific researcher since the beginning. His encouragement during my first scientific investigations as an undergraduate researcher and throughout my graduate career has been instrumental in the success of my research. I would also like to thank my dissertation committee members, Drs Jesse Roman, Leah Siskind, Christopher States, and Aruni Bhatnagar for their guidance and support. I would like to thank Dr. Michael Merchant and Danny Wilkey for the LC-MS work. I would also like to thank Dr. Shirish Barve for his help with the deep sequencing and Kendall Stocke for his help analyzing the metagenomics data. I would also like to thank Dr. Roman's group for their expertise in working with the lung, especially Edilson Torres who was appreciated for his help in isolating and preparing the lungs, and Jeff Ritzenthaler who was appreciated for his guidance, particularly in pulmonary immunohistochemistry.

Many thanks to my lab mates who have been an instrumental part of my graduate experience both at and away from the bench: Lauren Poole, for her help with animal husbandry and her listening ear during times of trouble; Robin Schmidt, for her expertise in animal handling as well as her wise words and witty humor; Nikki Warner for her assistance with sacrifices and her bubbly personality 
which always kept us going; Helen Tan, for her assistance with animal husbandry; Philip Kaiser, for his training and patience with me as undergraduate researcher; and also to Jule Beier, for her many hours of training at the bench and especially for her expertise in animal handling and in vivo models. A special thanks goes out to my lab moms - Jenny Jokinen and Deanna Siow - who have been a constant source of help and encouragement at the bench and beyond.

Finally, I would also like to thank my friends and family for their support through my years in graduate school. I would especially like to thank Lauren Poole, Glenn Vicary, Nikki Warner, and Gretchen Holz for their friendship both in and outside the lab. I would like to thank Amir Sheth for his artistic contributions to this work, but most importantly for his endless patience, encouragement and science inspired puns. Finally, many thanks to my family - Jennie Lynch, Evan Massey, and Blair Ferrell - for all of their loving support. 


\section{TABLE OF CONTENTS}

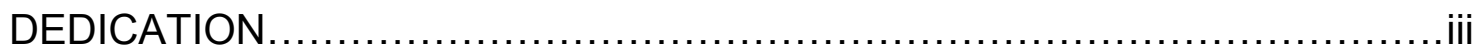

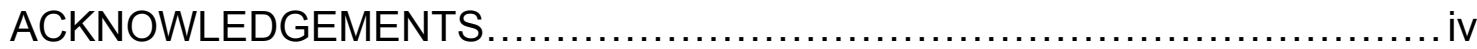

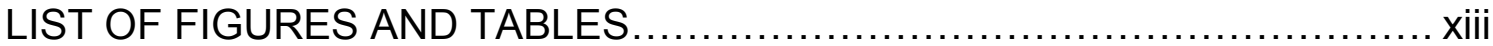

\section{CHAPTER}

\section{INTRODUCTION}

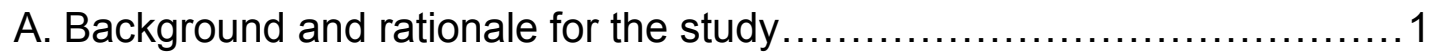

1. The extracellular matrix: more than a scaffold ....................... 1

2. The role of ECM in inflammation and disease $\ldots \ldots \ldots \ldots \ldots \ldots \ldots \ldots . \ldots \ldots$

3. Statement of goals................................................. 4

4. Natural history of liver disease ......................................

5. Alcoholic liver disease .............................................. 6

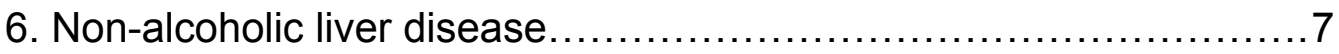

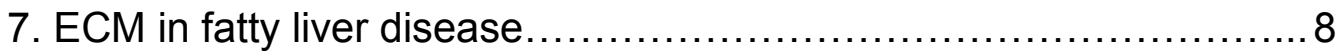

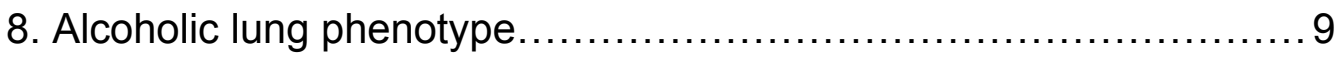

9. Parallel mechanisms of injury in the liver and lung.................... 10

10. The gut-liver-lung axis............................................... 11

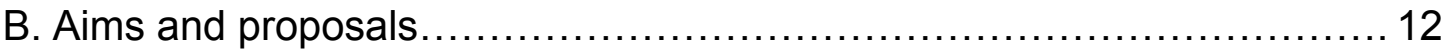

1. Protective effects of prebiotics in the setting of non-alcoholic liver

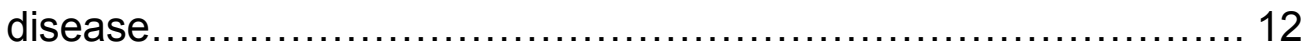

2. Characterization of a model of concomitant liver and lung injury.......13 
3. Can we characterize the hepatic extracellular proteome?.

II. EXPERIMENTAL PROCEDURES ................................... 17

A. Animals and treatments.................................................. 17

1. Arsenic, high fat diet, and oligofructose feeding...................... 18

2. Chronic model of alcohol exposure ...................................18

3. Etanercept administration .......................................... 19

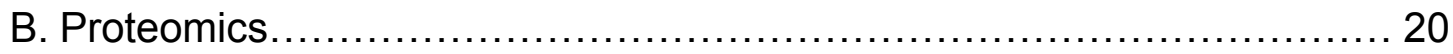

1. 3-step ECM extraction.......................................... 21

2. Sample cleanup and preparation for liquid chromatography ..........23

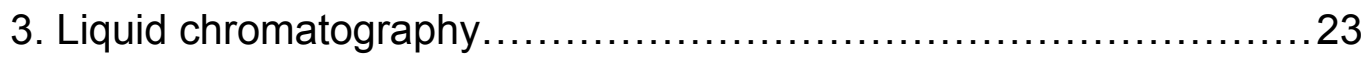

4. Mass spectroscopy .................................................. 24

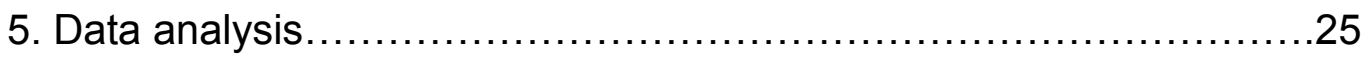

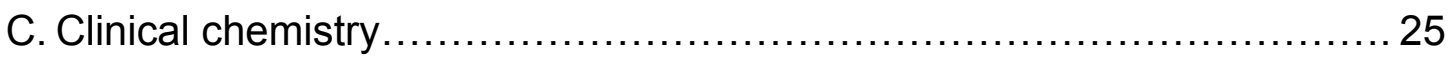

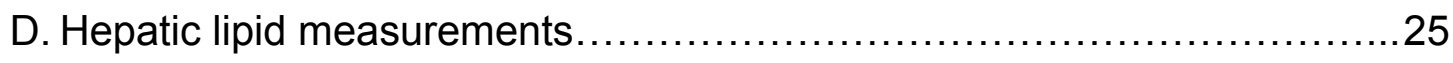

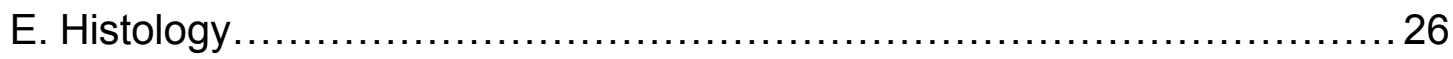

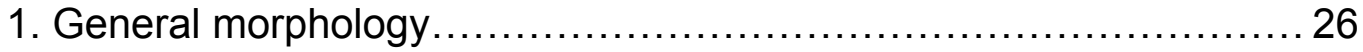

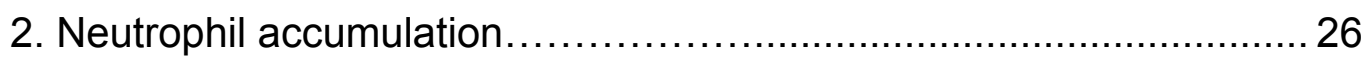

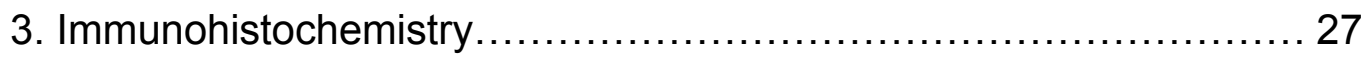

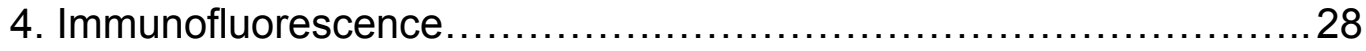

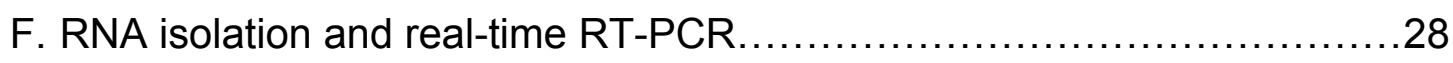

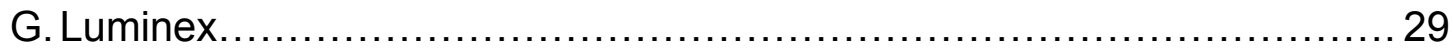

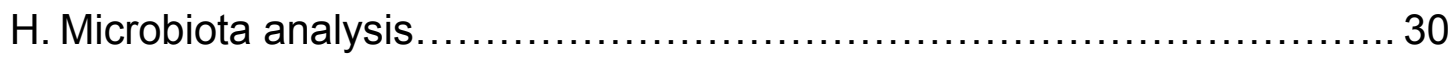

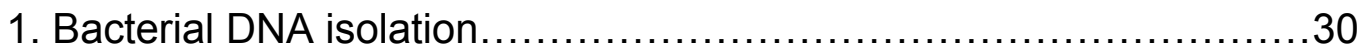




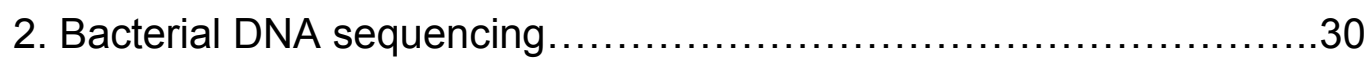

3. Bacterial DNA analysis .............................................. 31

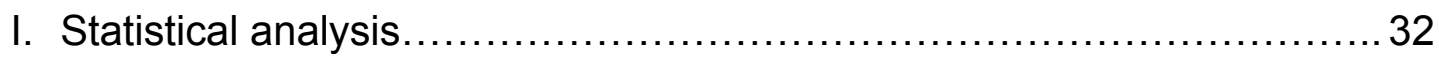

III. PROTECTION AGAINST ENHANCED LIVER INJURY CAUSED BY ARSENIC IN A MODEL OF HIGH FAT DIET INDUCED NON-ALCOHOLIC STEATOHEPATITIS - ROLE OF THE ECM............................ 34

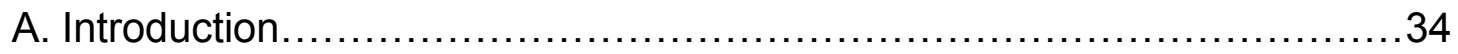

B. Experimental procedures............................................... 37

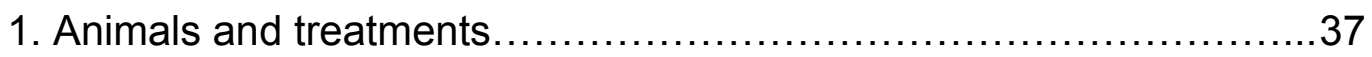

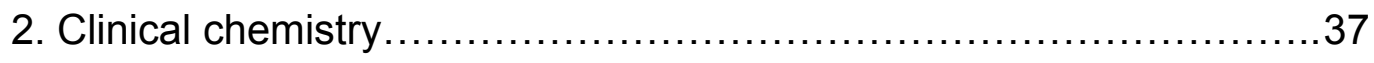

3. Lipid determination................................................. 37

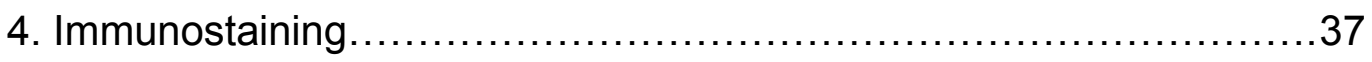

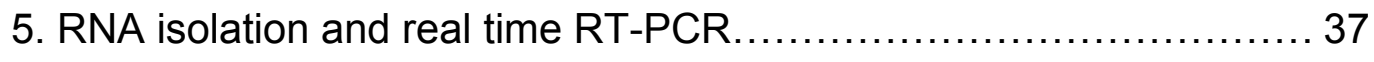

6. Cecal DNA extraction............................................... 37

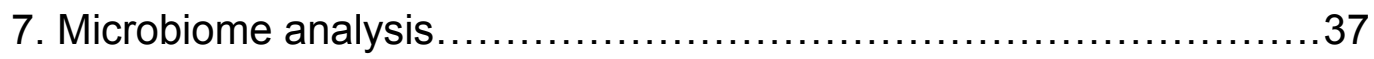

8. Statistical analysis................................................ 37

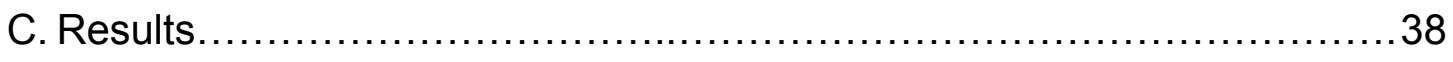

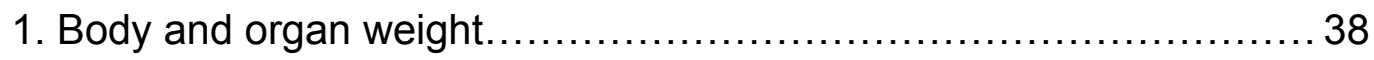

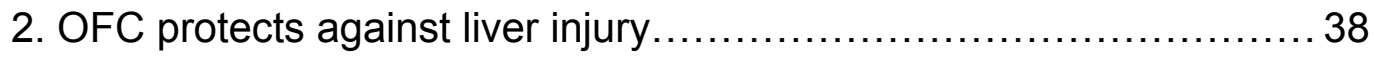

3. OFC protects against liver inflammation...............................43

4. OFC protects against fibrin deposition............................... 46

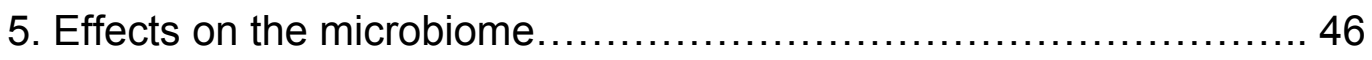

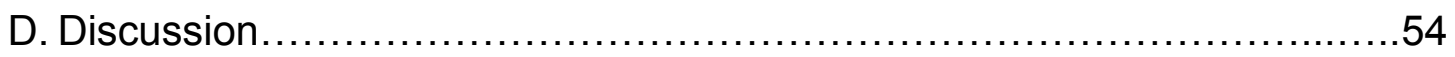


IV. CHARACTERIZATION OF THE LINK BETWEEN THE LIVER AND LUNG IN THE SETTING OF ALCOHOL EXPOSURE - THE ROLE OF TNFa... 58

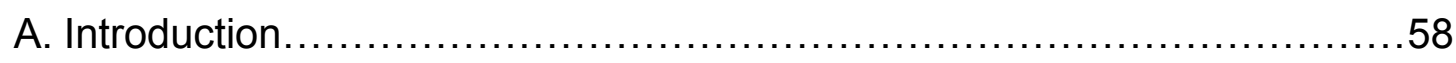

B. Experimental procedures.................................................... 61

1. Animals and treatments.............................................. 61

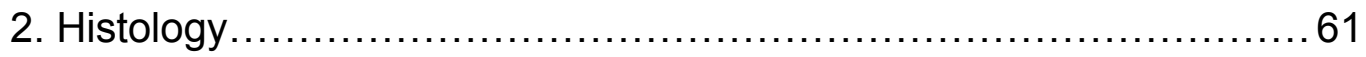

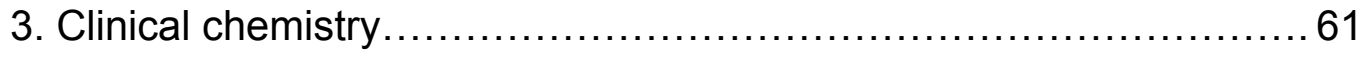

4. RNA isolation and real time RT-PCR .............................61

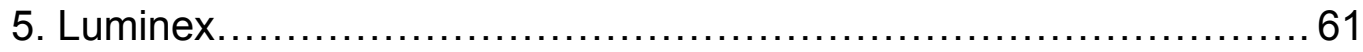

6. Statistical analysis................................................ 61

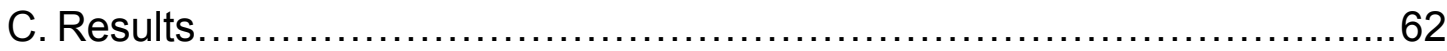

1. Ethanol induced steatosis and enhanced LPS-induced liver injury....62

2. Ethanol enhanced LPS-induced lung injury and inflammation.........65

3. Ethanol differentially alters cytokine/chemokine expression in the liver

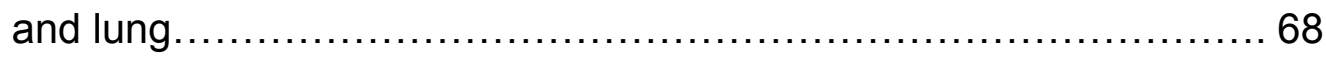

4. Effect of etanercept on indices of injury and inflammation.............71

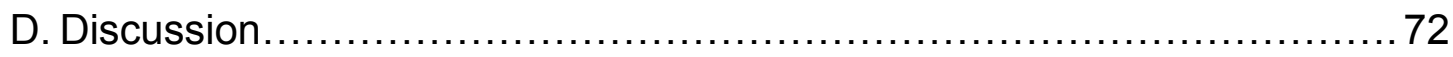

V. EFFECTS OF ETHANOL AND LPS ON THE HEPATIC EXTRACELLULAR

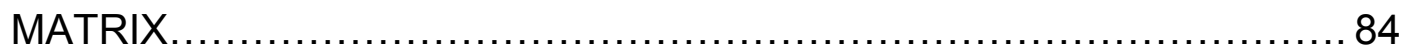

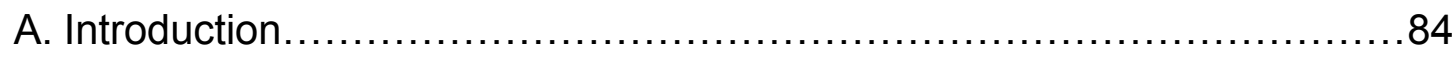

B. Experimental procedures............................................... 86

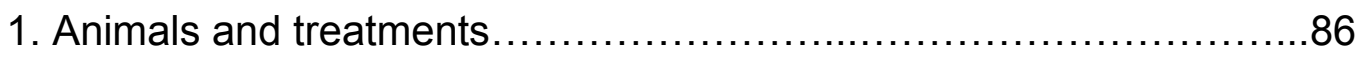

2. ECM extraction....................................................... 86 


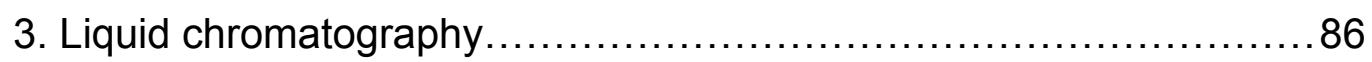

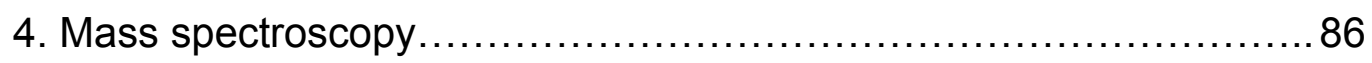

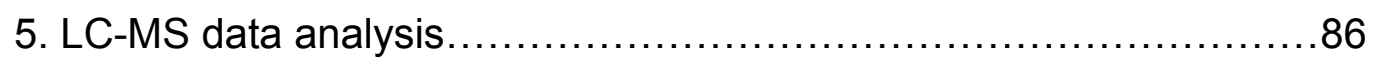

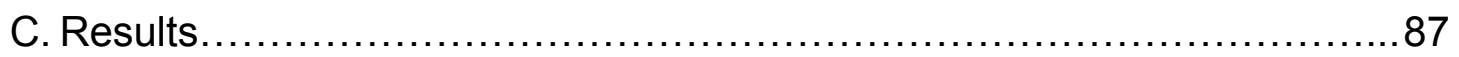

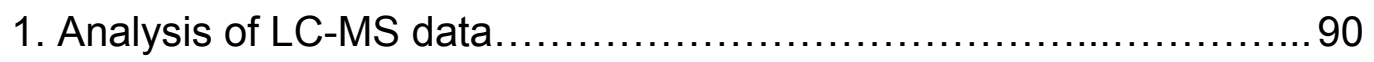

2. 3-step serial extraction creates 4 extracts with distinct protein

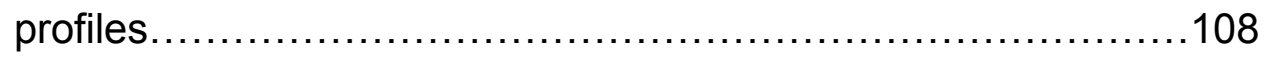

3. Qualitative changes to the extracellular proteome in response

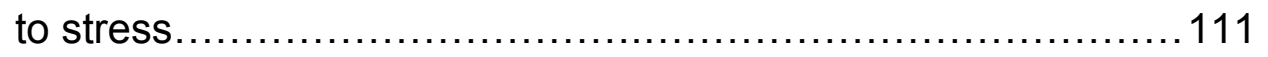

4. Quantitative changes to the extracellular proteome caused by stress.......................................................... 115

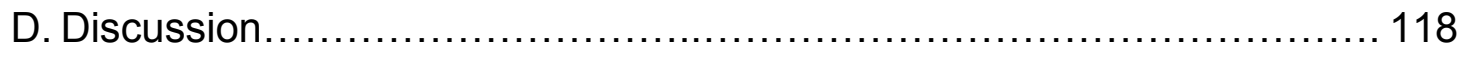

VI. DISCUSSION AND CONCLUSIONS................................. 126

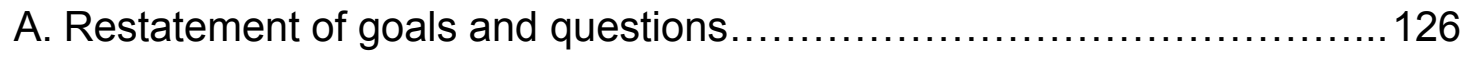

B. Major findings of this dissertation................................... 127

1. Dysbiosis contributes to hepatic sensitization and transitional tissue

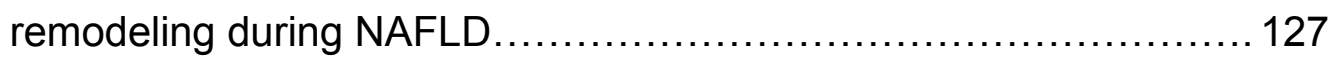

2. Chronic ethanol exposure causes unique proinflammatory changes in the liver and lung after systemic LPS administration................. 129

3. The hepatic matrisome undergoes dynamic changes after ethanol and LPS exposure ................................................. 131

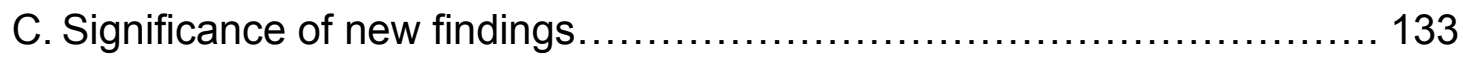

D. Strengths and weaknesses of this dissertation.......................... 135 


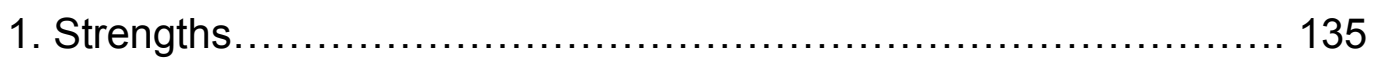

2. Weaknesses....................................................... 136

E. Future directions....................................................... 138

1. Does HFD-induced dysbiosis change arsenic metabolism and/or

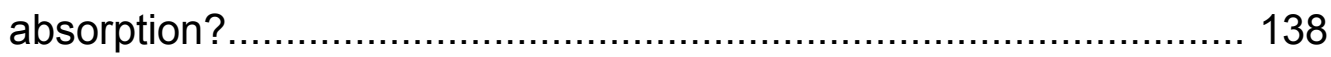

2. Does liver-derived TNFa contribute to the alcoholic

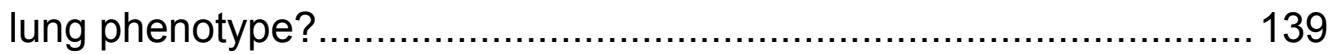

3. What are the functional effects of changes in the hepatic matrisome of LPS and ethanol-exposed liver?................................................ 140

4. What changes does the alcohol exposure cause to the

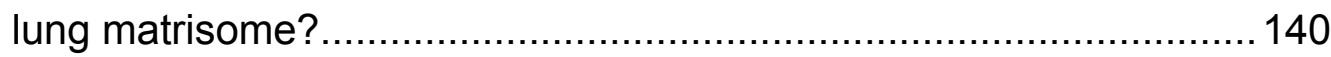

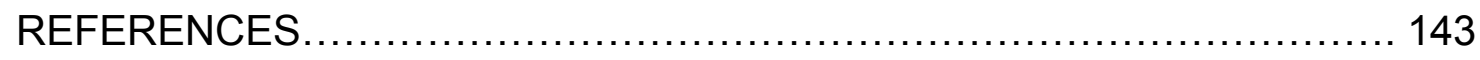

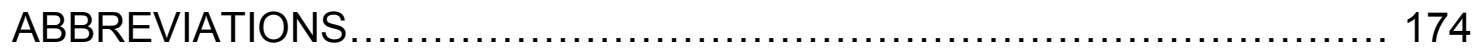

CURRICULUM VITAE .................................................... 176 


\section{LIST OF FIGURES}

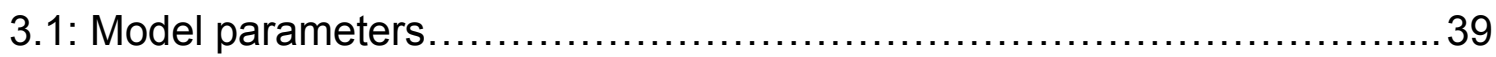

3.2: OFC protects against liver injury and steatosis......................... 41

3.3: OFC protects against hepatic inflammation............................. 44

3.4: OFC protects against sinusoidal fibrin accumulation $\ldots \ldots \ldots \ldots \ldots \ldots \ldots \ldots . \ldots \ldots$

3.5: Effects of HFD, arsenic, and OFC on bacterial abundance and diversity ...50

3.6: HFD, arsenic, and OFC exposure caused unique changes to the microbiome

4.1: Effects of chronic ethanol pre-exposure on LPS induced liver injury and inflammation

4.2: Effects of chronic ethanol pre-exposure on LPS induced lung injury and inflammation

4.3: Effects of chronic ethanol exposure on LPS-induced cytokine

expression.

4.4: Etanercept protects against enhanced cytokine levels in the BAL without concomitant protection against liver injury

4.5: Proposed mechanism of interdependent injury.

5.1: Scheme of extraction methodology 88

5.2: Liver extracts have unique protein profiles based on fraction type 
and experimental group.......

5.3: Ethanol and LPS cause dynamic changes in the hepatic matrisome........112

5.4: Liver extracts show quantitative changes in the hepatic matrisome........116 


\section{CHAPTER I}

\section{INTRODUCTION}

\section{A. Background and rationale for this study}

\section{The extracellular matrix: more than a scaffold}

The extracellular matrix (ECM) forms a complex microenvironment in the extracellular space and is critical for development, tissue structure, and cellular response. The ECM consists of a myriad of proteins, glycoproteins, and proteoglycans that form a cross-linked scaffold that surrounds cells in all solid organs (1). These fibrous proteins are produced by cells (i.e., fibroblasts) and, upon secretion into the extracellular environment, crosslink together to create a meshwork that serves as a scaffold for cells and that provides structure to tissue. The collagen scaffold to which glycoproteins and proteoglycans adhere is the main characteristic shared by the ECM of the basement membranes and interstitial matrices, regardless of physiological domain (1). Physical changes to the extracellular landscape, including changes in ECM topography, crosslinking, and biochemistry, can affect cell migration, adhesion, and activation (2). In addition to their role as structural compounds, these proteins can also serve as signaling molecules. Indeed, ECM proteins are ligands of cell surface receptors, including integrins, which bind specific ECM matrix protein domains (e.g., the RGD motif). The ECM also contributes to cell signaling by binding soluble 
signaling molecules including cytokines, chemokines, and growth factors to create signaling gradients required for tissue development and migration of inflammatory cells during acute inflammation (3).

Proteases and protease inhibitors comprise another group of proteins critical in the extracellular space. These enzymes play an important role in the homeostasis of the extracellular environment and contribute to ECM remodeling in disease states (4). Proteases may enhance the deposition of proteins to the ECM (e.g., proteases involved in the complement and coagulation cascades). Proteases can also break down matrix components to allow for rapid changes in the ECM organization, such as matrix metalloproteinases (MMPS), a disintegrin and metalloproteinase with thrombospondin motifs (ADAMTS), and other serine proteases. The effects of ECM degradation by proteases modify both the physical and biochemical environment of the extracellular space. For example, physical dissolution of the ECM by proteases can create space through which cells can migrate (5). Alternatively, cleavage of ECM proteins by proteases can alter cell-ECM interactions by exposing/removing cryptic binding sites; for example, proteolysis of laminin-5 reveals integrin-binding sites which are critical for cell adhesion (6). Proteases can also contribute to ECM dynamics by modulating the activity of other enzymes (e.g., proteinase precursors (7) and soluble mediators (e.g., IL-8, (2)). Because of the wide-reaching effects of proteases, their activity is regulated and maintained via complex, often redundant, mechanisms. Indeed, in addition to regulation of rates of synthesis and secretion, extracellular proteases frequently require activation, often via 
cleavage by another protease, and conversely can be inhibited by endogenous inhibitors such as tissue inhibitors of matrix metalloproteinases (TIMPs) (5).

\section{The role of the ECM in inflammation and disease.}

Tight regulation of the ECM composition is necessary for normal tissue homeostasis, and dysregulation is often a hallmark in tissue pathology. Therefore, the organization, composition, and orientation of the ECM are controlled via multiple mechanisms including protein synthesis, modification, excretion, crosslinking, and enzymatic degradation. Such tight regulation on so many levels also permits rapid changes in the ECM that allow it to play a dynamic and responsive role during inflammation and tissue repair.

Perturbations in the ECM during either of these physiological states can contribute to disease initiation and development.

During an acute inflammatory response, the ECM plays a pivotal role by serving as a structural barrier and chemokine reservoir, thereby regulating inflammatory cell migration. Indeed, studies have shown that neutrophil transmigration is greater through vascular areas with lower ECM concentration (8). Additionally, ECM binding of cytokines, including IL-2, TNFa, TGF $\beta$, and RANTES (9-11), have been shown to regulate cell activation, adhesion, and migration. The interaction between the ECM and cell infiltration is bidirectional as leukocytes integrate structural and biochemical signals from the ECM, they in turn release matrix-degrading proteases (12) which alter the extracellular composition and allow for easier cell migration. 
In the setting of chronic inflammation, dysregulation of ECM composition can lead to pathological tissue remodeling and, if not resolved, disease states including hepatic $(13,14)$ and pulmonary fibrosis $(15-17)$, atherosclerosis $(18$, 19) and cancer (20, 21). Initiation of these disease states can occur via dysregulation at the level of ECM synthesis and/or degradation, leading to altered matrix accumulation and modification. For example hepatic fibrosis is characterized by a robust increase in collagen la1 secretion by hepatic stellate cells (HSCs) (22) combined with a concomitant increase in crosslinking and a decrease in enzymatic degradation (23). Such dynamic changes in extracellular composition can initiate and perpetuate disease via a variety of mechanisms including altered tissue biomechanics (i.e. tissue stiffness) $(24,25)$, increased integrin-mediated cell adhesion (26) and activation of immune cells (27).

\section{Statement of goals}

The role of tissue remodeling in advanced stages of liver disease, including fibrosis, is clear. Indeed, the major macroscopic change in hepatic fibrogenesis is characterized by severe collagen deposition and scarring. Currently, hepatic fibrosis is generally considered irreversible, and there is currently no FDAapproved therapy to halt or reverse liver fibrosis. Recent studies have shown that transitional tissue remodeling may occur during inflammatory liver disease, before the development of fibrosis (28). Additionally, protecting against these subtle changes can protect against liver injury and inflammation (29). These findings suggest that targeting the ECM in earlier stages of liver disease could 
provide therapeutic benefit by preventing disease progression to later, irreversible stages of fibrosis. The goal of this dissertation is to examine the role of transitional tissue remodeling in fatty liver disease. Furthermore, it aims to identify new extracellular matrix proteins that play a role in early stages of remodeling which could serve as novel therapeutic targets.

\section{Natural history of liver disease}

The natural history of liver disease is well documented and encompasses a spectrum of disease states including earlier stages of fatty liver disease such as steatosis, steatohepatitis, and later stages of disease including fibrosis and cirrhosis $(30,31)$. The earliest stage of liver disease, simple steatosis, is characterized by micro- and macro-vesicular fat accumulation and is generally considered reversible if appropriate changes to the lifestyle are made and maintained. In the absence of lifestyle modifications, individuals may develop steatohepatitis, which is characterized by persistence of accumulated fat in addition to chronic hepatic inflammation (hepatitis). Later stages of liver disease, including fibrosis and cirrhosis, are characterized by collagen deposition and regenerative nodules, respectively $(31,32)$.

Despite the vast amount of money spent on treating alcoholic liver disease, therapeutic options remain limited to palliative care in the late stages of disease. Currently there is no FDA-approved therapy in existence to halt or reverse disease progression. If a rational, targeted therapy is to be developed, currently 
accepted mechanisms of disease progression must be better understood and new therapeutic targets identified.

\section{Alcoholic liver disease}

Alcohol is one of the most commonly recognized causes of liver disease. Alcoholic liver disease (ALD) affects more than 10 million Americans each year at a cost of more than $\$ 166$ billion annually to treat the medical consequences of this disease (33). After absorption from the small intestine, milli-molar concentrations of ethanol flow through the portal circulation and to the liver for degradation making the liver a prime target of ethanol toxicity. During oxidative metabolism of alcohol in hepatocytes by alcohol dehydrogenase (ADH) and CYP2E1, a robust increase in the NADH/NAD+ ratio lends to a more reduced cellular environment. This reduced microenvironment blocks the beta-oxidation of fatty acids in the liver and increases the esterification of fatty acids. Accumulation of excess fatty acids in the liver leads to storage of these lipids in lipid droplets known as steatosis, which is a hallmark of early liver disease.

Steatosis develops in nearly all individuals who consume moderate amounts of alcohol, and for most people has no health consequences. Indeed, at the organismal level, hepatic steatosis may be viewed as a protective mechanism, as it restricts plasma lipid levels from rising. However chronic alcohol consumption can lead to development of later stages of liver disease, including alcoholic steatohepatitis (ASH) as well as fibrosis and cirrhosis. These more severe stages of ALD develop in only a minority of chronic alcoholics. One 
explanation for this discrepancy is the 2-hit hypothesis, which suggests that progression from simple steatosis to later stages of liver disease requires multiple insults, or 'hits' $(34,35)$. According to this paradigm, a first 'hit,' such as hepatic steatosis, sensitizes the liver to a second hit such as oxidative stress (36) or inflammatory cytokine signaling (37), both of which are also increased by ethanol consumption.

\section{Non-alcoholic fatty liver disease (NAFLD)}

Another common cause of fatty liver disease is obesity. Obesity is a growing problem both nationally and globally, with statistics estimated that more than 78 million U.S adults are clinically obese (38). Obesity is associated with a myriad of health effects including metabolic syndrome, cardiovascular risks, and nonalcoholic fatty liver disease (NAFLD). It has been difficult to estimate the prevalence of NAFLD within populations and figures range depending on the cohort studied, however current estimates indicate that up to $19 \%$ of the American population may have some degree of NAFLD (39), with the highest prevalence of NAFLD among the morbidly obese (40). Interestingly, despite a high prevalence of steatosis among obese individuals, it is estimated that less one third of patients with NAFLD will develop nonalcoholic steatohepatitis (NASH) (40-42), the more severe inflammatory stage of the disease. Increased susceptibility to disease progression in some individuals is likely due to a variety of factors, including genetic predisposition, and environmental exposures (43). 
Similar to ALD, it is hypothesized that multiple hits are required for the development of later stages of $\operatorname{NAFLD}(34,43)$.

Our lab is particularly interested in the enhanced susceptibility to liver injury caused by low levels of arsenic in the setting of NAFLD. Arsenic is a natural contaminant of the drinking water and at very high concentrations has been shown to cause overt liver damage $(44,45)$. Arsenic levels found in the United States are generally considered too low to cause toxicity, however even low levels of arsenic exposure have been linked to subtle hepatic changes including capillarization of sinusoidal endothelial cells, a type of remodeling response in the liver (46). Moreover, low levels of arsenic have also been shown to sensitize the liver to inflammatory insult (47). A previous study from our group has implicated an interaction between HFD and subhepatotoxic arsenic exposure, the combination of which caused enhanced liver injury. These data support the hypothesis that HFD may sensitize the liver to otherwise innocuous toxins, including arsenic, resulting in progression of liver disease. As will be demonstrated in this work, a key effect of arsenic appears to be to enhance the deposition of fibrin ECM in response to HFD.

\section{ECM in fatty liver disease}

As mentioned above, the major part of the study of the role of the ECM in liver disease has focused on the robust increase in collagen deposition which is a hallmark of hepatic fibrosis. However, other studies have shown that other ECM components, including fibronectin, elastin, laminin and hyaluron, are also altered 
during hepatic fibrogenesis (48). Global changes in the distribution and concentration of many different ECM components likely contribute to pathological tissue remodeling and the progression of fibrogenesis.

Recent studies suggest that transitional tissue remodeling may occur before fibrosis. For example, Gillis et al showed that ethanol exposure increases the secretion of fibronectin, a structural component of the ECM, by hepatic stellate cells (HSCs) prior to their activation (28). Since activated HSCs play a role in fibrogenesis, these results suggesting that fibronectin secretion is increased prior to fibrogenesis. This group has also shown that enhanced LPS-induced fibrin accumulation caused by acute alcohol exposure is critical to liver injury and inflammation $(29,49,50)$. It is likely that there are changes in other ECM proteins contributing to hepatic inflammation. In support of this possibility, a proteomic approach used by Lai et al showed that a myriad of ECM-associated proteins are dysregulated during hepatocarcinogenesis (51) Applying this proteomics technique to changes in the hepatic ECM proteome could identify potential players in transitional tissue remodeling in early stages of fatty liver disease.

\section{Alcoholic lung phenotype}

It has been estimated that up to 190,000 people suffer from acute lung injury (ALI) annually in the United States (52). The risk of developing more severe forms of ALI such as acute respiratory distress syndrome (ARDS), is significantly higher in chronic alcoholics (53). Furthermore, a history of chronic alcohol 
consumption has been shown to increase the risk of mortality in ARDS patients (54). Subsequent rodent studies investigating the possible mechanisms underlying the detrimental effects of alcohol on the lung indicate that chronic alcohol may cause a myriad of pulmonary changes, coined the 'alcoholic lung phenotype,' including increased pro-inflammatory cytokine release (54-57), induction of oxidative stress (58), and tissue remodeling $(59,60)$. The dysregulated inflammatory response caused by alcohol is dependent on both the amount and duration of alcohol consumption and is suspected to be a key player in alcohol-related lung dysfunction (61). The exact mechanisms that result in the 'alcoholic lung phenotype' remain unclear, but, if identified, would point to potential targets for intervention in alcohol-related lung dysfunction.

\section{Parallel mechanisms of injury in the liver and lung}

Currently, the effects of chronic ethanol exposure on the liver and lung are considered to be independent of each other and thus are often studied separately. However, independent studies investigating the effects of ethanol on the liver and lung individually have revealed that similar mechanisms are at play in each organ and, more specifically, that these organs have similar responses to alcohol exposure. For example, alcohol enhances LPS-induced organ damage in both the liver and lung $(58,62)$. Additionally, oxidative stress, a well-known mediator of alcohol-induced liver injury (63) is also involved in increased susceptibility to lung injury caused by alcohol (64). Furthermore, both organs possess resident macrophages that can play a critical role in dysregulated 
inflammation after alcohol exposure (65) and both exhibit subtle changes in the composition of the extracellular matrix (ECM) following alcohol exposure. The deleterious effects of tissue remodeling are well understood with regards to hepatic (66) and pulmonary fibrosis (67), however recent work from both this lab and Roman's group have implicated more subtle ECM changes to be a key step in disease progression in the liver and lung, respectively. Specifically, the Roman lab has shown that chronic alcohol exposure increases pulmonary fibronectin deposition via alterations in both expression and degradation of ECM components (68). Similarly, our lab has shown that ethanol enhances increased hepatic fibrin deposition caused by LPS and that subsequent inflammatory damage to the liver is a direct effect of fibrin accumulation $(29,50,69-71)$.

\section{The gut-liver-lung axis}

Chronic alcohol abuse can act as a first 'hit' that leads to alterations in tissue architecture without causing detectible organ damage. This alcoholic first 'hit' can render the host susceptible to injury in the presence of a second stimulus, such as endotoxemia. Circulating levels of endotoxin (i.e. LPS) are increased in chronic alcoholics due to changes in intestinal permeability caused by alcohol exposure. This increase in circulating endotoxin appears to be a common second 'hit' that drives progression of liver and lung injury. While the role of the gut:liver axis in alcohol induced liver disease is now well-established $(72,73)$, we propose that organ-organ interactions after alcohol exposure are not limited to the gut:liver axis but may also include the lung. For example, more than 30 
years ago one cohort study showed that end-stage liver disease increased mortality associated with ARDS from $40-60 \%$ to $100 \%$ (74), suggesting that liver injury may directly impact susceptibility to lung injury. Other studies investigating the multi-organ effects of endotoxemia indicate that interaction between the liver and lung is not only likely, but may occur via LPS-induced mediators (75) including TNFa (76), an inflammatory cytokine markedly increased in both endstage liver patients (77) and experimental models of liver injury. The role of TNFa in pulmonary injury is also well established (55). As mentioned above, the effects of chronic ethanol exposure on the liver and lung have most often been studied independently. In order to study the possibility of interdependence between these two organs, a model of concomitant liver and lung injury must be established. Once established, additional studies investigating possible links between liver and lung injury will allow a better understanding of inter-organ interactions in this model.

\section{B. Aims and proposals}

\section{Protective effects of prebiotics in the setting of non-alcoholic liver disease}

Our lab has already shown that arsenic can sensitize the liver to non-alcoholic fatty liver disease caused by a high fat diet. In this model, enhanced damage correlated with an increase in hepatic inflammation and in sinusoidal fibrin deposition in the liver. Hepatic inflammation and injury caused by a HFD is mediated by multiple mechanisms, including gut dysbiosis. Indeed, HFD feeding increases gut leakiness, causing endotoxemia and systemic inflammation (78) 
and liver steatosis (79). Protecting against endotoxemia by altering the microbiota caused by HFD exposure can also protect against inflammation and metabolic syndrome in mice (80). Furthermore, the benefits of pre- and probiotics on liver injury have been established in models of alcoholic and non-

alcoholic liver disease (81-83). Enhanced liver injury caused by the combination of HFD and arsenic in this model may be mediated by interactions between HFD and arsenic on the gut microbiome. Therefore the goals of this study were to 1) determine the protective effects of oligofructose (OFC) and 2) to identify interactions between HFD, arsenic, and OFC on the gut microbiome in a model of enhanced liver injury caused by concomitant exposure to HFD and arsenic. Importantly, fibrin ECM deposition may play a key role in this response.

\section{Characterization of a model of concomitant liver and lung injury}

A number of studies independently investigating the liver and lung response to chronic alcohol exposure have indicated that similar mechanisms contribute to alcohol-induced changes in these two organs including oxidative stress, dysregulated inflammatory response, and tissue remodeling. An important function of the liver is as a host-defense system; thus liver injury and the resultant reduction in hepatic function could enhance susceptibility of other organs to injury, including the lungs. For example, mortality due to ARDS was increased to nearly $100 \%$ in individuals with end-stage liver disease (74). This as well as work by Siore et al (84) have indirectly suggested that liver injury and/or hepatic-derived mediators may drive injury in the lung. One potential link 
between liver and lung injury is the pro-inflammatory cytokine TNFa. TNFa levels in the systemic circulation are increased after alcohol exposure in both experimental models of alcohol exposure $(85,86)$ and in patients with ALD $(77)$. The resident hepatic macrophages, Kupffer cells, are known to be a major source of pro-inflammatory mediators into the systemic blood $(65,87)$ and likely contribute to the robust increase in pro-inflammatory signaling after alcohol exposure. Moreover, TNFa has also been linked to pulmonary injury in experimental models $(88,89)$. Therefore, the purpose of this study was to characterize a model of concomitant liver and lung injury and to investigate the potential role of TNFa as link between liver and lung injury after chronic alcohol exposure. These studies lay the groundwork for future studies on the interaction of the role of the liver in lung ECM remodeling in the 'alcoholic lung phenotype.'

\section{Can we characterize the hepatic extracellular proteome?}

As mentioned above, the role of the ECM in later stages of liver disease (i.e., fibrosis) is well recognized. Previous studies from this lab and other groups (28, 90) have shown that changes in the ECM also occur in early pre-fibrotic stages of liver disease. These transitional changes in the ECM appear to play a critical role in the sensitization of the liver to insult, particularly in the context of fatty

liver. While several matrix proteins have been implicated as players in liver injury and inflammation (i.e., fibrin, fibronectin) there are likely other ECM proteins that also contribute to hepatic injury. Identification of new ECM proteins that are altered by ethanol exposure would provide new insights into mechanisms of hepatic tissue remodeling that contribute to progression of ALD. There are likely 
changes in the hepatic ECM that have not yet been identified due to the insolubility of matrix proteins making them difficult to extract and analyze. Additionally, the study of alterations to the hepatic ECM has primarily been hypothesis-driven which can hamper the discovery of new potential players. The purpose of this study was to use a serial extraction technique which would allow extraction and enrichment of tightly cross-linked proteins coupled with LC-MS analysis. When paired together, these methods will allow extraction of proteins with low expression levels and poor solubility and analysis of global changes in the hepatic ECM.

\section{Overall aim of this dissertation.}

The overall aim of this dissertation is to build on the unifying hypothesis that ECM dyshomeostasis contributes to hepatic pathology, as well as to pathology in other organs, such as the lung. Indeed, as described above, the enhancement of liver injury caused by arsenic exposure in the context of HFD (Aim 1) is characterized by a robust increase in fibrin deposition; the intervention strategies that protect against this pathology will shed new insight into future studies. The development of a new model to characterize the interaction between liver and lung in the alcoholic lung phenotype (Aim 2), coupled with the new ECM proteomic strategies (Aim 3), lay the foundation for testing the hypothesis that liver injury is critical in transitional ECM remodeling that occurs with alcohol exposure (i.e., the 'alcoholic lung phenotype'). Taken together, this work will 
shed new mechanistic insight into the complex interplay between injury, matrix dyshomeostasis and disease progression. 


\section{CHAPTER II}

\section{EXPERIMENTAL PROCEDURES}

\section{A. Animals and treatments}

Mice were housed in a pathogen-free barrier facility accredited by the Association for Assessment and Accreditation of Laboratory Animal Care, and procedures were approved by the University of Louisville's Institutional Animal Care and Use Committee.

At time of sacrifice, animals were anesthetized with ketamine/xylazine (100/15 mg/kg, i.p.). Blood was collected from the vena cava just prior to sacrifice by exsanguination and citrated plasma was stored for further analysis. Prior to lavage of the lungs, $10 \mathrm{~mL}$ of PBS was perfused through the heart to flush the pulmonary vasculature of erythrocytes. Bronchioalveolar lavage (BAL) was performed by flushing the lung two times with $400 \mu \mathrm{L}$ sterile PBS. Cells in the BAL fluid (BALF) were separated by centrifugation and removed from remaining BALF and fixed on slides for further analysis (91). Portions of liver tissue and lung tissue were snap-frozen in liquid nitrogen for later analysis or fixed in $10 \%$ neutral buffered formalin for subsequent sectioning and mounting on microscope slides. A portion of liver tissue was frozen-fixed in Tissue Tek OCTCompound (Satura Finetek, Torrance, CA). Total RNA was immediately 
extracted from fresh liver and lung tissue using RNA-stat (Tel-Test, Austin, TX) and chloroform: phenol separation (see RNA isolation Methods).

\section{Arsenic, high fat diet, and oligofructose feeding}

Four week old male C57BI6/J mice were purchased from the Jackson Laboratory (Bar Harbor, ME). Food and tap water were allowed ad libitum. Mice were fed AIN-76 Purified diet (Harlan Laboratories) for one week to reduce potential confounding factors of arsenic present in standard laboratory chow (92). Mice were exposed to sodium arsenite (4.9 ppm in drinking water) or tap water for one week prior to initiating feeding with either commercially available experimental diets (Table 2.1; Harlan Laboratories, Madison WI) including a low fat diet (LFD; $13 \%$ fat in calories), a high fat diet (HFD; $42 \%$ fat in calories), or a HFD containing the prebiotic oligofructose ( $5 \% \mathrm{w} / \mathrm{w}$ OFC) for 10 weeks. This exposure level of arsenic was determined by preliminary range-finding experiments to cause no overt liver damage in mice fed low-fat diet. Food and water consumption were measured twice a week. Body weight was measured once a week

\section{Chronic model of alcohol exposure}

Eight week old male C57BL6/J mice were purchased from the Jackson Laboratory (Bar Harbor, ME) and exposed to either ethanol-containing LieberDeCarli diet (Dyets, Inc.) or iso-caloric control diet. During the exposure period, animals were housed in pairs in shoebox cages in a room held at $75^{\circ} \mathrm{F}$. Diet was provided in vacuum tubes and replaced between 4 and $5 \mathrm{pm}$ daily. Both ethanol-fed animals and their pair-fed counterparts received control diet for the 
Table 2.1. Diet Composition

\begin{tabular}{|c|c|c|c|c|}
\hline & \multicolumn{2}{|c|}{ Low Fat Diet } & \multicolumn{2}{|c|}{ High Fat Diet } \\
\hline & $\% \mathrm{kCal}$ & $\begin{array}{c}\text { Source } \\
\text { (\% by weight) }\end{array}$ & \% kCal & $\begin{array}{c}\text { Source } \\
\text { ( } \% \text { by weight) }\end{array}$ \\
\hline Protein & 19.2 & Casein & 15.2 & Casein \\
\hline Carbohydrate & 67.9 & $\begin{array}{l}\text { Sucrose/Corn } \\
\text { starch }\end{array}$ & 42.7 & $\begin{array}{l}\text { Sucrose/Corn } \\
\text { starch }\end{array}$ \\
\hline Fat & 13.0 & $\begin{array}{c}\text { Milkfat }(3.7 \%) / \\
\text { soybean oil }(1.3 \%)\end{array}$ & 42.0 & Milkfat (21.2\%) \\
\hline $\mathrm{kCal} / \mathrm{g}$ & 3.6 & & 4.5 & \\
\hline
\end{tabular}


first two days of liquid diet feeding to allow acclimation to the liquid diet feeders. After 2 days of acclimation, ethanol concentrations increased incrementally over the course of three weeks before reaching the highest ethanol concentration, $6 \%$ $(\mathrm{vol} / \mathrm{vol})$ for the final three weeks of exposure. Ethanol concentrations in the ethanol-containing diets were as follows: $0 \%$ for two days of acclimation, $1 \%$ for two days, $2 \%$ for two days, $4 \%$ for one week, $5 \%$ for one week, and $6 \%$ for three weeks. Ethanol-containing diet was provided ad libitum for the entire course of the study. Because of the relatively high caloric content of ethanol, pair-fed control animals received an iso-caloric control diet; the calories in the iso-caloric diet were matched by adding a calorie-equivalent of maltose-dextrin. To account for the reduced food consumption of ethanol-fed mice, pair-fed mice were given the volume of diet consumed by their ethanol-fed counterparts the night before. At the conclusion of the feeding period, the two diet groups were further separated into additional groups that received either LPS (E. coli; $10 \mathrm{mg} / \mathrm{kg}$ i.p Sigma, St. Louis, MO) or vehicle (saline). Animals were euthanized 4 or $24 \mathrm{~h}$ after LPS (or vehicle) injection.

\section{a. Etanercept administration}

Etanercept (Etan; Wyeth) is an FDA-approved TNFa blocker. Etanercept is a fusion protein of the human p75 TNF receptor attached to the Fc portion of human IgG1. In this study, some animals were administered etanercept (10 $\mathrm{mg} / \mathrm{kg}$ in PBS) by i.p. injection two times per week during ethanol administration, as described by others $(93,94)$. Control animals received PBS vehicle, $(1 \mu \mathrm{l} / \mathrm{g}$; i.p) twice per week. 


\section{B. Proteomics}

\section{3-step ECM extraction (95-97)}

Sample preparation and wash. Snap frozen liver tissue $(75-100 \mathrm{mg})$ was immediately added to ice-cold phosphate-buffered saline $(\mathrm{pH} 7.4)$ wash buffer containing commercially available protease and phosphatase inhibitors (Sigma Aldrich) and $25 \mathrm{mM}$ EDTA to inhibit proteinase and metalloproteinase activity, respectively. While immersed in wash buffer, liver tissue was diced into small fragments using a scalpel. The diced sample was washed 5 times to remove contaminants. Between washes, samples were pelleted by centrifugation at $10,000 \times \mathrm{g}$ for $5 \mathrm{~min}$ and wash buffer was decanted. $\mathrm{NaCl}$ extraction. Diced samples were incubated in 10 volumes of $0.5 \mathrm{M} \mathrm{NaCl}$ buffer, containing $10 \mathrm{mM}$ Tris $\mathrm{HCl}(\mathrm{pH}$ 7.5), proteinase/phosphatase inhibitors, and $25 \mathrm{mM}$ EDTA. The samples were mildly mixed on a plate shaker $(800 \mathrm{rpm})$ overnight at room temperature. The following day, the remaining tissue pieces were pelleted by centrifugation at $10,000 \times \mathrm{g}$ for $10 \mathrm{~min}$. The supernatant was saved and labeled as the $\mathrm{NaCl}$ fraction. $\underline{\mathrm{SDS} \text { extraction. }}$ The pellet from the $\mathrm{NaCl}$ extraction was subsequently incubated in 10 volumes (based on original weight) of a $1 \%$ SDS solution, containing proteinase/phosphatase inhibitors and $25 \mathrm{mM}$ EDTA. The samples were mildly mixed on a plate shaker $(800 \mathrm{rpm})$ overnight at room temperature. The following day, the remaining tissue pieces were pelleted by centrifugation at $10,000 \times \mathrm{g}$ for $10 \mathrm{~min}$. The supernatant was saved and labeled as the SDS extract. Guanidine $\mathrm{HCl}$ extraction. The pellet from the SDS extraction was incubated with 5 volumes (based on original weight) of a 
denaturing guanidine buffer containing $4 \mathrm{M}$ guanidine $\mathrm{HCl}(\mathrm{pH} 5.8), 50 \mathrm{mM}$ sodium acetate, $25 \mathrm{mM}$ EDTA, and proteinase/phosphatase inhibitors. The samples were vigorously mixed on a plate shaker at $1200 \mathrm{rpm}$ for 48 hours at room temperature; vigorous shaking is necessary at this step to aid in the mechanical disruption of ECM components. The remaining insoluble components were pelleted by centrifugation at $10,000 \times \mathrm{g}$ for 10 minutes. This insoluble pellet was retained and solubilized as described below. The supernatant was saved and labeled as the $\mathrm{GnHCl}$ fraction. Deglycosylation and solubilization. The supernatants from each extraction were desalted using ZebaSpin columns (Pierce) according to manufacturer's instructions. The desalted extracts were then mixed with 5 volumes of $100 \%$ acetone and stored at $-20^{\circ} \mathrm{C}$ overnight to precipitate proteins. The precipitated proteins were pelleted by centrifugation at $16,000 \times \mathrm{g}$ for 45 minutes. Acetone was evaporated by vacuum drying in a RotoVap for 1 hour. Dried protein pellets were resuspended in $500 \mathrm{uL}$ deglycosylation buffer $(150 \mathrm{mM} \mathrm{NaCl}, 50 \mathrm{mM}$ sodium acetate, $\mathrm{pH}$ 6.8, $10 \mathrm{mM}$ EDTA, and proteinase/phosphatase inhibitors) that contained chondroitinase $\mathrm{ABC}$ ( $P$. vulgaris; $0.025 \mathrm{U} /$ sample), endo-beta-galactosidase ( $B$. fragilis; $0.01 \mathrm{U} / \mathrm{sample}$ ) and heparitinase II ( $F$. heparinum; $0.025 \mathrm{U} /$ sample). Samples were incubated overnight at $37^{\circ} \mathrm{C}$. Samples containing the pellet remaining after the guanidine $\mathrm{HCl}$ step received $20 \mathrm{uL}$ DMSO to aide in solubilization. Protein concentrations were estimated by absorbance at $280 \mathrm{~nm}$ using BSA in deglycosylation buffer for reference standards. 


\section{Sample cleanup and preparation for liquid chromatography}

Pooled samples in deglycosylation buffer were provided to and subsequently analyzed by the University of Louisville Proteomics Biomarkers Discovery Core (PBDC). At the PBDC, samples in deglycosylation buffer were thawed to room temperature and clarified by centrifugation at $5,000 \mathrm{xg}$ at $4^{\circ} \mathrm{C}$ for $5 \mathrm{~min}$. Samples were reduced by adding $5.55 \mu \mathrm{L}$ of $1 \mathrm{M} \mathrm{DTT}$ to $50 \mu \mathrm{L}(25 \mu \mathrm{g})$ of each sample and then incubating at $60^{\circ} \mathrm{C}$ for $30 \mathrm{~min}$ after which $144.45 \mu \mathrm{L}$ of $8 \mathrm{M}$ urea in $0.1 \mathrm{M}$ Tris- $\mathrm{HCl} \mathrm{pH} 8.5$ was added to each sample. Each reduced and diluted sample was digested with a modified Filter-Aided Sample Preparation (FASP) method. Recovered material was dried in a SpeedVac and redissolved in $200 \mu \mathrm{L}$ of $2 \%(\mathrm{v} / \mathrm{v})$ acetonitrile $(\mathrm{ACN}) / 0.4 \%$ formic acid $(\mathrm{FA})$. The samples were then trap-cleaned with a C18 PROTO ${ }^{\mathrm{TM}} 300 \AA$ Ultra MicroSpin Column (The Nest Group, Southborough, MA). The sample eluates were stored at $-80^{\circ} \mathrm{C}$ for $30 \mathrm{~min}$, dried in a SpeedVac, and stored at $-80^{\circ} \mathrm{C}$ until analysis. Before liquid chromatography, dried samples were warmed to room temperature and dissolved in $2 \%(\mathrm{v} / \mathrm{v}) \mathrm{ACN} / 0.1 \%$ FA to a final concentration of $0.25 \mu \mathrm{g} / \mu \mathrm{L}$. For analysis $16 \mu \mathrm{L}(4 \mu \mathrm{g})$ of sample was injected into the Orbitrap Elite.

\section{Liquid Chromatography}

Dionex Acclaim PepMap 10075 um x 2 cm nanoViper (C18, 3 m, $100 \AA)$ trap and Dionex Acclaim PepMap RSLC $50 \mu \mathrm{M}$ x $15 \mathrm{~cm}$ nanoViper (C18, $2 \mu \mathrm{m}$, $100 \AA$ ) separating columns were used. An EASY n-LC (Thermo, Waltham, MA) UHPLC system was used with buffer $A=2 \%(v / v)$ acetonitrile $/ 0.1 \%(v / v)$ formic acid and buffer $\mathrm{B}=80 \%(\mathrm{v} / \mathrm{v})$ acetonitrile $/ 0.1 \%(\mathrm{v} / \mathrm{v})$ formic acid as mobile 
phases. Following injection of the sample onto the trap, separation was accomplished with a 140 min linear gradient from $0 \%$ B to $50 \%$ B, followed by a 30 min linear gradient from $50 \%$ B to $95 \%$ B, and lastly a 10 min wash with $95 \%$ B. A $40 \mathrm{~mm}$ stainless steel emitter (Thermo, Waltham, MA; P/N ES542) was coupled to the outlet of the separating column. A Nanospray Flex source (Thermo, Waltham, MA) was used to position the end of the emitter near the ion transfer capillary of the mass spectrometer. The ion transfer capillary temperature of the mass spectrometer was set at $225^{\circ} \mathrm{C}$, and the spray voltage was set at $1.6 \mathrm{kV}$.

\section{Mass Spectroscopy}

An Orbitrap Elite - ETD mass spectrometer (Thermo) was used to collect data from the LC eluate. An Nth Order Double Play with ETD Decision Tree method was created in Xcalibur v2.2. Scan event one of the method obtained an FTMS MS1 scan for the range $300-2000 \mathrm{~m} / \mathrm{z}$. Scan event two obtained ITMS MS2 scans on up to ten peaks that had a minimum signal threshold of 10,000 counts from scan event one. A decision tree was used to determine whether collision induced dissociation (CID) or electron transfer dissociation (ETD) activation was used. An ETD scan was triggered if any of the following held: an ion had charge state 3 and $\mathrm{m} / \mathrm{z}$ less than 650 , an ion had charge state 4 and $\mathrm{m} / \mathrm{z}$ less than 900 , an ion had charge state 5 and $\mathrm{m} / \mathrm{z}$ less than 950 , or an ion had charge state greater than 5; a CID scan was triggered in all other cases. The lock mass option was enabled ( $0 \%$ lock mass abundance) using the 371.101236 $\mathrm{m} / \mathrm{z}$ polysiloxane peak as an internal calibrant. 


\section{Data Analysis}

Proteome Discoverer v1.4.0.288 was used to analyze the data collected by the mass spectrometer. The database used in Mascot v2.4 and SequestHT searches was the 6/2/2014 version of the UniprotKB Mus musculus reference proteome canonical and isoform sequences. In order to estimate the false discovery rate, a Target Decoy PSM Validator node was included in the Proteome Discoverer workflow.

The Proteome Discoverer analysis workflow allows for extraction of MS2 scan data from the Xcalibur RAW file, separate searches of CID and ETD MS2 scans in Mascot and Sequest, and collection of the results into a single file (.msf extension). The resulting .msf files from Proteome Discoverer were loaded into Scaffold $Q+S$ v4.3.2. Scaffold was used to calculate the false discovery rate using the Peptide and Protein Prophet algorithms. The results were annotated with mouse gene ontology information from the Gene Ontology Annotations Database

\section{Clinical chemistry}

Levels of alanine aminotransferase (ALT) and aspartate aminotransferase (AST) were determined spectrophotometrically using standard kits (Thermotrace, Melbourne, Australia).

\section{Hepatic lipid measurements}

Total lipid was extracted from mouse livers as previously described $(98,99)$. Briefly, mouse liver (25-50 mg) was pulverized in a liquid-nitrogen cooled mortar and pestle. Pulverized liver tissue was incubated in a methanol:chloroform (2:1) 
mixture on ice for thirty minutes. At the end of incubation, extracts were centrifuged at $900 \times \mathrm{g}$ for $10 \mathrm{~min}$ at $15^{\circ} \mathrm{C}$. After centrifugation, lipids are partitioned into the chloroform (bottom) layer. This bottom layer was removed using a glass syringe and dried in an evaporating centrifuge. The resulting pellet was resuspended $5 \%$ fatty acid-free bovine serum albumin then frozen at $-80^{\circ} \mathrm{C}$ overnight. Prior to analysis, samples were vortexed then sonicated for 10 minutes at $37^{\circ} \mathrm{C}$ in a bath sonicator. Hepatic triglyceride (Thermo Scientific, Middletown, VA) non-esterified fatty acids (Sigma Aldrich, St. Louis MO) were determined using standard kits. Values were normalized to initial liver tissue weight.

\section{E. Histology}

\section{General morphology}

Formalin fixed, paraffin embedded liver and lung tissue were cut at $5 \mu \mathrm{m}$ and mounted on charged glass slides. Sections were deparaffinized with Citrisolv (Fisher, Waltham, MA) and rehydrated through graded ethanol. Sections were then stained with hematoxylin and eosin (H\&E). After staining, samples were dehydrated through graded alcohol, washed in Citrisolv and then mounted with Permount (Fisher, Waltham, MA).

\section{Neutrophil accumulation}

Chloroacetate esterase (CAE) staining for neutrophils was performed using the naphthol AS-D chloroacetate esterase kit (Sigma, St. Louis, MO) on paraffin embedded sections $(5 \mu \mathrm{m})$. For blinded quantitation, samples were de-identified and 10 fields were photographed at 400x magnification and saved. For liver 
tissue, the extent of CAE staining was quantified by counting the number of CAEpositive neutrophils per 1000 hepatocytes (69). For lung tissue, total CAEpositive cells were counted per 10 fields. During quantification, neutrophils were characterized by light pink (i.e. CAE-positive) staining and bi-lobed nuclear morphology.

\section{Immunohistochemistry}

Immunohistochemical staining for the marker F4/80 was performed as previously described (98). Formalin-fixed, paraffin embedded sections $(5 \mu \mathrm{m})$ were deparaffinized and rehydrated with graded ethanol solutions. Endogenous peroxidase activity was quenched by incubating slides in a $3 \% \mathrm{H}_{2} \mathrm{O}_{2}$ solution for 5 minutes at room temperature. Because the liver has high concentrations of biotin, an avidin-biotin blocking reagent (Dako, Carpinteria, CA) was used to reduce non-specific staining. Slides were then incubated in primary antibody for the F4/80 cell surface receptor (1:500 in 10\% goat serum; Abcam, Cambridge MA) for two hours at room temperature. The Vectastain Elite ABC kit was used for detection (Vector Laboratories, Inc., Burlingame, CA). Briefly, tissue was incubated in a biotinylated anti-Rat IgG secondary antibody for 15 minutes at room temperature. Tissue was then washed and incubated in a solution containing avidin-bound horseradish peroxidase (HRP) for 30 minutes. The HRP substrate 3, 3'-diaminobenzidine (DAB) was added to tissue sections until positive (brown) staining was macroscopically visible. Slides were counterstained with Hematoxylin (Dako, Carpinteria, CA) for 1 minute, washed, dehydrated through graded ethanol and then mounted with Permount (Fisher, 
Waltham, MA). Each slide contained a negative tissue section which does not receive primary antibody. The number of F4/80 macrophages in liver tissue was quantified by blinded counting of the number of $F 4 / 80$ positive macrophages per 1000 hepatocytes in $200 x$ photomicrographs.

\section{Immunofluorescence}

Immunofluorescent detection of fibrin deposition was performed as described in (69). Briefly, frozen liver sections ( $6 \mu \mathrm{m}$ thick) were fixed in $10 \%$ buffered formalin containing $2 \%$ acetic acid to solubilize all but cross-linked fibrin. Sections were blocked with PBS containing 10\% horse serum before an overnight incubation at $4^{\circ} \mathrm{C}$ with affinity purified rabbit anti-human fibrinogen IgG (Dako North America, Inc. Carpinteria, CA) diluted in blocking solution. Donkey anti-rabbit antibody conjugated to Alexa 488 (Invitrogen, Carlsbad, CA) was used as secondary antibody. Tissue was counterstained with Hoechst. Fluorescence for Alexa 488 and Hoechst was detected at excitation and emission wavelengths of $490 \mathrm{~nm}$ and $520 \mathrm{~nm}$, and $350 \mathrm{~nm}$ and $460 \mathrm{~nm}$, respectively. Immunofluorescence was visualized using a Nikon Eclipse E600 microscope (Nikon Corporation, Tokyo, Japan) and Metamorph software

\section{F. RNA isolation and real-time RT-PCR}

The hepatic mRNA expression of select genes was detected by quantitative reverse-transcriptase polymerase chain reaction (RT-PCR), which is routine for this group $(29,50,98,100)$. PCR primers and probes for TNFa, PAI-1, IL-6, and $\beta$-actin were designed using Primer 3 (Whitehead Institute for Biomedical Research, Cambridge, MA). Primers and probes for KC, MIP-2, and IL-10 were 
bought from Applied Biosystems as kits (Foster City, CA). All primers were designed to cross introns to ensure that only cDNA and not genomic DNA was amplified. Total RNA was extracted from liver tissue by a guanidinium thiocyanate-based method (RNA STAT 60 Tel-Test, Ambion, Austin, TX). RNA concentrations were determined spectrophotometrically and $1 \mu \mathrm{g}$ of total RNA was reverse transcribed using a kit (Quanta Biosciences, Gaithersburg, MD). PerfeCta qPCR Fast Mix (Quanta Biosciences, Gaithersburg, MD) was used to prepare the PCR reaction mixture. This $2 \times$ mixture is optimized for TaqMan reactions and contains $\mathrm{MgCl}_{2}$, dNTPs, and AccuFast Taq DNA Polymerase. Amplification reactions were carried out using the $A B I$ StepOne Plus machine and software (Life Technologies, Grand Island, NY) with initial holding stage $\left(95^{\circ} \mathrm{C}\right.$ for 30 seconds) and 50 cycles of a 2 -step PCR $\left(95^{\circ} \mathrm{C}\right.$ for 30 seconds, $60^{\circ} \mathrm{C}$ for 20 seconds). Fluorescent intensity of each sample was measured at each cycle to monitor amplification of the target gene. The comparative CT method was used to determine fold changes in mRNA expression compared to an endogenous reference gene ( $\beta$-actin). This method determines the amount of target gene, normalized to an endogenous reference and relative to a calibrator $\left(2^{-\Delta \Delta C t}\right)$.

\section{G. Luminex}

Cytokine levels in plasma and BALF samples were measured using the MILLIPLEX MAP Magnetic Bead Mouse Cytokine/Chemokine Panel (Millipore, Billerica, MA). The plate was read using a Luminex 100 plate reader and Exponent software. MILLIPLEX MAP assay was performed according to 
manufacturer's recommendations. Plasma (4x dilution) or BALF samples (undiluted) were added to a clear bottomed black 96 well plate. Antibodyimmobilized beads were added to samples and the plate was incubated overnight at $4^{\circ} \mathrm{C}$ on a plate shaker at $600 \mathrm{rpm}$. Detection antibody was added to wells and incubated for 1 hour followed by the addition of strepavidinphycoerythrin. Quantitative analysis of the assay was performed using the Luminex $100^{\mathrm{TM}}$ IS. Data capture and analysis were performed using the Luminex Xponent software.

\section{H. Microbiota analysis}

\section{Bacterial DNA isolation and sequencing}

DNA was extracted from frozen cecal pellet and plasma using the QIAamp DNA Stool Mini Kit (Qiagen) according to the manufacturer's directions. Briefly, cecal content (75-100 mg) was rapidly weighed, recorded, and placed into a $2 \mathrm{~mL}$ microcentrifuge tube on ice. Lysis buffer was added to each sample and the sample was vortexed until thoroughly homogenized. Homogenized sample was heated at $70^{\circ} \mathrm{C}$ for 5 minutes and then vortexed at full speed for 1 minute to pellet particles. Supernatant was removed and added to a new tube with an InhibitEX tablet (supplied by kit) which forms a matrix to pellet DNA-degrading enzymes. Samples were centrifuged to pellet inhibitors bound to the matrix; remaining supernatant was added to a new tube and incubated with proteinase K. DNA was then precipitated with ethanol and collected using the QIAmp spin column as directed by manufacturer's instructions. 
$100 \mathrm{ng}$ of cecal DNA was sent to Baylor University School of Medicine's Alkek Center for Metagenomics and Microbiome Research for $16 \mathrm{~S}$ rRNA sequencing on the 454 FLX-titanium Platform. The V3-V5 16S rRNA gene variable regions were amplified by PCR using 454 adapter-linked and barcoded primers 357F and 926R, as described previously (101). Negative controls (water) and positive controls (Staphylococcus aureus USA300 genomic DNA) were included in all steps to control for contamination. Amplicons were purified using a SPRI bead clean-up step, quantitated by picogreen assay, normalized, pooled, and then sequenced on a 454 instrument using the FLX Titanium chemistry.

\section{Bacterial DNA analysis}

The raw sequencing dataset, consisting of an average of 28,590 reads per sample with an average read length of 253 nucleotides, was deconvoluted via the PyNAST alignment tool (102) using the Greengenes reference database (August 2013 release) (103) and quality filtered with cutoff parameters of: less than 200 and greater than 1,000 bp, quality score below 30 , number of mismatches in primer greater than 0 , number of ambiguous bases greater than 6 , and maximum homopolymer runs greater than 6 . Following quality filtering, 514,622 sequences remained from the original 531,138 with an average read length of 253 nucleotides. Chimeric sequences were removed by reference and de novo chimeric filtering with UCHIME (104) utilizing the Greengenes reference database (August 2013 release) (103). Operational taxonomic unit tables based on sequences 
within the filtered data set were then generated by taxonomic binning via the Quantitative Insights Into Microbial Ecology tool (Qiime) and its various components (104). Open reference OTU picking was performed via Usearch 6.1 software (105) using $97 \%$ similarity to the clustered Greengenes database from August 2013 (106) while removing singleton OTUs from the OTU table as further quality filtering to improve specificity. The taxonomic classifications of sequence reads were identified from the phylum to the species level by the uclust based consensus taxonomy classifier (Bokulich, Rideout et al. (submitted)) with phylogenetic tree creation by Fast Tree (107). For diversity measures, an even sequence sampling depth of 16,000 sequences was used to avoid artifacts due to differing numbers of sequences per sample. PCoA analyses were performed to compare microbial community structure between samples utilizing weighted UniFrac measures (108). Linear effect size (LefSe) analyses were performed using the freely distributed Galaxy LefSe analysis module hosted at huttenhower.sph.harvard.edu/lefse/ (109). Hypothesis testing using the Dirichlet-Multinomial Distribution was performed via the HMP package for $R$ (110) within the R Statistical Computing Environment (111)

\section{Statistical Analysis}

Results are reported as means \pm standard error mean (SEM; $n=4-7$ ). ANOVA with Bonferroni's post-hoc test (for parametric data) or Mann-Whitney Rank Sum test (for nonparametric data) was used for the determination of 
statistical significance among treatment groups, as appropriate. A $p$ value less than 0.05 was selected before the study as the level of significance. 


\author{
CHAPTER III \\ OLIGOFRUCTOSE PROTECTS AGAINST ENHANCED LIVER INJURY \\ CAUSED BY ARSENIC IN A MODEL OF HIGH FAT DIET INDUCED NON- \\ ALCOHOLIC STEATOHEPATITIS - ROLE OF THE ECM
}

\title{
A. Introduction
}

Exposure to high concentrations of arsenic (As) is associated with a myriad of health effects including skin lesions, hypertension, cardiovascular disease, respiratory disease, and malignancies of the skin and internal organs (112). The liver, a major site of arsenic metabolism, is a known target of arsenic toxicity (113). Indeed, chronic arsenic exposure causes hepatomegaly, non-cirrhotic portal fibrosis and portal hypertension (113-115). The concentrations of arsenic found in the US water supply are generally considered lower than those necessary to cause overt liver damage, however lower levels of arsenic can cause more subtle changes in the liver (46) and can sensitize the liver to injury by another insult (47). Therefore, while arsenic levels in the US may not be high enough to directly injure the liver, there is evidence that low levels of arsenic may interact with other risk-modifying factors to cause hepatic injury.

Obesity is another major health concern for the US and globally. More than one third of American adults are obese (116), and it is expected that the number 
of obese individuals in the US will double by 2050 (117). Obesity is associated with a myriad of health effects including insulin resistance, diabetes, and nonalcoholic fatty liver disease (NAFLD). NAFLD is a spectrum of disease states including simple steatosis, non-alcoholic steatohepatitis, and fibrosis and cirrhosis. Studies have shown that about $20-30 \%$ the general population has NAFLD (118) however studies have shown that the prevalence of NAFLD can more than double in obese cohorts (119). Despite the high incidence of NALFD in obese populations, only a minority of obese individuals ever progress to more severe stages of liver disease, such as non-alcoholic steatohepatitis. Risk factors for primary NAFLD include obesity, type II diabetes and dyslipidemia (120). However it is likely that other unidentified risk factors contribute to progression to more severe stages of liver disease (i.e. NASH).

Interestingly, there is potential overlap in areas of risk for arsenic exposure and obesity. For example, rural communities, which have both artesian water supplies that are unregulated by Clean Water Act, often have high arsenic concentrations; these areas also generally have a high prevalence of obesity. In a study investigating the possible interaction between obesity and arsenic, this group demonstrated that low levels of arsenic synergistically enhance liver damage caused by high fat diet (98). These data suggest that underlying risk factors, such as obesity, may modify the risk of hepatic injury caused by subhepatotoxic levels of arsenic exposure. However, the mechanism(s) underlying enhanced liver injury remain unknown. 
The role of the gut:liver axis in liver disease is well recognized. Indeed, liver injury is associated with dysbiosis, disruption of gut barrier integrity, and leakage of inflammatory bacterial-derived components (i.e., endotoxin) from the gut into the systemic circulation. Obesity can affect gut permeability by decreasing the barrier function of the epithelium (78). Additionally, both obesity and arsenic have been shown to alter gut flora (121). Interestingly, probiotics, live bacteria cultures which promote commensal bacteria growth, can preserve intestinal barrier integrity and protect against liver injury in animals $(81,122)$. Clinical studies have shown that probiotic supplementation improves patient outcome compared to standard therapy (123) via gut-mediated mechanisms. Prebiotics, dietary compounds that favor the growth and maintenance of commensal bacteria, may also confer protective effects in the setting of liver injury via gutmediated mechanisms. For example, Cani et al showed that the prebiotic oligofructose (OFC) can protect against inflammation and endotoxemia by regulating the microbiota of animals fed high fat diet (78). Recently, arsenic exposure has been shown to also cause dysbiosis in mice (124), which could, in principle, exacerbate the effect of HFD. The purpose of the current study was to test this hypothesis and to determine if the prebiotic oligofructose can protect against enhanced liver injury caused by arsenic in a mouse model of NAFLD. 


\section{B. EXPERIMENTAL PROCEDURES}

\section{Animals and treatments}

Animals were exposed to combinations of HFD, arsenic and the prebiotic oligofructose as described in Chapter II.

\section{Clinical Chemistry}

Plasma levels of ALT and AST were determined as described in Chapter II

\section{Lipid determination}

Lipids were extracted from snap-frozen liver as described in Chapter II.

Triglycerides and non-esterified fatty acids were determined as described in Chapter II.

\section{Immunostaining}

Hematoxylin and eosin staining, immunohistochemical staining for $F 4 / 80$ and immunofluorescent staining for fibrin were performed on liver tissue as described in Chapter II.

\section{RNA isolation and real-time PCR}

Details for RNA isolation and real time PCR are described in Chapter II.

\section{Cecal DNA extraction}

DNA was extracted from cecal content as described in Chapter II.

\section{Microbiome analysis}

Metagenomic analysis of the cecal microbiome was performed as described in Chapter II.

\section{Statistical analysis}

Statistical analysis was performed as described in Chapter II. 


\section{C. $\underline{\text { Results }}$}

\section{Body and organ weight}

All animals gained weight and there was no mortality or morbidity in any group during the course of the study. As observed previously (98), arsenic did not significantly affect food consumption or body mass gain in animals fed either LFD or HFD (Figure 3.1A and B). High fat diet significantly increased food consumption $\sim 20 \%$ and body mass gain $\sim 50 \%$ compared to low fat diet alone (Figure 3.1C). Whereas OFC supplementation did not affect food consumption, it did significantly attenuate the increase body mass gain caused by HFD; this effect of OFC was independent of the presence of arsenic in the diet.

\section{OFC protects against liver Injury}

HFD significantly increased the percent liver to body weight compared to low fat diet (Figure 3.2B). Liver weight to body weight percent was not significantly affected by concomitant arsenic exposure or by oligofructose feeding. As expected by the hepatomegaly observed, 10 weeks of HFD feeding dramatically increased lipid accumulation in the liver (Figure 3.2A); this pathologic change comprised both macrovesicular and microvesicular steatosis. Changes in quantity of hepatic triglycerides and non-esterifieid fatty acids (NEFA) (Figure 3.2B) were also determined. HFD alone increased hepatic triglyceride and NEFA content compared to LFD alone by more than 4 fold. Arsenic administration did 


\section{Figure 3.1: Model parameters}

(A) Animals were exposed to arsenic (or tap) water for 11 weeks. One week after the start of arsenic exposure experimental diet (e.g. LFD, HFD, or HFD +OFC) was provided as described in Chapter II. (B) Body weight and food consumption were recorded once per week for the duration of the experiment. Data are

shown as $+/$ - SEM $(n=6-10)$. ${ }^{a}, \mathrm{p}<0.05$ compared to tap water control; ${ }^{\mathrm{b}}, \mathrm{p}<$ 0.05 compared to LFD; ${ }^{c}, p<0.05$ compared to no OFC. 
A

Begin Begin

Arsenic Diet

Sacrifice
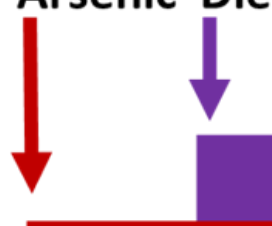

\section{0 weeks Experimental Diet}

11 weeks 5 ppm As Water

B

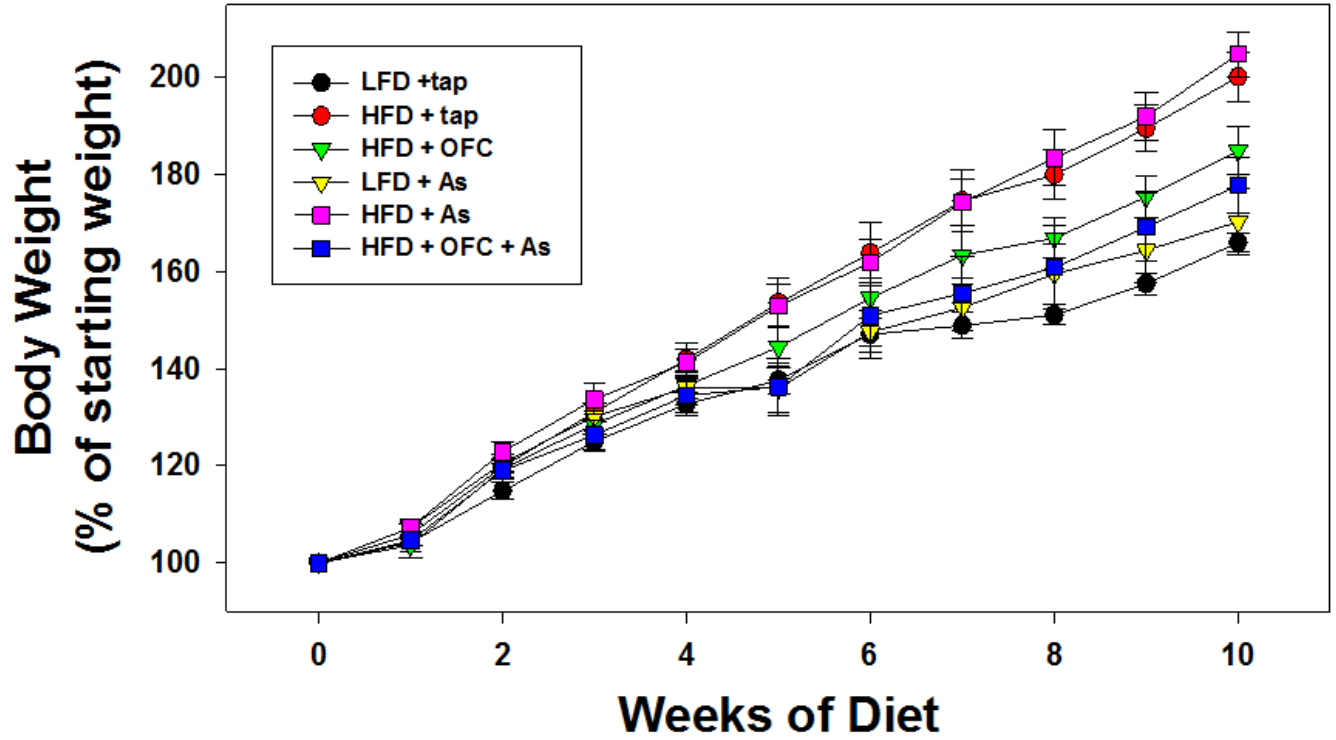

C
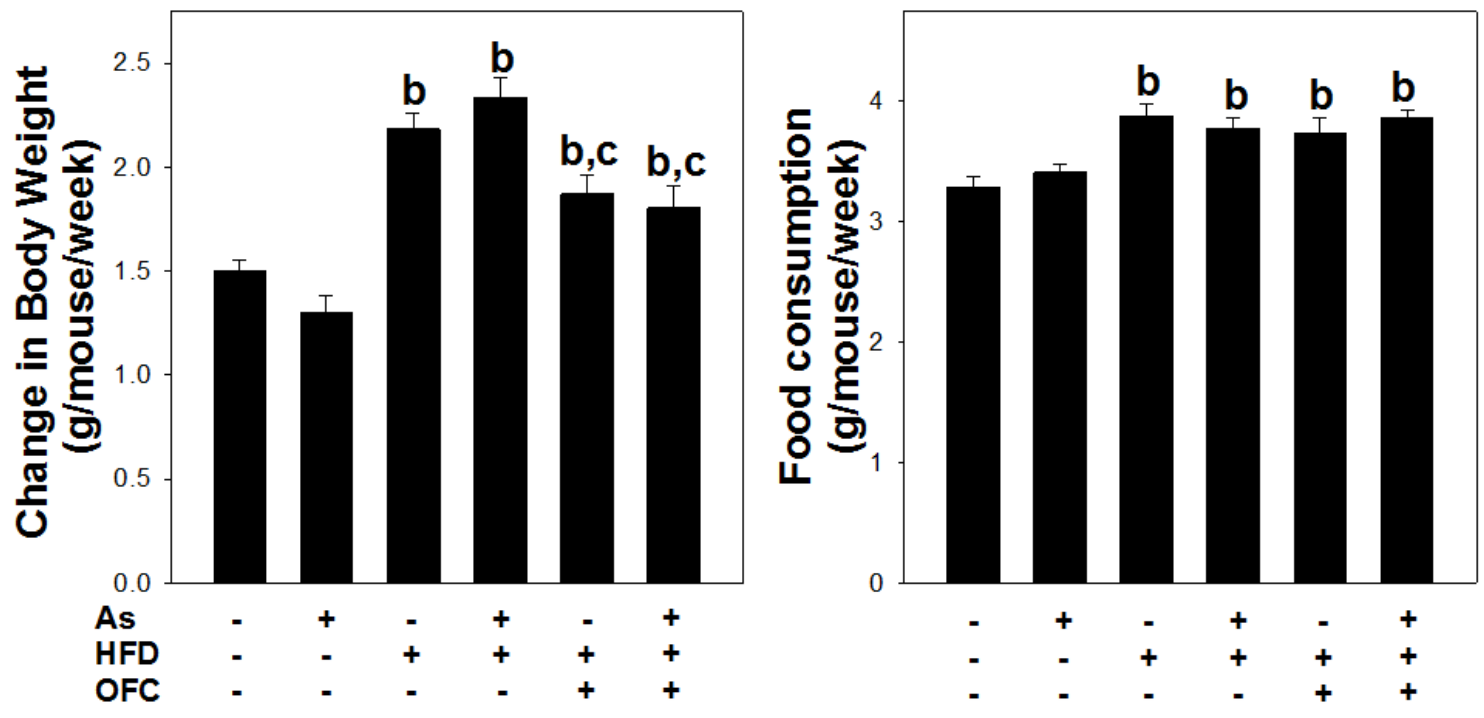


\section{Figure 3.2: OFC protects against liver injury and steatosis.}

(A) Representative photomicrographs depicting hematoxylin \& eosin staining (H\&E, 200x; top left) are shown (B) Liver to body weight percent (LW/BW\%) was determined as described in Materials and Methods. Hepatic non-esterified fatty acid (NEFA) and triglyceride (TG) content (top right) were determined using kits as described in Materials and Methods. Plasma levels of alanine and aspartate aminotransferase were determined as an index of liver injury. Quantitative data are shown as +/- SEM $(n=6-10) .{ }^{a}, p<0.05$ compared to tap water control; ${ }^{b}, p$ $<0.05$ compared to LFD; ${ }^{c}, p<0.05$ compared to no OFC. 
A

Tap
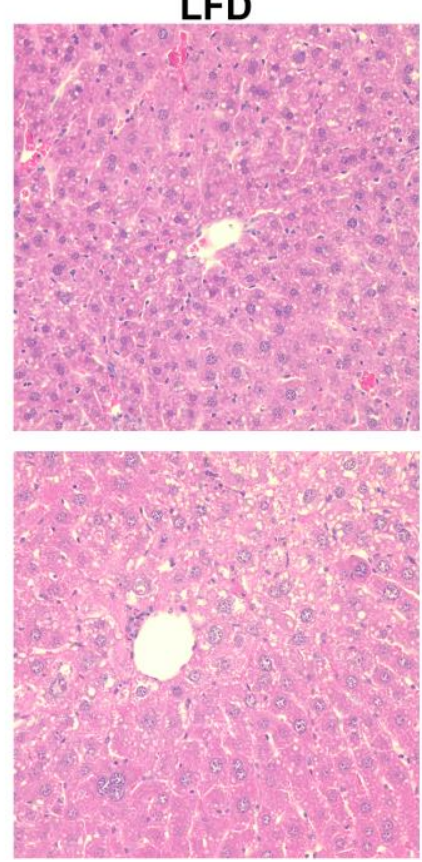

HFD
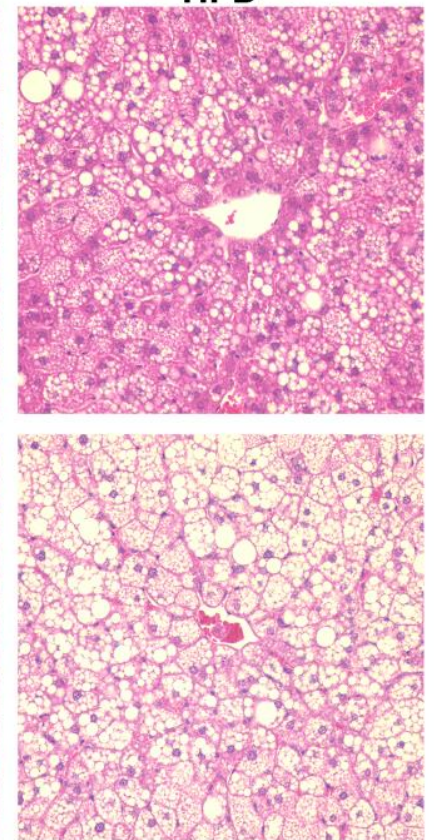

HFD + OFC
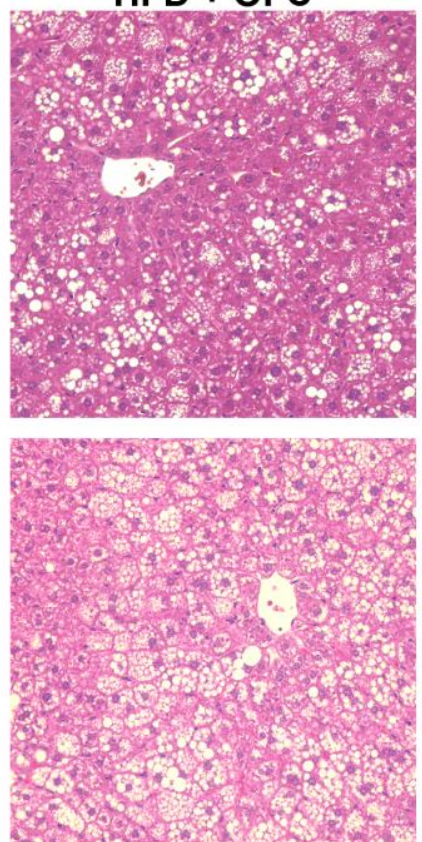

B

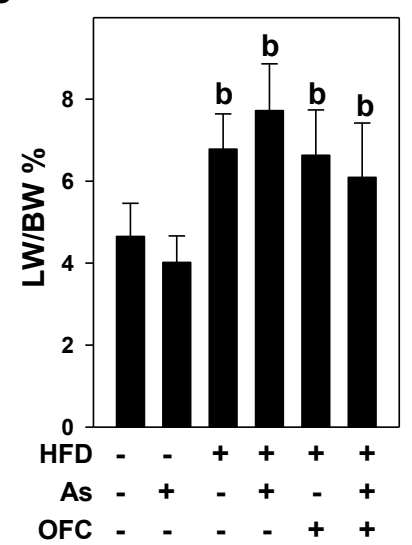

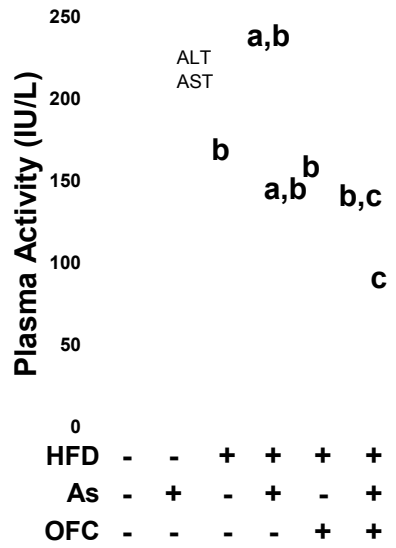


not affect steatosis or hepatic triglyceride or NEFA content when, in either LFDor HFD-fed animals. Oligofructose administration blunted fat accumulation and significantly decreased triglyceride content compared to animals that received either HFD or arsenic + HFD without OFC. Interestingly, OFC had no significant effect on NEFA content of the liver.

HFD feeding also increased the appearance of necroinflammatory foci in the liver (Figure 3.2A). Circulating levels of the liver enzymes alanine aminotransferase (ALT) and aspartate aminotransferase (AST) were determined as indices of liver damage (Figure 3.2B). HFD alone significantly increased ALT levels, but did not significantly affect AST levels. Whereas arsenic did not significantly affect plasma levels of either ALT or AST in the LFD group, it significantly enhanced the increase in these variables caused by HFD under these conditions by $\sim 50 \%$. The addition of OFC to animals receiving HFD did not affect the transaminase levels in the absence of arsenic. However, OFC administration completely blunted the increase in both ALT and AST caused by the combined exposure of arsenic and HFD.

\section{OFC protects against inflammation}

Immunohistochemistry for F4/80, a marker of activated macrophages, was performed as an index of inflammation (Figure 3.3A). Neither arsenic nor HFD alone altered the number of $\mathrm{F} 4 / 80$ positive macrophages; however the combination of arsenic + HFD significantly increased the number of hepatic 
Figure 3.3: OFC protects against hepatic inflammation.

(A) Representative photomicrographs of immunohistochemical staining for F4/80 (200x) are shown. (B) Number of F4/80 positive cells were counted per 1000 hepatoctyes as described in Chapter II. C. Real time RT-PCR was performed for the pro-inflammatory geneTNF $\alpha$ as described in Chapter II. Data are shown as +/- SEM $(n=6-10) .{ }^{a}, \mathrm{p}<0.05$ compared to tap water control; ${ }^{\mathrm{b}}, \mathrm{p}<0.05$ compared to LFD; ${ }^{c}, p<0.05$ compared to no OFC. 
A

LFD

HFD

HFD + OFC

Tap
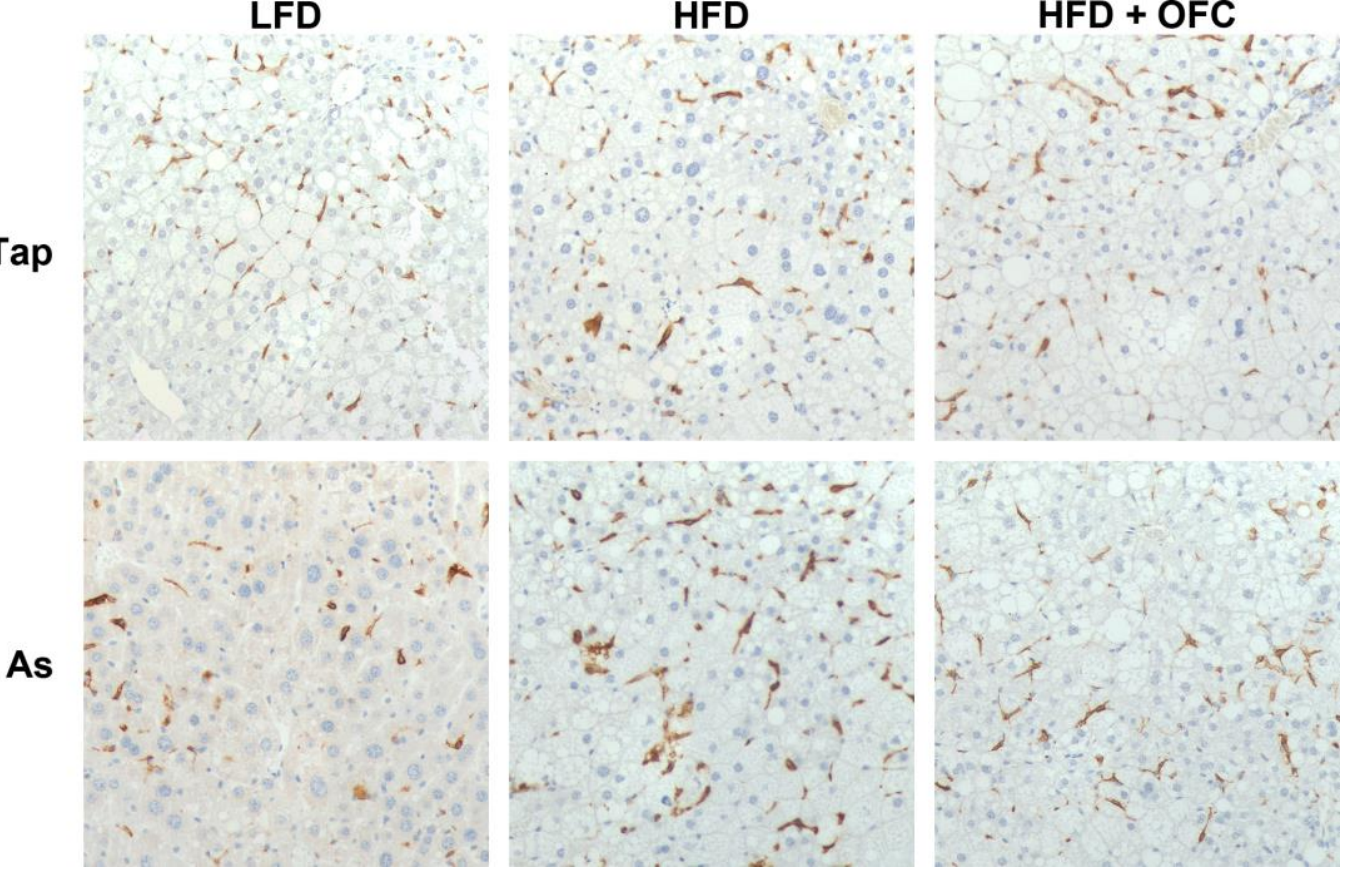

B
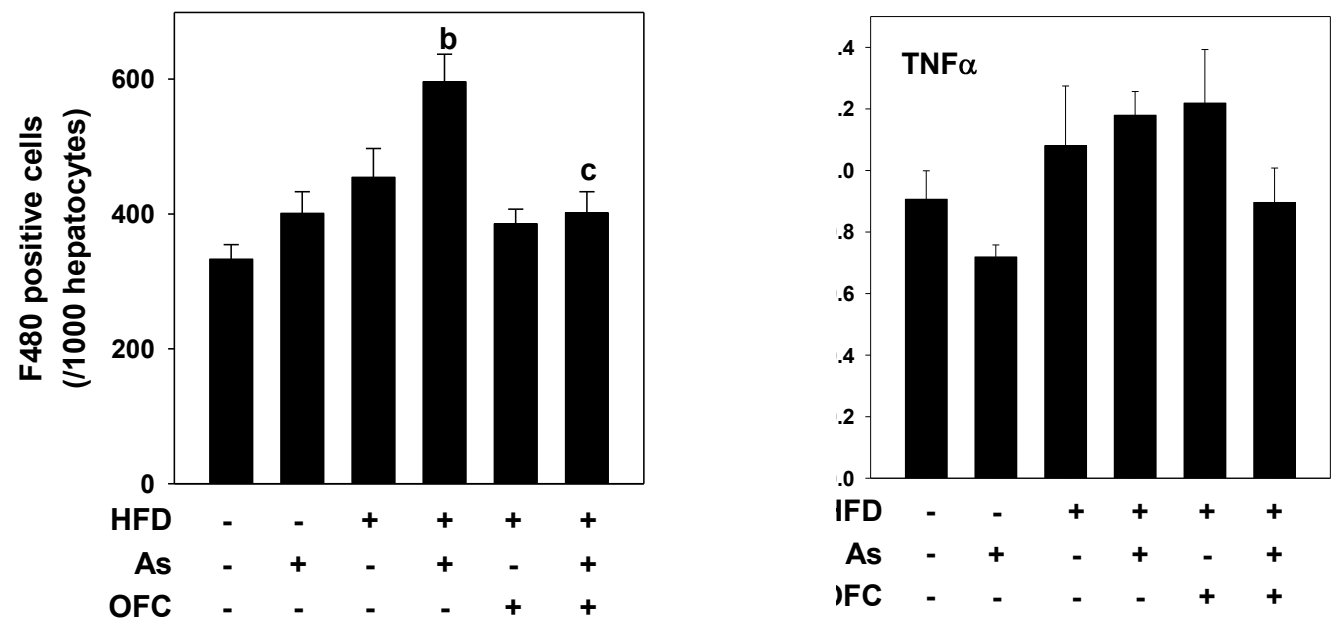
macrophages compared to arsenic or HFD alone, as was observed previously (98). The administration of OFC significantly blunted the increase in number of activated macrophages caused by the combination of arsenic and HFD and values were similar to those of LFD controls.

\section{OFC protects against fibrin deposition}

Previously, the enhancement of HFD-induced liver injury caused by arsenic correlated with a significant increase in hepatic expression of PAl-1 (98). PAl-1 is a major inhibitor of both tissue-type plasminogen activator (tPA) and urokinase-type plasminogen activator (UPA), and is therefore a key inhibitor of fibrin degradation (i.e., fibrinolysis) by plasmin. Fibrin deposition was determined by immunofluorescent staining and quantitation of fluorescent signal. LFD-fed animals given either tap or arsenic-containing water had minimal fibrin deposition in the liver (Figure 3.4A). HFD alone increased deposition of fibrin by $\sim 2$-fold. As observed previously (98), the combination of HFD + arsenic significantly increased the expression of PAI-1 and fibrin deposition in liver (Figure 3.4B). OFC administration almost completely prevented the changes caused by arsenic under these conditions.

\section{Effects on the microbiome}

The effects of arsenic, HFD, and OFC on relative abundance and total abundance of bacteria was determined at the phylum level (Figure 3.5A). As expected, the most abundance bacteria phyla were Bacteroidetes and 


\section{Figure 3.4: OFC protects against sinusoidal fibrin accumulation.}
A. Representative photomicrographs (400x) of immunofluorescent staining for fibrin are shown. B. Fibrin immunofluorescence (green color) was quantitated using Metamorph software. C. Hepatic PAI-1 mRNA expression was determined using real time RT-PCR. Data are shown as +/- SEM $(n=6-10) .{ }^{a}, \mathrm{p}$ $<0.05$ compared to tap water control; ${ }^{b}, p<0.05$ compared to LFD; ${ }^{c}, p<0.05$ compared to no OFC. 
A

Tap

As
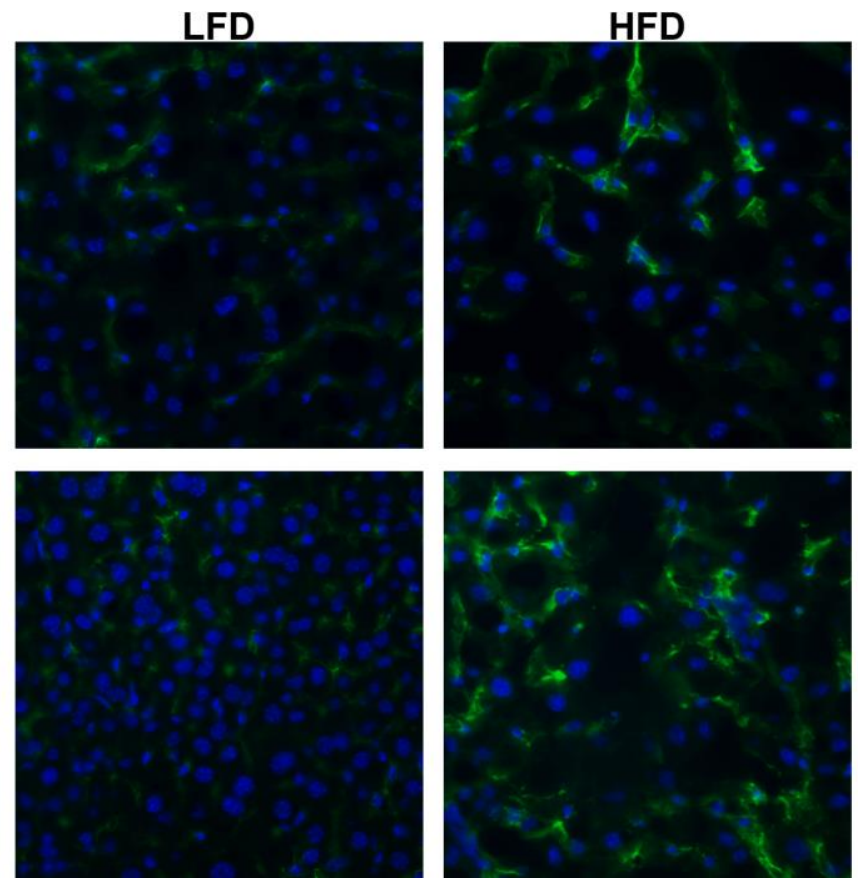

B

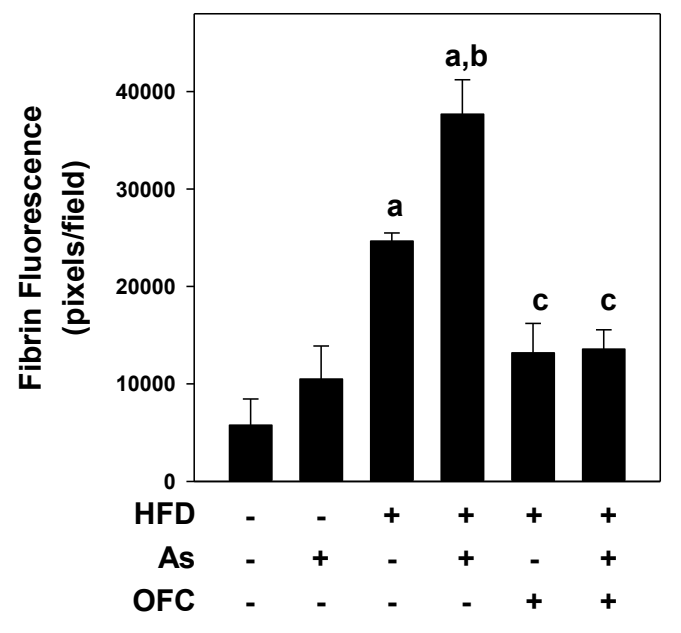

HFD + OFC
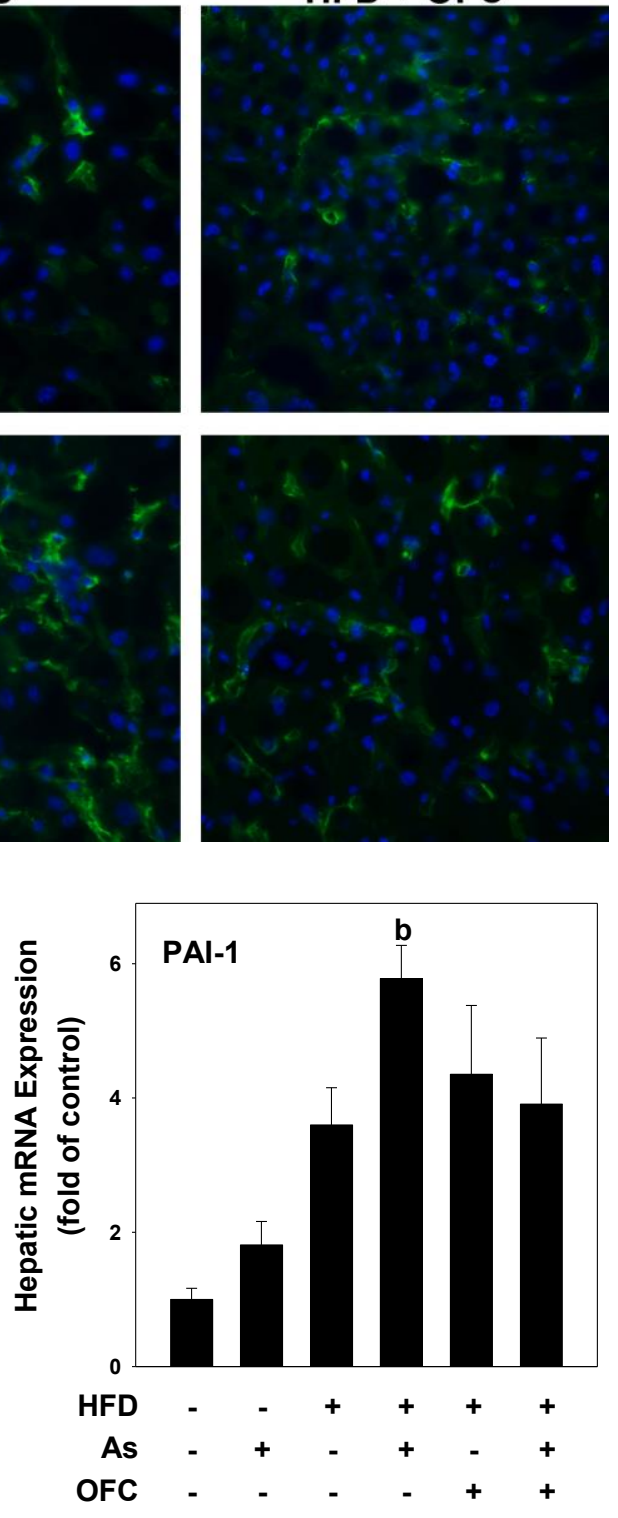
Firmicutes. Arsenic exposure alone caused a loss of the Verrucobiorobia in animals that were fed a LFD. Interestingly, this effect was lost in animals that were fed a HFD; however, the combination of arsenic and HFD did lead to an increase in the gram-negative phyla Bacteroidetes. Oligofructose feeding increased the relative abundance of Bacteroidetes with a concomitant decrease in Firmicutes compared to all other groups that did not receive the prebiotic. Additionally, OFC restored the growth of Tenericutes which was lost in animals that received a HFD. OFC also resulted in a robust increase in total bacteria compared to animals that did not receive OFC.

a diversity (Figure 3.5B) was used as a measure of diversity within a sample. Interestingly, the greatest $\alpha$ diversity was observed in animals exposed to a LFD. HFD feeding reduced $\alpha$ diversity and the combination of HFD and OFC resulted in the lowest $\alpha$ diversity of all six groups. $\beta$ diversity was used as a measure of diversity between samples (Figure 3.5C). As expected, samples that represent a single treatment group clustered more closely together, suggested a higher degree of similarity among the samples.

Linear discriminant analysis effect size (LefSe) was used to identify the enrichment of bacteria within treatment groups. When all six treatment groups were compared, unique enrichment profiles were observed for each group (Figure 3.6A). In a LefSe comparison between the LFD and LFD + arsenic, enrichment was primarily seen in animals that received tap water, suggesting that arsenic exposure resulted in decreases in these bacteria including the 
Figure 3.5: Effects of HFD, arsenic, and OFC on bacterial abundance and diversity.

A. The top stacked bar chart shows the relative abundance of bacteria phyla for each group. Absolute abundance (bottom) was determined by averaging the total number of OTUs found in each bacterial phyla per group. B. Alpha diversity, a measure of unique OTUs present in a given sample, is shown as an index of diversity within a sample. Alpha rarefaction curves were determined by counting the number of sequences found in a sampling of bacterial DNA. During the first sampling, all sequences identified were counted towards the number of observed OTUs; for each additional sampling, only unique OTUs are counted and added to the number of observed OTUs for that sample. This process is repeated until a total of 16,000 sequences are sampled. C. Beta diversity is shown as a measure of diversity between samples. This measure was determined using principle coordinate analysis of the phylogenetic diversity between samples and the differences in OTU abundance between samples. Samples that are closer in proximity have microbiomes that are more similar in term of phylogenetic diversity and OTU abundance. 
A

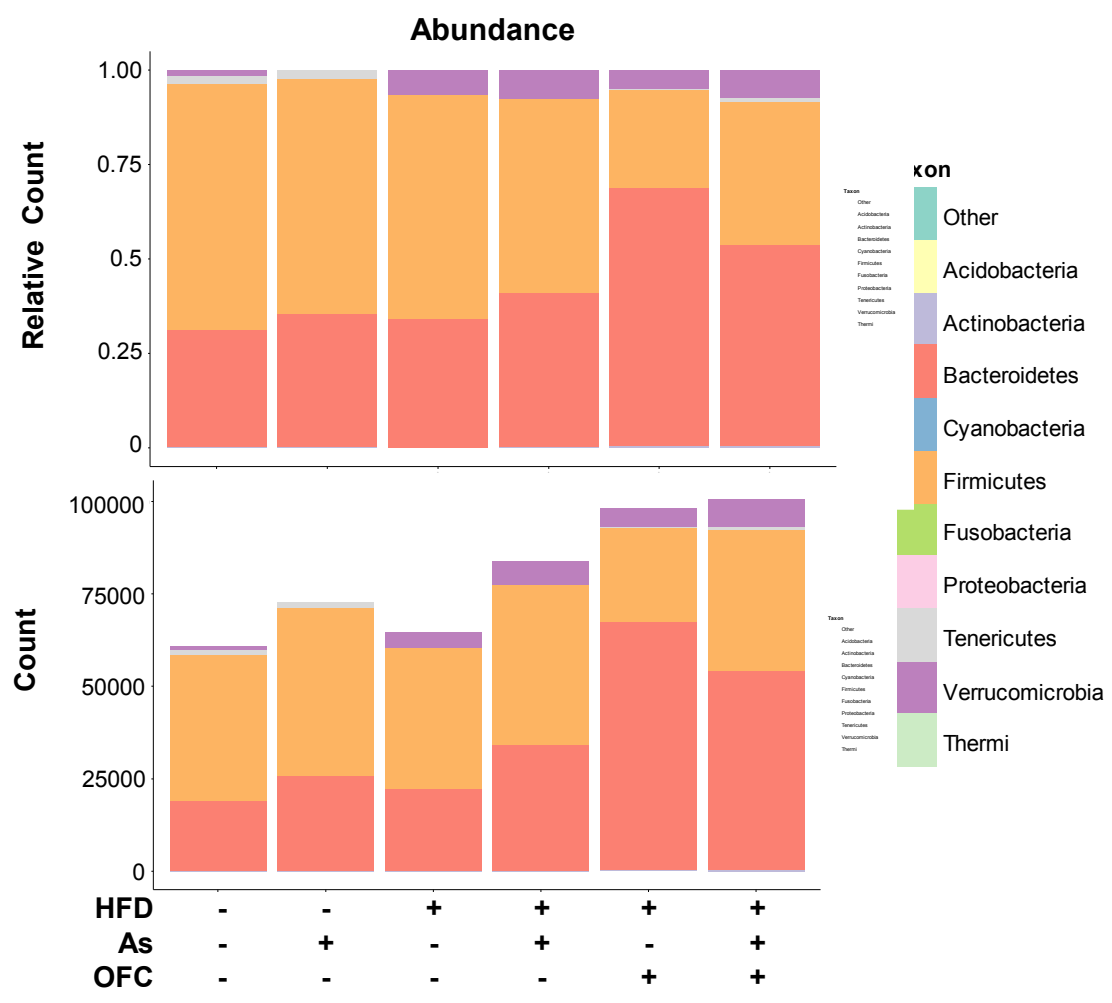

B

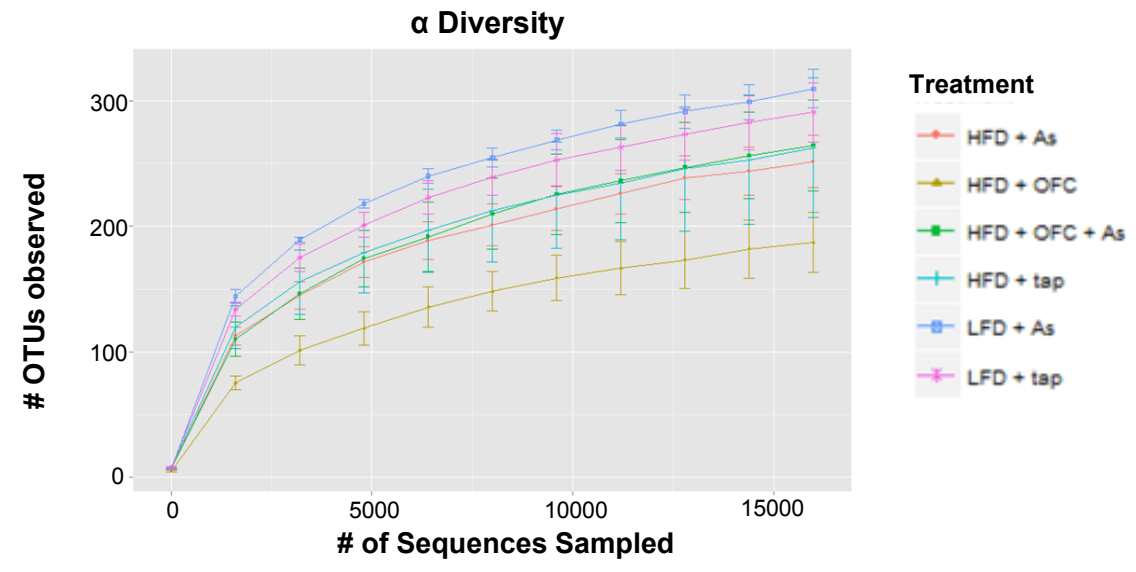

C

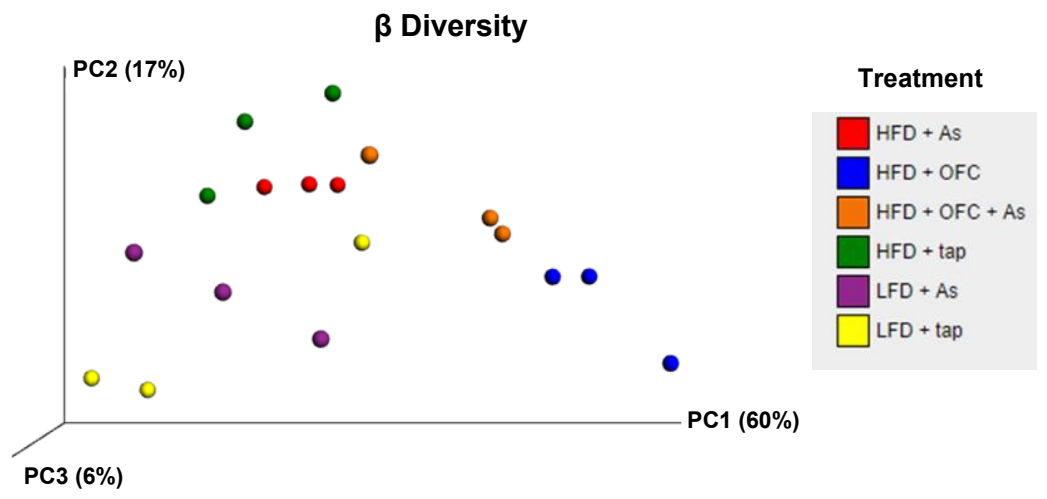


Figure 3.6: HFD, arsenic, and OFC exposure caused unique changes to the microbiome.

LefSe analysis was used to determined enrichment of taxa between groups. Effect size is determined using linear discriminant analysis (LDA). A. LefSe analysis of all six treatment groups is plotted on a cladogram. Each treatment group is represented by a unique color; highlighted taxa indicate enrichment of that taxa within the treatment group. Effect size is represented by the size of the circular intersection points between the taxa. B. Bar plot of LefSe analysis comparing the gut microbiomes of animals fed either LFD or a LFD and arsenic (top). Each bar represents a bacterial taxa that was enriched by LFD. The length of the bar represents the effect size. C. Bar plot of LefSe analysis comparing the gut microbiomes of animals fed either HFD (blue), HFD and arsenic (red), or HFD, arsenic, and OFC (green). The length of the bar represents effect size. 
A

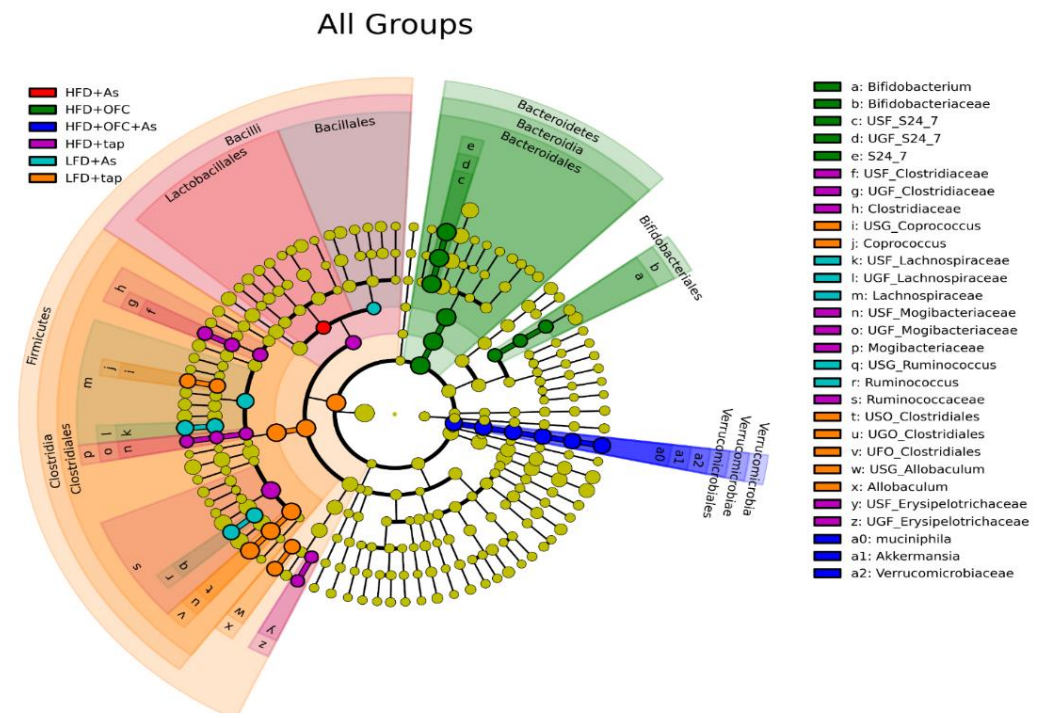

B

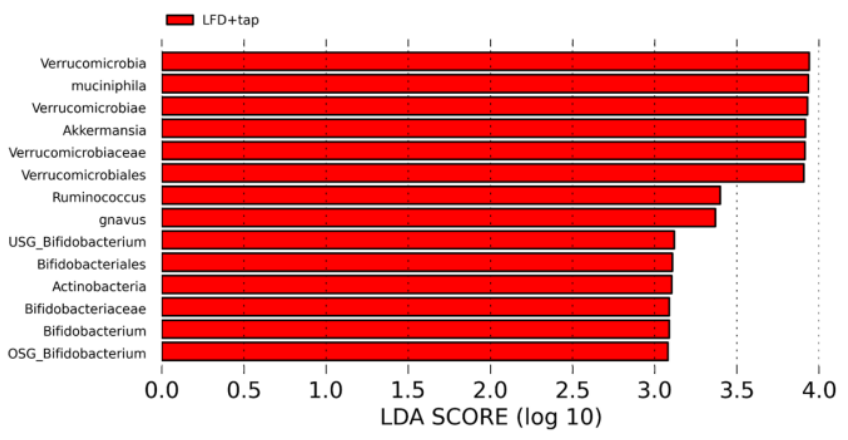

C

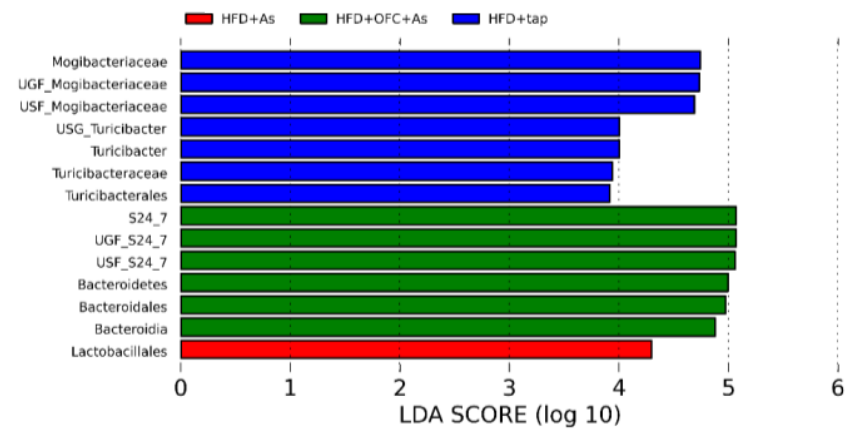


commensal Bifidobacterium (Figure 3.6B). Comparisons between animals fed HFD with or without arsenic and OFC showed that arsenic exposure was associated with a decrease in Mogibacteriacaceae and Turicibacter (Figure $3.6 C)$.

\section{DISCUSSION}

The GI tract is home to approximately 100 trillion gut microbes (125). The microbes residing in the gut regulate many important physiological functions including metabolic processing, energy production, and immune system development (125). Therefore, it is not surprising that dysbiosis of the gut flora is associated with a myriad of disease states locally in the GI tract including diarrhea/malabsorption $(126,127)$ and irritable bowel disease $(128,129)$. Because of the critical importance of the microbiome in immune surveillance and tolerance, changes in the gut microbiome are also linked to autoimmune and diseases (130-132). The gut microbiome does not only contribute to diseases of the GI tract. Indeed, several studies indicated that distal organ systems, including the CNS, are negatively affected by gut dysbiosis (133).

A major mechanism by which gut dysbiosis may contribute to systemic disease is via changing/impairing the $\mathrm{Gl}$ tract barrier function. For example, rodent models of obesity have been associated with altered gut microbiota that favor gut leakiness $(78,79)$ and endotoxemia $(78,80)$. Furthermore, dysbiosis associated with obesity contributes to the development of inflammation $(78,80)$ metabolic syndrome $(134,135)$, and non-alcoholic fatty liver injury (79). The 
understanding that the $\mathrm{GI}$ tract contributes to liver injury (i.e., the gut-liver axis) has been understood for over a century and was first shown experimentally more than 60 years ago by Rutenberg et al, when they demonstrated that nonabsorbable antibiotics protected against diet-induced liver injury (136). Since then, many studies using a myriad of models including antibiotics $(137,138)$, and germ-free mouse models (139) have provided additional evidence implicating the microbiome in the susceptibility and development of liver disease. Today, the gut-liver axis and enteric dysbiosis, including that caused by a HFD, is a generally accepted component in the development of liver disease.

Although the role of arsenic in altering the microbiome is less well studied, arsenic is known to alter the microbial components of soil (140). A recent study by Lu et al has shown that high levels of arsenic can cause dysbiosis in mice (124). In their study, metagenomic analysis of the gut flora of mice exposed to $50 \mathrm{ppm}$ arsenic showed that arsenic significantly altered the abundance of some microbial families and also resulted in functional changes in the microbiome's metabolic profile (124). The changes in the gut flora caused by arsenic in the current study were more subtle than those seen by Lu and colleagues; this difference may be attributed, in part, to the $>10$-fold lower arsenic concentrations used in the current study. Nevertheless, arsenic exposure did change the mouse microbiome in the current study; importantly, arsenic further exacerbated the dysbiosis caused by HFD feeding in mice. Importantly, supplementation with oligofructose, a prebiotic that has been demonstrated to protect against dysbiosis and favor commensal bacteria, almost completely prevented the enhancement of 
HFD-induced liver injury caused by arsenic. These results therefore support the hypothesis that arsenic mediates liver injury under these conditions via mechanisms that involve the gut-liver axis.

There are several potential mechanisms by which the microbiome may mediate arsenic-induced liver injury. First, the changes in the microbiome caused by diet (HFD and OFC) may affect arsenic metabolism and absorption. Indeed, interactions between gut microbes and arsenic are bidirectional. For instance, Pinyayev et al showed that bacteria from the mouse cecum can metabolize arsenic (141) and others have shown that the human gut flora can metabolize arsenic into toxic metabolites $(142,143)$. Whereas this is distinctly possible, it should be noted that concentrations that are 10-fold higher than used here were not directly hepatotoxic (47). Alternatively, the effects of arsenic on the microbiome may mediate the observed hepatotoxicity. Indeed, arsenic exposure expanded the population of Gram negative bacteria that could produce endotoxins, which are well known for contributing to fatty liver injury. However, whether the effects are driven by microbiome changes on arsenic levels, or by arsenic changes on the microbiome, these changes may potentiate arsenic toxicity locally on the gut epithelium (144) and contribute to enhanced arsenic toxicity distally in the liver.

In addition to direct effects on the microbiome, the protective effect of OFC feeding under these conditions could also be 'upstream' of arsenic. Specifically, OFC feeding significantly attenuated the increase in hepatic lipids caused by HFD, both in the presence and absence of arsenic. The multi-hit hypothesis of 
fatty liver disease involves not only an increase in gut-derived products that damage the liver, but also an increased sensitivity to the damage caused by these gut-derived products. Indeed, fatty livers are more sensitive to a second toxic 'hit,' including gut-derived LPS $(145,146)$. Therefore, the favorable effects of OFC on hepatic steatosis may indirectly protect against the injury caused by HFD and/or arsenic by blunting this sensitization. In support of this hypothesis, enhanced liver injury caused by arsenic + HFD co-exposure was not due to an increase in steatosis per se, but rather due to an enhanced hepatic inflammatory response. Therefore, OFC may also be protecting against damage in this model by preventing hepatic sensitization caused by HFD-induced endotoxemia. Such a mechanism is supported by previous studies that arsenic-exposed livers are more sensitive to exogenous LPS (47) and that liver injury here in OFC-fed mice was insensitive to arsenic administration.

In conclusion, protection against enhanced liver injury conferred by OFC is likely due to both direct effects on the microbiome, as well indirect effects on hepatic sensitization. These findings are in-line with previous work, in which OFC supplementation was shown to protect against direct effects of HFD on the gut microbiome and barrier function (80). Prebiotics favor an environment in which commensal bacteria can thrive, in lieu of direct delivery of commensal bacteria (i.e., probiotics). As such, this approach may yield more predictable and stable results. Nevertheless, these results serve proof-of-principle that protecting against microbiome changes caused by arsenic can prevent the enhancement of inflammatory liver injury caused by this environmental contaminant. 


\section{CHAPTER IV \\ CHARACTERIZATION OF THE LINK BETWEEN LIVER AND LUNG INJURY \\ IN THE SETTING OF ALCOHOL EXPOSURE - THE ROLE OF TNFa}

\section{A. Introduction}

Alcohol use is widespread in the United States with more than half of Americans reporting alcohol consumption on a weekly basis (147). It has long been known that chronic ethanol consumption can lead to development of a myriad of health effects spanning a variety of organs including the brain, gut, liver and lungs. Indeed, the effects associated with chronic alcohol consumption have made alcohol the third leading risk factor globally for disease and disability (148) at a cost of more than $\$ 166$ billion annually to treat the medical consequences of alcohol abuse (33). Despite the known health risks, alcohol consumption remains pervasive in today's culture. In toto, alcohol consumption is responsible for $\sim 6 \%$ of all disability-adjusted life years (DALY) lost in the United States (149), most of which are attributable to alcohol-induced toxicity.

The liver is well recognized as a major target of alcohol-induced organ injury. This is due in part to the high concentrations of alcohol reached in the portal blood as well as the detrimental effects of ethanol metabolism (e.g., oxidative stress). Alcoholic liver disease (ALD) is actually a spectrum of disease states that include simple steatosis (fat accumulation), steatohepatitis, and in more 
severe cases fibrosis and cirrhosis. A major focus in ALD therapy is to treat the decompensation associated with end-stage ALD (i.e., cirrhosis). Indeed, the sequelae of a failing liver (e.g., ascites, portal hypertension, and hepatorenal syndrome) are generally what causes death in end-stage ALD. Although the successful treatment of these secondary effects prolongs the life of ALD patients, this therapy is only palliative; there are currently no therapies to halt or prevent the progression of ALD.

Alcohol consumption can also contribute to lung damage. In contrast to the liver, alcohol consumption does not appear to directly cause any overt pulmonary pathology; however, alcohol is known to exacerbate the severity of lung damage from other causes. It has been estimated that up to 190,000 people suffer from acute lung injury (ALI) annually in the United States (52). The risk of developing more severe forms of ALI such as acute respiratory distress syndrome (ARDS) is significantly higher in people who meet the diagnostic criteria for alcohol use disorders (53). Whereas alcohol consumption does not cause overt pathology in the lung, studies have shown that experimental alcohol exposure can lead to subtle changes to this organ, which has been coined the 'alcoholic lung phenotype.' This effect of alcohol is characterized by dysregulated inflammatory response $(55,56)$, oxidative stress $(58,150)$, and tissue remodeling $(59,60)$. The dysregulated inflammatory response caused by alcohol in the lung is dependent on both the amount and duration of alcohol consumption and is suspected to be a key player in alcohol-related lung dysfunction (61). The exact mechanisms that mediate the development of the alcoholic lung phenotype and 
increased risk of ALI/ARDS remain unclear. Better understanding of this complex process could identify potential therapeutic targets to treat or prevent alcohol-related lung dysfunction, such as ALI/ARDS.

It is now clear that many human diseases are a multi-stage, multi-hit process; it is therefore not surprising that multiple cells within a target organ contribute to disease pathology. Indeed, system level analyses of disease, and organ-organ interactions, are again gaining the attention of the research community. Several studies indicate interdependence between liver and lung in organ pathology. For example, mortality in ALI in the setting of severe hepatic disease is almost $100 \%$ (151). In experimental models of liver injury, vascular permeability and leukocyte activation in lungs are increased (152). Studies investigating the multi-organ effects of endotoxemia indicate that interaction between the liver and lung are not only likely, but may occur via alcohol-induced mediators (75) including TNFa (76), an inflammatory cytokine markedly increased in ALD (77).

The purpose of this study was to characterize a model of concomitant liver and lung injury in the setting of chronic alcohol exposure. It was hypothesized that chronic alcohol exposure acts as a first 'hit' that sensitizes these organs to a second inflammatory stimulus (e.g., bacterial lipopolysaccharide; LPS). The parallel mechanisms of liver and lung injury in this '2-hit' model were explored. Furthermore, the potential interdependence of liver and lung injury via systemic release of TNFa was also determined. 


\section{B. Experimental procedures}

\section{Animals and treatments}

Animals were exposed to ethanol and/or LPS as described in Chapter II.

Some animals also received the TNFa blocker etanercept as described in Chapter II.

\section{Histology}

Liver and lung tissue was stained with hematoxylin \& eosin and chloroacetate esterase as described in Chapter II.

\section{Clinical Chemistry}

Serum levels of alanine aminotransferase (ALT) and aspartate aminostransferase (AST) were determined using standard kits as described in Chapter II.

\section{RNA isolation and real-time RT-PCR}

RNA was isolated from liver and lung tissue as described in Chapter II. Real time RT-PCR was performed for the genes TNFa, IL-6, IL-10, KC, MIP-2, and the endogenous control $\beta$-actin as described in Chapter II.

\section{Luminex}

Luminex technology was used to determine the levels of TNFa, IL-10, MIP-2, and $\mathrm{KC}$ in the plasma and BAL as described in Chapter II

\section{Statistical analysis}

Data were analyzed as described in Chapter II. 


\section{Results}

Throughout the duration of dietary exposure, all mice gained $\sim 0.6 \mathrm{~g} / \mathrm{week}$, with no differences between dietary and treatment groups. In saline-treated mice fed control diet, liver weights (as percent of body weight) were $4.7 \pm 0.1 \mathrm{~g}$, whereas ethanol-fed mice had liver weights of $4.8 \pm 0.2 \mathrm{~g}$. Liver weights in both groups were unchanged by LPS exposure.

\section{Ethanol-induced steatosis enhanced LPS-induced liver injury}

As expected, 6 wks exposure to EtOH-containing diet caused mild liver damage, characterized predominantly by microvesicular fat accumulation in midzonal regions of the liver (Figure 4.1A; H\&E staining). LPS administration to pair-fed animals increased the accumulation of inflammatory foci in liver. Feeding $\mathrm{EtOH}$-containing diet increased the severity and frequency of necroinflammatory foci caused by LPS administration. Plasma levels of alanine

aminotransferase (ALT) and aspartate aminotransferase (AST) were determined as indices of liver injury (Figure 4.1B). Chronic ethanol exposure significantly increased ALT and AST levels in the plasma. LPS alone did no significantly affected circulating levels of ALT or AST. The combination of ethanol exposure and LPS significantly increased plasma ALT and AST 4 hours after LPS administration; 24 hours after LPS administration, AST values remained significantly elevated compared to controls 
Figure 4.1: Effects of chronic ethanol pre-exposure on LPS induced liver injury and inflammation.

(A) Representative photomicrographs of liver tissue after hematoxylin \& eosin staining (200x; H\&E) and chloroacetate-esterase staining (400x; CAE). (B) Plasma levels of alanine-aminotransferase (ALT; black bars) and aspartateaminotransferase (AST; grey bars) were used as an index of liver injury. (C) Quantitation of hepatic neutrophil content. Data are shown as $+/$ - SEM ${ }^{a}, p<$ 0.05 compared to pair fed controls, ${ }^{b} p<0.05$ compared to LPS alone. 
A

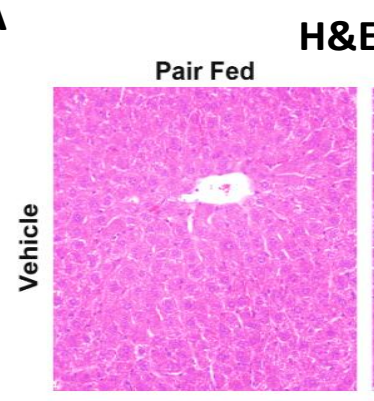

H\&E

EtOH Fed
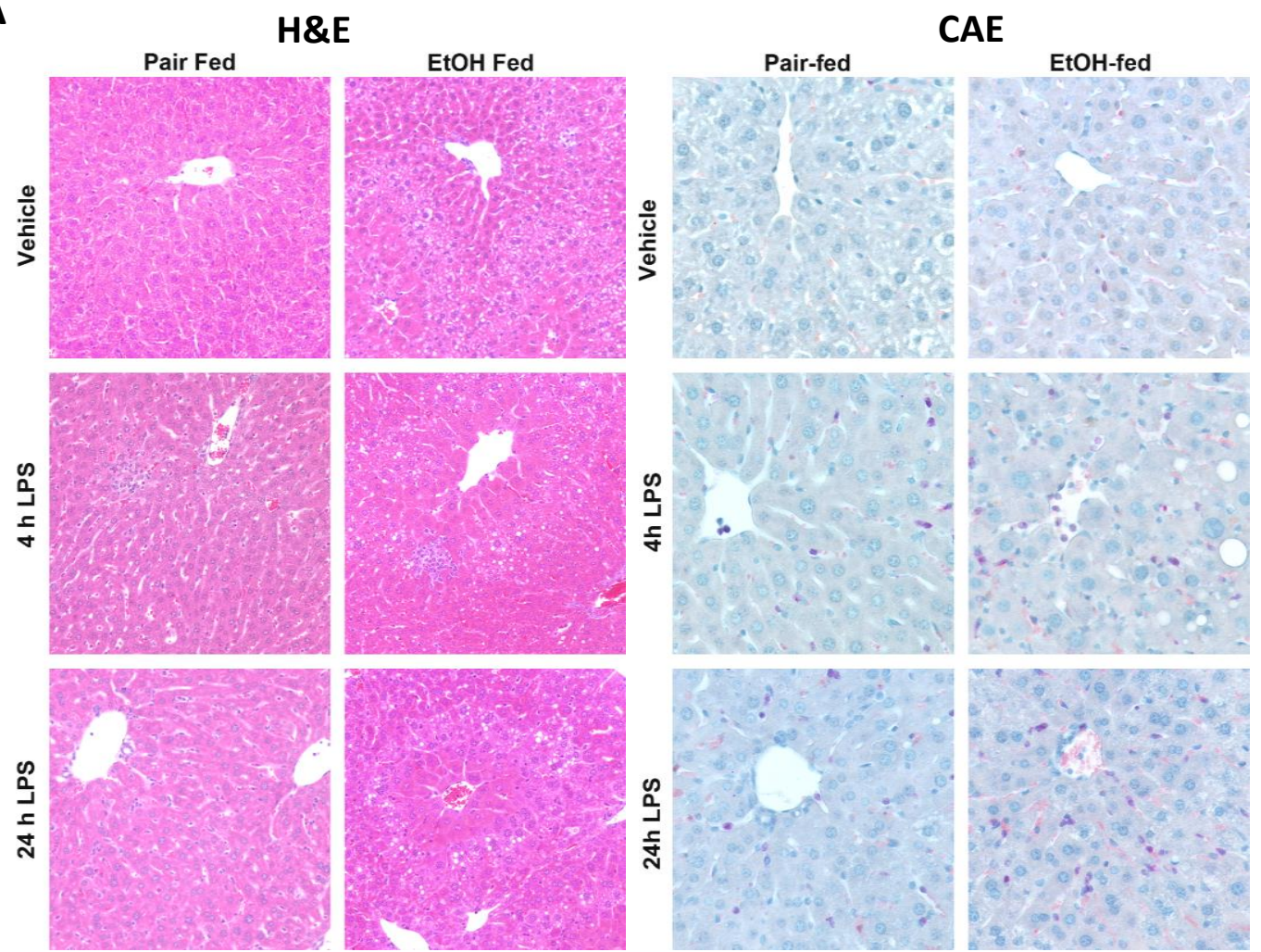

B
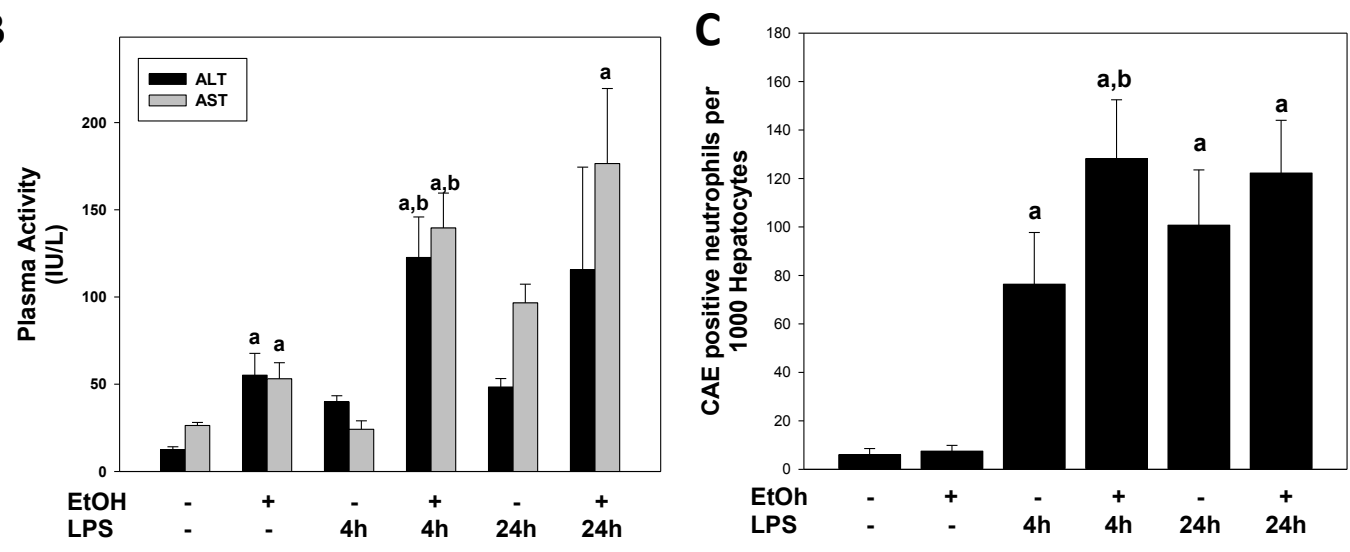
In addition to hematoxylin and eosin staining, Figure 4.1 also shows staining for neutrophils using chloroacetate esterase staining (CAE staining). Chronic ethanol exposure did not significantly affect neutrophil infiltration compared to their pair-fed counterparts (Figure 4.1A, right panels). As was expected, LPS increased the number of neutrophils in the liver compared to vehicle controls. Ethanol pre-exposure dramatically enhanced neutrophil infiltration caused by LPS.

\section{Ethanol enhanced LPS-induced lung injury and inflammation}

In liver, LPS injection caused noticeable pathologic changes as early as $4 \mathrm{~h}$ after administration (Figure 4.1A). Pathology in the lung tissue was also changed as early as $4 \mathrm{~h}$ as evidenced by inflammatory damage, however alveolar hemorrhage was not observed until the 24 hour timepoint (Figure 4.2). At this timepoint, LPS increased areas of alveolar hemorrhage in the lung, which can be observed in $\mathrm{H}+\mathrm{E}$ staining, as indicated by an increase in the number of red blood cells in the pulmonary interstitium (Figure 4.2A, left panels). Hemorrhage was macro-heterogeneous, with some areas exhibiting a higher degree of hemorrhaging than others. Analogous to findings in liver, EtOH enhanced LPSinduced lung damage, as characterized by an increase in the frequency and severity of alveolar hemorrhage caused by LPS. The increase in alveolar wall thickness caused by LPS also appeared to be more pronounced after ethanol exposure. CAE staining of the lung tissue showed a significant increase in neutrophils in the lung tissue $4 \mathrm{~h}$ after LPS administration. Chronic ethanol 
Figure 4.2: Effects of chronic ethanol pre-exposure on LPS induced lung injury and inflammation.

(A) Representative photomicrographs of lung tissue after hematoxylin \& eosin staining (400x; H\&E) and chloroacetate-esterase staining (400x; CAE). (B) Cell number in the bronchioalveolar lavage fluid (BALF). (C) Quantitation of pulmonary neutrophil content. Data are shown as $+/-$ SEM ${ }^{a}, p<0.05$ compared to pair fed controls, ${ }^{b} p<0.05$ compared to LPS alone. 
A

H\&E
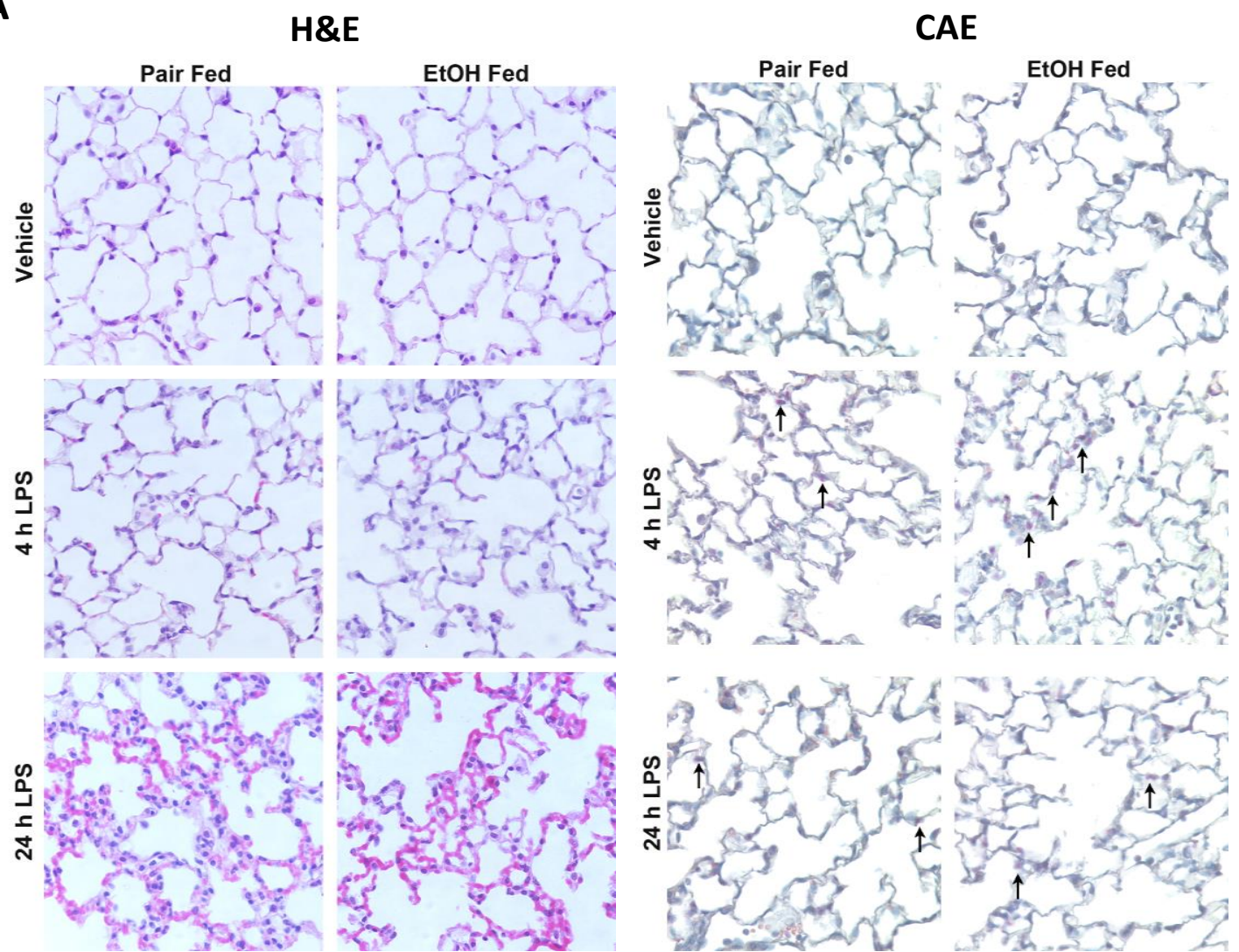

B

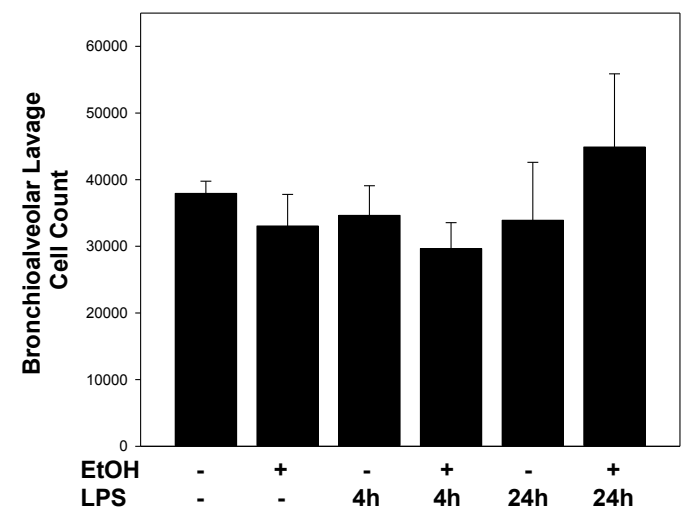

C

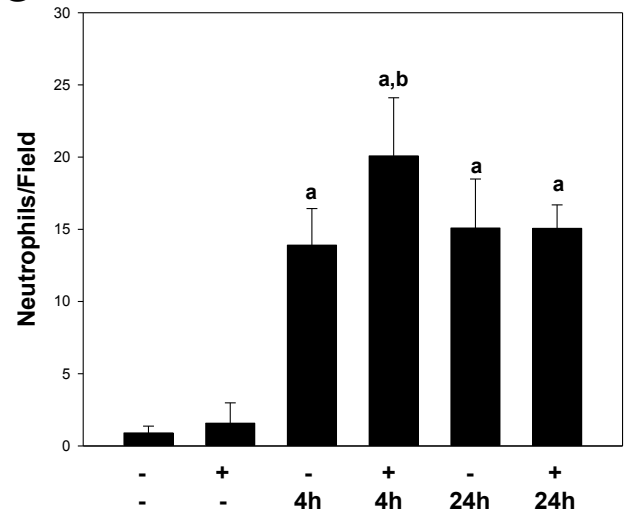


exposure enhanced the effect of LPS at the 4 hour timepoint. Incidentally, 24 hours after LPS administration, neutrophil infiltration persisted, but was no longer significantly different between ethanol and pair-fed groups.

\section{Ethanol differentially alters cytokine/chemokine expression in the liver and lung}

The pathologic assessment of liver (Figure 4.1) and lung (Figure 4.2) indicates that chronic ethanol exposure enhanced the inflammation and damage caused by LPS in both organs caused by LPS. To further characterize the mechanisms by which ethanol causes these effects, the mRNA expression of key inflammatory mediators (TNFa, IL-6, IL-10, PAI-1, MIP-2, and KC) were determined via real time RT-PCR (Figure 4.3A). Chronic alcohol exposure alone caused no significant changes in mRNA expression of these select cytokines in either the liver or lung. However, LPS administration alone significantly increased hepatic mRNA expression of TNFa, IL-6, IL-10, PAI-1, and MIP-2, with PAI-1 and MIP-2 being the most robustly induced; LPS did not significantly affect the hepatic mRNA expression of KC. As expected, chronic ethanol exposure enhanced the increase in TNF $\alpha$ and IL-6 mRNA expression levels seen with LPS alone in the liver.

Similar to the results seen in the liver, LPS administration alone increased the pulmonary mRNA expression of all mediators; however, the increase in TNFa was much less pronounced compared to liver and MIP-2 was the inflammatory mediator that was most robustly altered. In contrast to the liver, chronic ethanol 
Figure 4.3: Effect of chronic ethanol exposure on LPS-induced cytokine expression.

(A) Cytokine mRNA expression in the liver and lung at the four-hour LPS time point. (B) Inflammatory cytokine levels in the plasma and bronchoalveolar fluid (BALF) at the four-hour time point. Data are shown as +/- SEM ${ }^{a}, p<0.05$ compared to pair fed controls, ${ }^{b} p<0.05$ compared to LPS alone. 
A
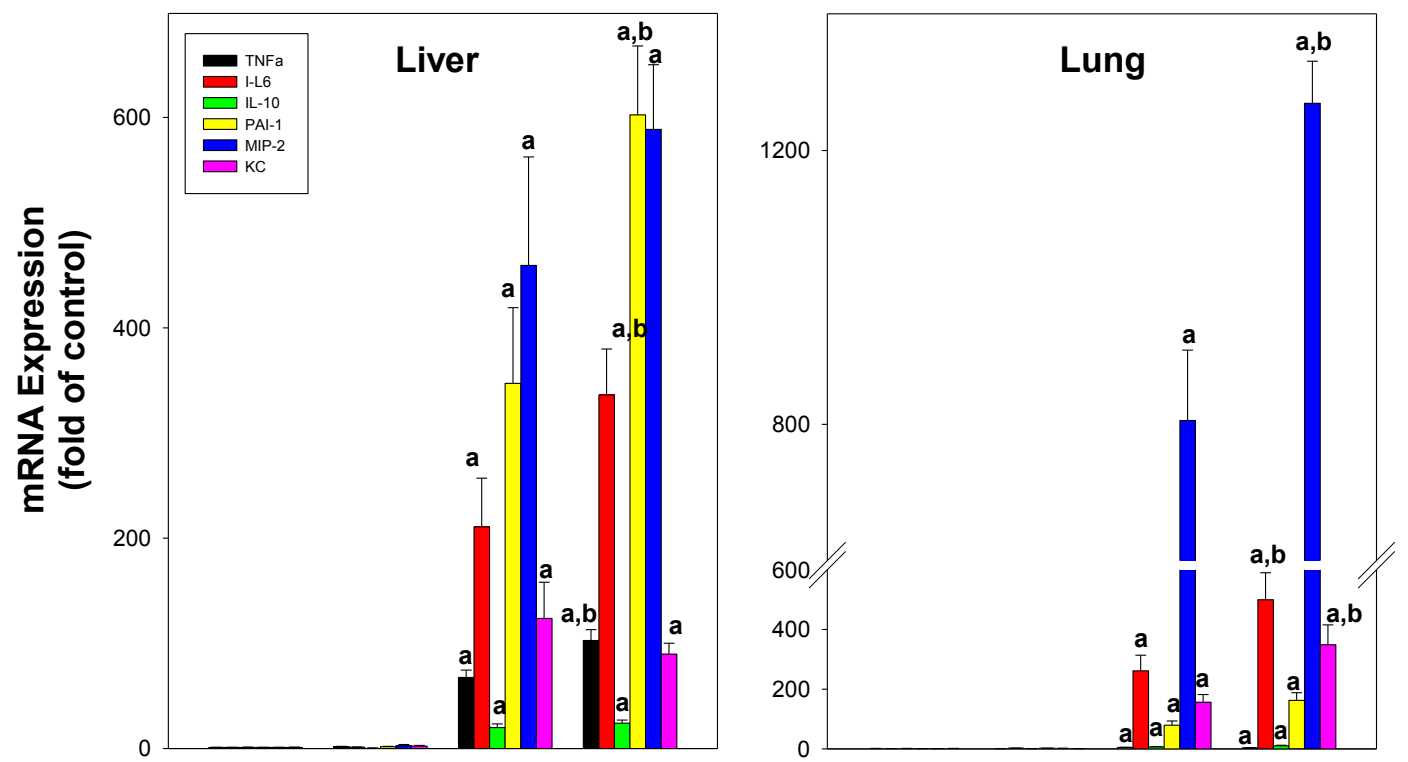

B
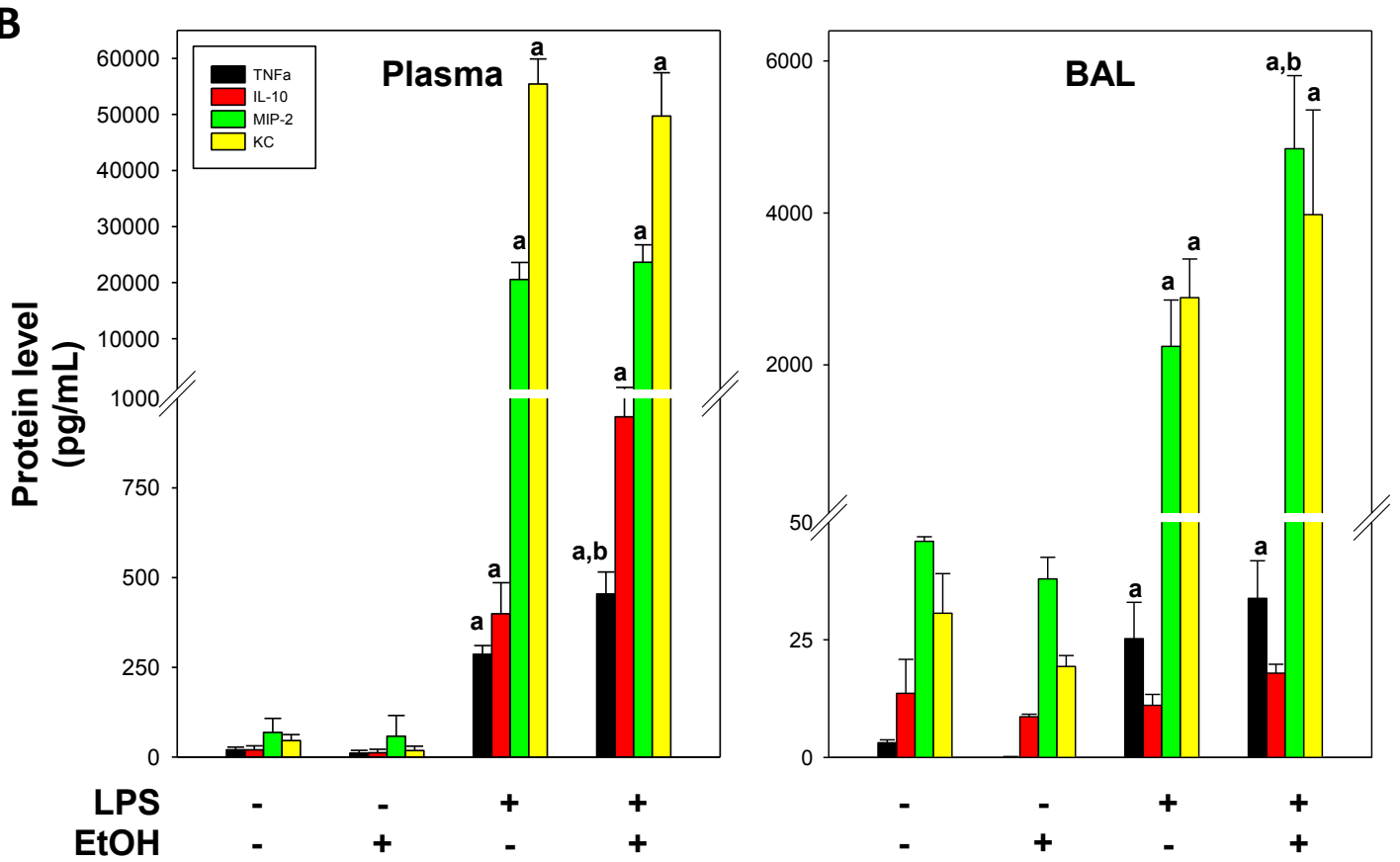
exposure did not significantly enhance the increase in TNFa expression seen with LPS alone. However, ethanol did enhance the increases in IL-6, PAI-1, and MIP-2 mRNA caused by LPS in the lung, but the pattern of that enhancement differed from liver tissue. Indeed the increase in MIP-2 caused by EtOH + LPS in the lung was $>2$-fold higher than observed in the liver. Additionally, ethanol exposure significantly enhanced the increase in expression of $\mathrm{KC}$ in the lung, an effect not observed in liver tissue.

The liver is the major contributor to the increase in inflammatory mediators in plasma in response to i.p. LPS administration (153). Likewise, BALF contains inflammatory mediators produced by lung tissue. The presence of the select mediators (TNFa, IL-10, MIP-2 and KC) in the plasma and BALF were therefore determined via Luminex assay (Figure 4.3B). With the exception of IL-10 in the BALF, LPS treatment increased the levels of all four inflammatory mediators in the plasma and BALF. In plasma, chronic ethanol exposure enhanced the increase in systemic IL-10 and TNF $\alpha$ caused by LPS, but did not significantly affect MIP-2 and KC. In contrast, TNFa and IL-10 levels in the BALF were both insensitive to ethanol preexposure, but the increase in MIP-2 and KC caused by LPS administration was dramatically enhanced.

\section{Effect of etanercept on indices of injury and inflammation.}

Previous studies have established that TNFa production is critical for subsequent pulmonary chemokine (e.g., MIP-2 and KC) production and injury in experimental models of ARDS (154). It is unclear, however, whether the source 
of TNFa needs to be pulmonary or systemic in order to see this effect in the lungs (155). Therefore, etanercept was used to deplete systemic TNFa, then measured the effects of etanercept on indices of damage and inflammation in the liver and lung. As seen in Figure 4, co-administration of etanercept during the course of the study partially protected against liver injury, as determined by AST levels (Figure 4.4B), but did not affect steatosis or inflammation caused by ethanol or LPS (Figure 4.4A), respectively. Etanercept administration decreased levels of freely soluble TNFa in response to the combination of LPS in the plasma and BALF, by $33 \%$ and $60 \%$, respectively. Interestingly, etanercept administration caused a 1.3-fold decrease in plasma MIP-2 levels which correlated with a decrease in MIP-2 mRNA expression in the liver. Etanercept also caused decreased MIP-2 in the BALF as well as a 3.2-fold decrease in KC levels in the BALF. The decrease in BALF KC levels correlated with a 3.5-fold decrease in pulmonary KC mRNA expression.

\section{Discussion}

It is now generally accepted that human disease can be a multi-stage, multihit process in which various cells within a target organ contribute to disease pathology. In addition to cell-cell interactions within a single organ, recent studies have begun to highlight the importance of more complex interactions, including those between multiple organs. One example of such inter-organ interaction in the setting of alcohol exposure is the critical link between the 
Figure 4.4: Etanercept protects against enhanced cytokine levels in the BALF without concomitant protection against liver injury.

(A) Representative photomicrographs of liver tissue (200x; top) and lung tissue (400x; bottom) after hematoxylin \& eosin staining. (B) Fold change in indices of liver injury (ALT, AST), plasma and pulmonary cytokine levels, and mRNA expression in the liver and lung. Data are shown as $+/-$ SEM ${ }^{a}, p<0.05$ compared to no etanercept. 
A

H\&E
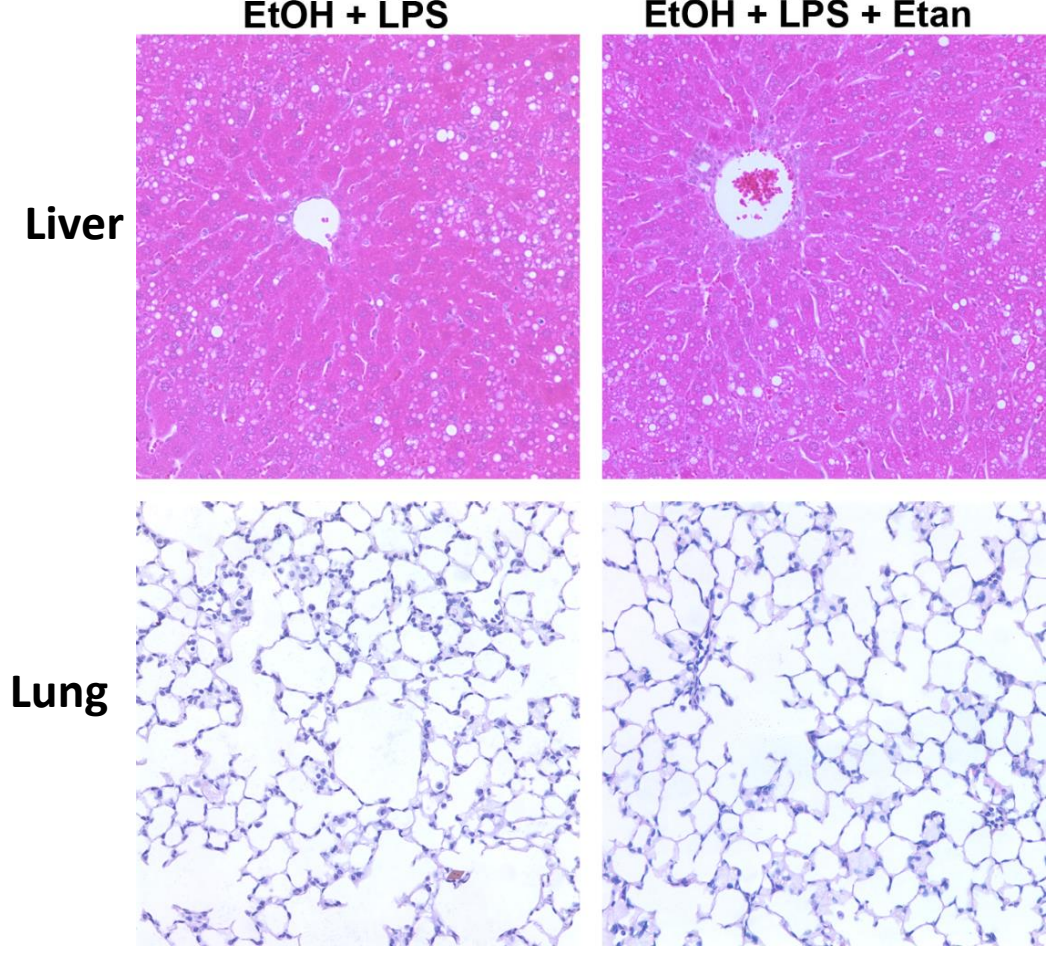

B

EtOH + LPS vs. EtOH + LPS + Etan

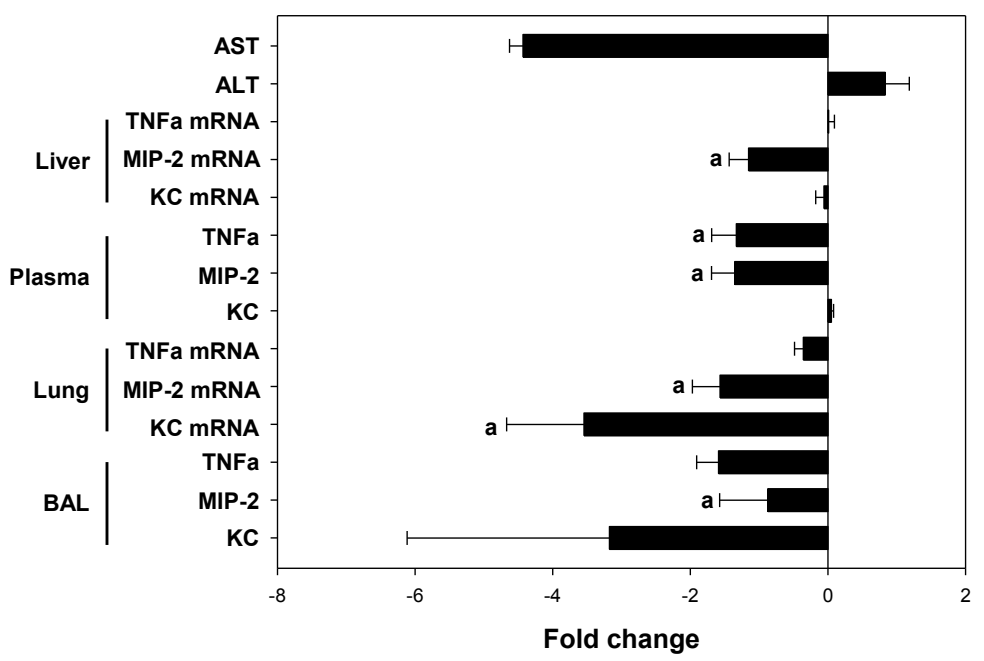


intestine and the liver, or the gut-liver axis (156). Indeed, a myriad of experimental and clinical studies have demonstrated that alcohol-induced gut dysbiosis and subsequent impairment of the gut barrier results in the release of gut-derived toxins (e.g. LPS) and endotoxemia (157-159). Studies using antibiotics (137), germ-free mouse models (160), and probiotics (161) have confirmed that endotoxemia caused by alcohol consumption drives injury in experimental models of ALD. Today, the gut-liver axis and enteric dysbiosis is a widely-accepted component in the development of ALD as well as other liver disease $(156,162)$.

This group is particularly interested in potential interactions between the liver and lung, i.e. the liver-lung axis, in the context of alcohol exposure. As mentioned in the introduction, liver disease is associated with increased incidence of acute lung injury and enhanced mortality. These and other findings have suggested a potential interaction between the liver and lung that may represent an important aspect of the pathophysiology of acute lung injury, particularly in the setting of sepsis. Bringham and collaborators tested the possible interaction between the liver and lung in a study performed in an in situ perfused piglet preparation (152). Their model revealed that hypoxemia and pulmonary edema were only observed when the liver and lung circulations were connected. Their results indicate that while endotoxemia could directly cause pulmonary vasoconstriction and leukocyte sequestration, interaction between the liver and lung was required for severe inflammatory response and oxidative injury 
to the lung. Additional studies have also shown that pulmonary injury induced by systemic endotoxin can be altered by mediators released from the liver (e.g. TNFa) $(84,152)$. Unfortunately, few studies have investigated the potential interactions between the liver and lung in the setting of chronic alcohol exposure. Therefore the goal of this study was to characterize a model of concomitant liver and lung injury caused by LPS after chronic ethanol administration. The LieberDecarli model of chronic alcohol administration was chosen for this study because this model has been used previously to study effects of alcohol exposure on both the liver (163-167) and lung (62, 168, 169), albeit separately. Systemic LPS administration served as an inflammatory stimulus to induce hepatic inflammation and an ARDS-like phenotype in the lung.

The effects of the Lieber-DeCarli rodent model of ethanol exposure on the liver is well-characterized and is predominated by hepatic steatosis (163). Six weeks of exposure to the Lieber-Decarli diet does not cause overt liver injury or hepatic inflammation (163), but sensitizes the liver to other toxic stimuli. In this study, LPS served as an inflammatory insult that induces hepatic inflammation and injury. In this 2-hit model, alcohol pre-exposure enhanced liver injury and inflammation, which corroborates previous findings in this model. Ethanol exposure has been shown to prime immune cells, including Kupffer cells, to release cytotoxic mediators including TNF $\alpha$ after stimulus $(77,170,171)$. In this study enhanced liver injury correlated with enhanced mRNA expression and plasma levels of TNFa in response to LPS, which previous studies have shown to be predominantly derived from Kupffer cells (172). 
Acute lung injury is associated with increased release of pro-inflammatory cytokines (173) in the lung and neutrophil migration into the interstitium (174). In this study, ethanol pre-exposure enhanced alveolar hemorrhage and neutrophil infiltration caused by LPS. Studies have shown that neutrophil migration into the lung is at least partially mediated by the chemokines MIP-2 and KC (175-177). Indeed, both MIP-2 and KC have been linked to acute lung injury caused by systemic LPS $(178,179)$ by mediating neutrophil recruitment via activation of CXCR2 (180). The work presented here shows that LPS-induced MIP-2 and KC expression in the lung was enhanced by ethanol exposure. Furthermore, increases in these chemokines correlated with increased neutrophil recruitment. Interestingly, increased expression of MIP-2 and KC in the lung and BAL have been shown to be downstream of TNFa signaling (88), which was not enhanced by ethanol pre-exposure in the lung in our model. In contrast, hepatic TNFa mRNA expression and systemic levels of TNFa were enhanced by the combination of ethanol + LPS. As mentioned previously, the role of Kupffer cellderived TNFa in liver injury is well recognized (181-183), however hepaticderived TNFa has also been shown to contribute to pulmonary injury. A study by Kono et al demonstrated that depletion of hepatic Kupffer cells protected against pulmonary inflammation and injury and correlated with decreased TNFa expression (183). These findings suggest that systemic inflammation mediated by increased release of TNFa by hepatic macrophages (i.e. Kupffer cells), may be partially responsible for driving pulmonary MIP-2 and KC expression, and subsequent pulmonary injury, as presented here. 
In order to determine the role of systemic/hepatic TNFa in enhanced pulmonary injury, the effect of depleting systemic TNFa with etanercept was determined. Etanercept is a fusion protein of the human p75 TNF receptor and the Fc portion of human IgG1. This TNFa binding antibody has been approved by the FDA for treatment of rheumatoid arthritis and psoriasis. In the work presented here, etanercept administration resulted in a 1.7 and 1.9 fold decrease in systemic and pulmonary TNFa levels, respectively, compared to animals that were exposed to ethanol + LPS in the absence of etanercept. This incomplete blocking of systemic and pulmonary TNFa was likely due to the robust increase in TNF $\alpha$ caused by systemic LPS administration, particularly in the plasma; in fact, LPS administration caused more than a 10 fold increase in systemic TNFa levels compared to their vehicle-treated, ethanol-fed counterparts. Despite the lack of complete TNFa knockdown, etanercept decreased TNFa levels in the plasma by $>100 \mathrm{pg} / \mathrm{mL}$.

Etanercept under these conditions provided partial protection against inflammatory injury in the lung caused by the combination of ethanol and LPS. Etanercept administration blunted enhanced MIP-2 and KC levels in the lung, resulting in a 1.6 and 3.5 fold decrease, respectively. These results support previous findings that TNF $\alpha$ signaling was upstream of enhanced MIP-2 expression in experimental ARDS (184). The authors of that study showed that TNFR $1^{--}$mice were partially protected against increased MIP-2 expression in a model of acute lung injury (184). Whereas the protective effect of TNFa depletion was significant, it was not complete. Indeed, TNFa is likely one of 
several factors that may contribute to the 'alcoholic lung phenotype. For example, PAI-1 has been linked to liver and lung injury $(29,50,185)$ and was differentially affected by ethanol pre-exposure in this model. Additionally, oxidative stress and transitional tissue remodeling are known to play important roles in liver injury and inflammation. Therefore, complete protection against injury in either organ will likely require multiple interventions or an intervention that protects against multiple mechanisms of injury (i.e., Kupffer cell blockade). Nevertheless, these results establish a strong link between systemic TNFa and lung damage in this model of alcohol-enhanced ARDS.

As mentioned above, the priming effect of ethanol on the hepatic inflammatory response is relatively well established. In contrast, the effect of ethanol exposure on lung has predominantly focused on impaired bacterial clearance and subsequent increased susceptibility to pulmonary infections (186188). Indeed, others have shown that ethanol preexposure impairs alveolar macrophage function and inflammatory cytokine release $(168,189,190)$. However, there are two important differences between the current study and those that have reported decreased immune response after alcohol. Specifically, the timing of LPS administration has been shown to play an important role in alcohol-induced liver injury. Previous studies have shown that if LPS is administered during acute alcohol intoxication (or w/in 1 day of a bolus dose of alcohol), the hepatic inflammatory response is blunted. In contrast, if LPS is given distal to alcohol intoxication, the inflammatory response is synergized (170). Indeed, most studies that have documented a decrease in pulmonary 
immune response in the context of alcohol exposure used acute models of intoxication in which LPS was administered within hours of alcohol exposure (186, 191-194). Indeed, others have shown that chronic alcohol exposure can enhance pulmonary inflammation post-intoxication. For example, a recent study by Liu et al showed that chronic ethanol exposure enhances the pulmonary inflammatory response caused by insult (195). Additionally, Boe et al showed that chronic ethanol exposure enhances LPS-induced neutrophil migration, as was observed in the current study (196). Therefore it is likely that the timing of a stimulus - whether during intoxication or distal to intoxication - plays a critical role in pulmonary response after alcohol exposure. This timing effect could create a 'perfect storm' in the lung that clearance of infection is impaired during alcohol intoxication, but the inflammatory response and normal tissue damage caused by that infection is enhanced later. Such a paradigm would explain the fact that ethanol exposure increases both the incidence of lung infections and the likelihood of developing ARDS in response to a lung infection in the human population.

In conclusion, the current study characterizes a model of alcohol-induced liver and lung sensitization which enhances injury caused by systemic LPS. These data suggest that the pulmonary response to ethanol is dependent on systemic release of TNFa, most likely from the liver (Figure 4.5). Additionally, although ethanol pre-exposure enhanced both liver and lung inflammation, both organs experienced a unique pro-inflammatory cytokine profile indicating that similar, yet 


\section{Figure 4.5 Proposed mechanism of interdependent injury.}

This work supports the hypothesis that systemic TNFa plays an important role in enhanced pulmonary injury caused by the combination of chronic ethanol exposure and LPS. The combination of ethanol and LPS caused significant increases in hepatic TNFa. Interestingly, the combination of ethanol and LPS caused a significant increase in MIP-2 mRNA expression and protein levels in the lung tissue and BAL, respectively. Previous studies have shown that MIP-2 expression in the lung is driven, at least in part, by TNFa signaling. The work here demonstrated that depleting systemic TNFa with etanercept partially protects against increased MIP-2 expression the lung. This protection against MIP-2 correlated with protection against pulmonary injury. 


\section{Ethanol}

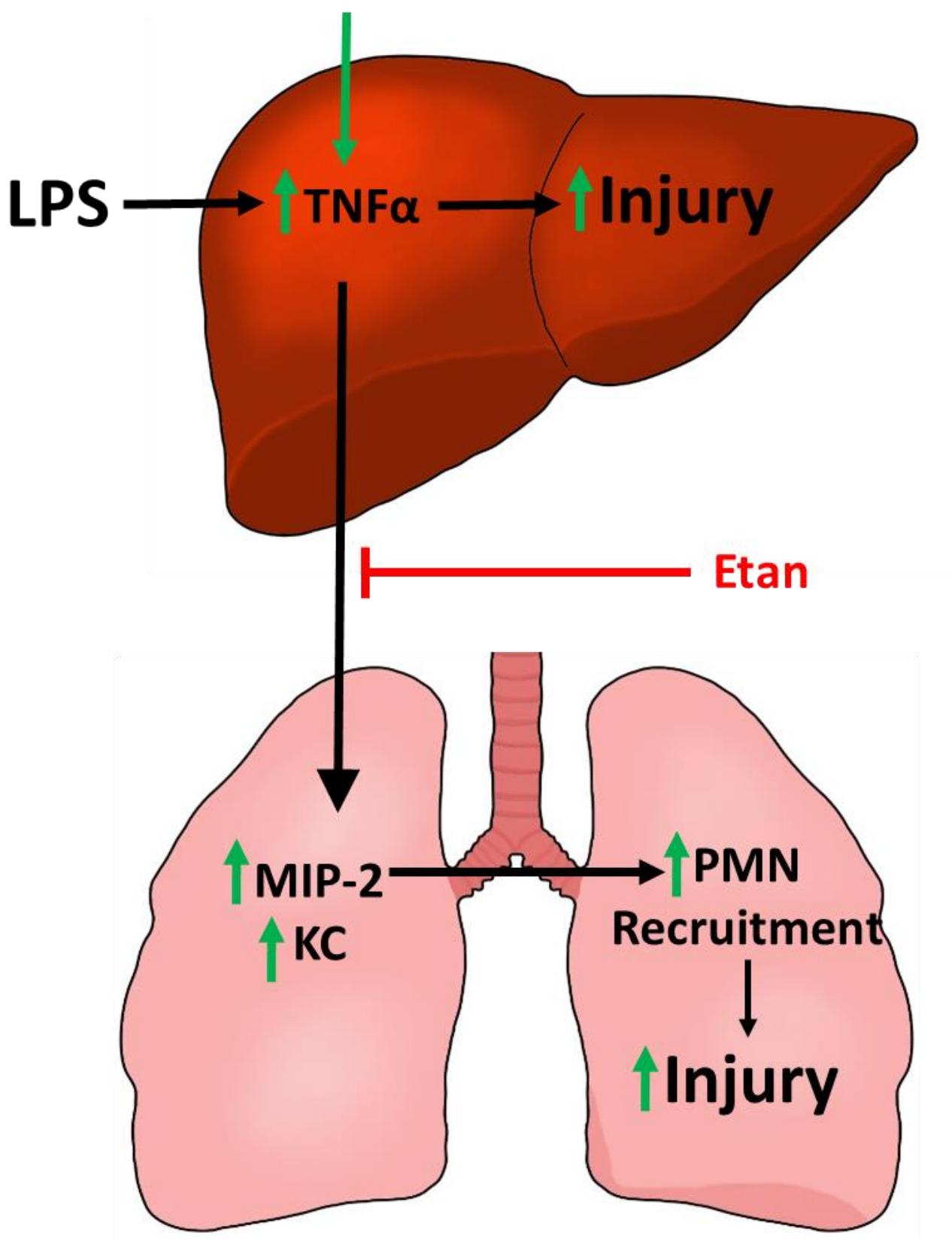


distinct, mechanisms may be contributing to injury in these two organs. Other studies have suggested that injury to the liver and lung may be linked, potentially via systemic mediators such as TNFa. Enhanced MIP-2 and KC expression in the lung, without a concomitant increase in pulmonary TNFa further support a potential role for hepatic-derived TNFa as a mediator of pulmonary injury in this model. Since etanercept is already approved for human use for treatment of rheumatoid arthritis and psoriasis, the translational value of these findings is high. 


\section{CHAPTER V}

\section{ROLE OF THE EXTRACELLULAR MATRIX IN THE LIVER IN A MODEL OF CHRONIC ALCOHOL EXPOSURE}

\section{A. Introduction}

The extracellular matrix (ECM) consists of a diverse group of proteins and sugars including collagens, glycoproteins and proteoglycans. Together, these fibrous macromolecules create a scaffold that provides structure to tissues and a substratum for cells. In addition to their structural role, these ECM components serve as signaling molecules via interactions with cell-surface receptors and impact cell-to-cell communication by binding and retaining soluble mediators including cytokines, chemokines, and growth factors (3). Proteases and protease inhibitors present in the ECM serve as regulators of matrix degradation important for the maintenance of the ECM homeostasis as well as remodeling of the matrix composition in disease (2). Together, the diverse range of the ECM components work bi-directionally with surrounding cells to create a dynamic and responsive microenvironment that regulates cell signaling, recruitment and tissue function.

The role of the ECM is well established in the setting of chronic liver injury, such as alcoholic liver disease (ALD). Indeed, robust changes in the hepatic ECM is the hallmark of later stages of liver disease (fibrosis and cirrhosis) which 
are characterized by dramatic increases in collagen deposition (197). Given the dominance of collagenous changes in the hepatic ECM during fibrosis/cirrhosis, many studies have focused on the mechanisms underlying changes in collagen deposition that lead to hepatic fibrosis. However, the alterations of the hepatic ECM during fibrosis are much more diverse than simply an increase in collagen, and include changes in the deposition and distribution of a myriad of other ECM proteins (e.g., laminin and vitronectin), that may also contribute to fibrogenesis (48).

Hepatic ECM changes are not only more diverse than simply changes to collagen, but also not only relegated solely to fibrogenesis per se. Indeed, it is becoming increasingly understood that the hepatic ECM responds dynamically to stress. For example, changes in the expression of ECM proteins such as fibronectin $(28,90)$, and fibrin $(29,49,50)$ have been observed in models of hepatic inflammation. Importantly, blocking these ECM changes blunts, at least in part, hepatic injury in these models. Therefore, transitional changes to the ECM may contribute to early disease initiation and/or progression long before fibrosis phases of liver disease.

Currently there exists a great need to characterize qualitative and quantitative changes in the ECM protein composition in earlier stages of disease that may be targeted for therapeutic benefit (198). Although previous studies have shown that subtle changes in the ECM may contribute to the development of inflammatory liver injury, the research in this area has thus far been restricted to the hypothesis-driven study of single ECM components. A more 'omic 
approach has been previously hampered by the difficulties associated with the low abundance and insolubility of many ECM proteins. Therefore, a goal of this study was to develop a multi-step liver extraction that would allow for the enrichment and solubilization of ECM proteins. A second goal of this work was to use proteomic analysis to determine global changes in the extracellular matrix in a model of inflammatory liver damage.

\section{B. Experimental procedures}

\section{Animals and treatment}

Animals were exposed to ethanol and/or LPS as described in Chapter II

\section{ECM extraction}

Liver tissue was submitted to a 3-step extraction process that allows sequential isolation of loosely-bound extracellular proteins, cellular proteins, tightly bound extracellular proteins, and highly crosslinked, insoluble proteins to be differentially separated as described in Chapter II.

\section{LC-MS analysis.}

Liver tissue extracts underwent liquid chromatography and mass spec analysis as described in Chapter II

\section{Analysis}

LC-MS data was analyzed as described in Chapter II

\section{Statistical analysis}

Statistical analysis was performed as described in Chapter II. 


\section{Results}

For this study, a previously described 3-step ECM fractionation method was adapted for use with mouse liver tissue (95-97). The ECM isolation method was performed on hepatic tissue of from and a model of ASH which does not cause significant fibrosis. Three sequential extraction steps were used in order to reduce cellular protein contamination and allow for greater resolution of low expressing ECM proteins (Figure 5.1).

The first step of the extraction involved treatment with $0.5 \mathrm{M} \mathrm{NaCl}$. This step removes loosely-bound proteins by displacing polyionic interactions between proteins. The ECM proteins extracted during this step likely include newly formed and/or degraded ECM components and ECM-binding proteins that are loosely associated with the matrix (e.g., proteins that bind to ECM

glycosaminoglycans; (199). Following $\mathrm{NaCl}$ extraction, the remaining liver tissue was decellularized using $1 \%$ SDS. This step solubilizes cytoplasmic and nuclear membranes, allowing for the release of cellular proteins. Decellularization was followed by a denaturing step with $4 \mathrm{M}$ guanidine $\mathrm{HCl}(\mathrm{GnHCl})$ buffer $(200)$. Following the denaturing extraction, an insoluble pellet enriched in highly insoluble, heavily cross-linked matrix proteins (i.e. type I collagens) remains (96). All three extracts as well as the insoluble pellet were subjected to enzymatic deglycosylation with chondroitinase $A B C$, endo-beta-galactosidase, and heparitinase II to remove the side chains chondroitin, keratin, and heparin sulfate of glycoproteins, respectively. This step increases the chance of proper 


\section{Figure 5.1: Scheme of extraction methodology.}

Liver tissue from pair-fed (control) animals and animals exposed to ethanol, LPS, or the combination of ethanol + LPS underwent increasingly vigorous extraction with a $\mathrm{NaCl}$ buffer, SDS buffer, and $\mathrm{GnHCl}$ buffer as described in Materials and Methods. 


\section{Tissue}<smiles>[Te][Te]</smiles>

\section{$\mathrm{NaCl}$ Buffer}

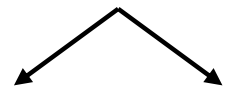

\section{Pellet Supernatant}

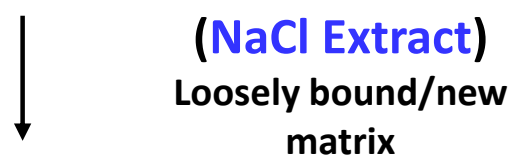

SDS Buffer

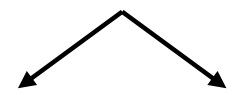

Pellet Supernatant

(SDS Extract)

Cellular components

GnHCl Buffer

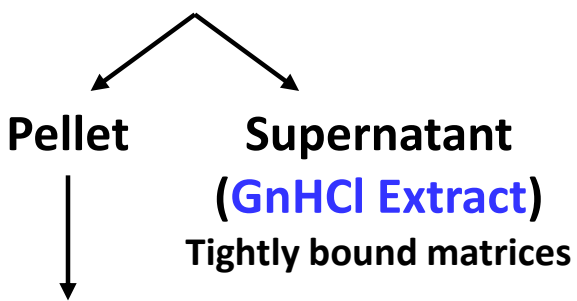

Final pellet

Insoluble fraction 
identification of proteoglycans by mass spectroscopy, which are infamous for being heavily post-translationally modified.

\section{Analysis of LC-MS data}

After LC-MS analysis (see Materials and Methods), Scaffold proteome software was used for visualization of the MS data. Across the four experimental groups, the $\mathrm{NaCl}$ extract contained 588 proteins, the SDS extract contained 511 , the $\mathrm{GnHCl}$ extract contained 585, and the pellet contained 322 proteins. The Scaffold software allows classification of total proteins by gene ontology (GO) annotation. When sorted by $\mathrm{GO}$ annotation, the $\mathrm{NaCl}$ contained 89 extracellular proteins, the SDS extract contained $63, \mathrm{GnHCl}$ contained 84 , and pellet contained 46 extracellular proteins. Additional analysis was only performed on those proteins that were classified as extracellular proteins by GO annotation. The extracellular proteins identified by GO annotation were further categorized into four classes based on their role in the extracellular space as determined by a comprehensive literature search. These four groups include class 1) glycoproteins and proteoglycans, class 2) other ECM-associated proteins class 3) proteases and protease inhibitors and class 4) collagens. If no record could be found supporting the hypothesis that a protein was associated with the ECM, the protein was placed into a fifth group, class 5 ) other proteins. Table 5.1 shows a list of all the proteins from this study that were identified by GO annotation as extracellular, the classification of the extracellular proteins, and references supporting their association with the ECM. 
Table 5.1: Extracellular proteins

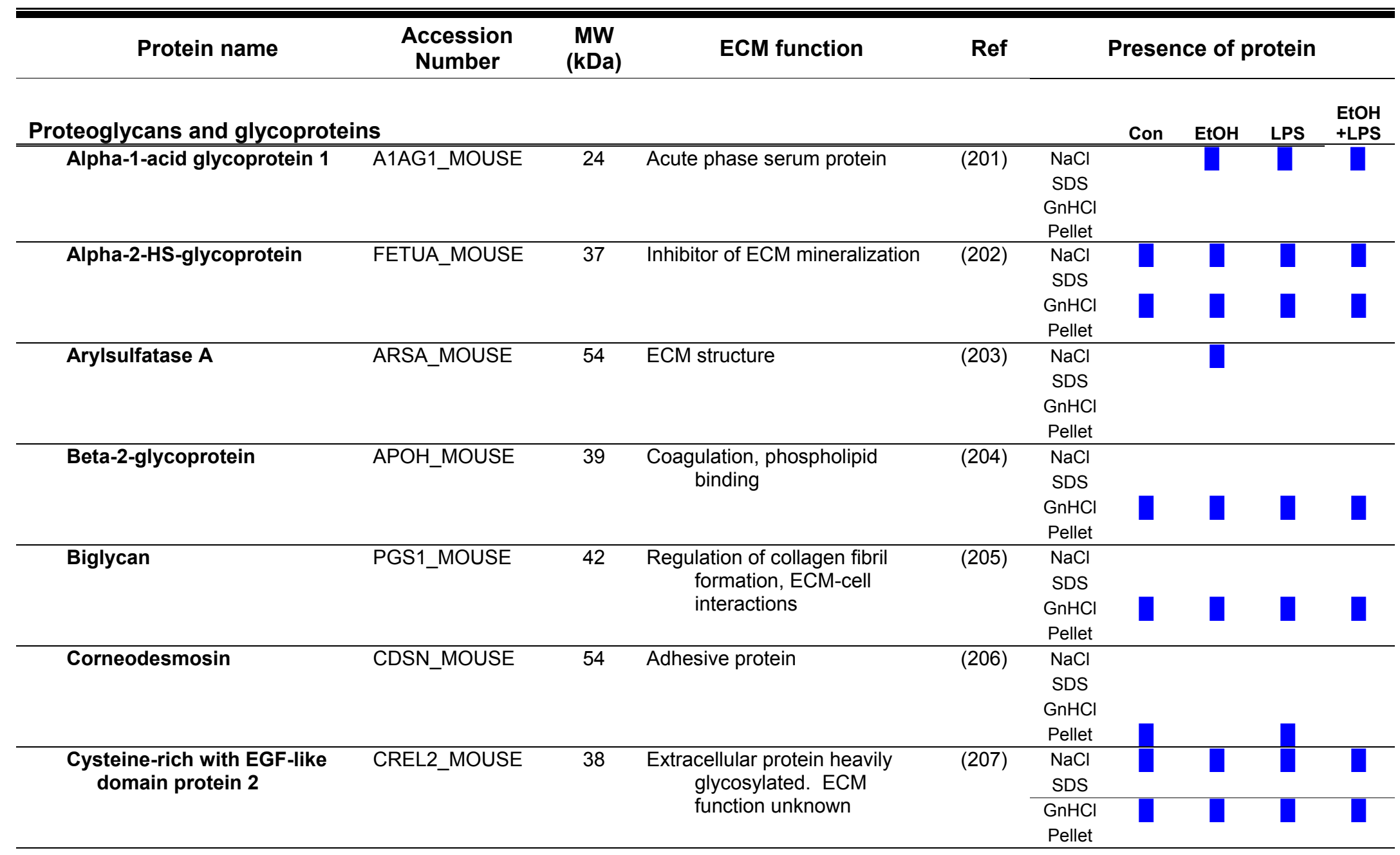




\begin{tabular}{|c|c|c|c|c|c|}
\hline Decorin & PGS2_MOUSE & 40 & $\begin{array}{l}\text { ECM-cell interactions, ECM } \\
\text { assembly }\end{array}$ & $(208)$ & $\begin{array}{c}\mathrm{NaCl} \\
\mathrm{SDS} \\
\mathrm{GnHCl} \\
\text { Pellet }\end{array}$ \\
\hline Dermatopontin & DERM_MOUSE & 24 & $\begin{array}{l}\text { ECM-cell interactions, ECM } \\
\text { assembly }\end{array}$ & $(209)$ & $\begin{array}{c}\mathrm{NaCl} \\
\mathrm{SDS} \\
\mathrm{GnHCl} \\
\text { Pellet }\end{array}$ \\
\hline Dystroglycan & DAG1_MOUSE & 97 & $\begin{array}{c}\text { Matrix organization, basement } \\
\text { membrane assembly }\end{array}$ & $(210)$ & $\begin{array}{c}\mathrm{NaCl} \\
\mathrm{SDS} \\
\mathrm{GnHCl} \\
\text { Pellet }\end{array}$ \\
\hline \multirow[t]{4}{*}{ Fibrinogen alpha chain } & E9PV24_MOUSE & 87 & \multirow{4}{*}{$\begin{array}{l}\text { Hemostasis, cell signaling } \\
\text { (fibrin(ogen) alpha chain) }\end{array}$} & $(211)$ & $\mathrm{NaCl}$ \\
\hline & & & & & SDS \\
\hline & & & & & $\mathrm{GnHCl}$ \\
\hline & & & & & Pellet \\
\hline Fibrinogen beta chain & FIBB_MOUSE & 55 & Hemostasis, cell signaling & $(211)$ & $\begin{array}{l}\mathrm{NaCl} \\
\mathrm{SDS} \\
\mathrm{GnHCl} \\
\text { Pellet }\end{array}$ \\
\hline Fibrinogen gamma chain & FIBG_MOUSE & 49 & $\begin{array}{c}\text { Hemostasis, fibrinogen } \\
\text { polymerization }\end{array}$ & $(212)$ & $\begin{array}{c}\mathrm{NaCl} \\
\mathrm{SDS} \\
\mathrm{GnHCl} \\
\text { Pellet }\end{array}$ \\
\hline Fibronectin & FINC_MOUSE & 273 & $\begin{array}{l}\text { Scaffolding, ECM organization, } \\
\text { regulation of ECM-cell } \\
\text { interactions }\end{array}$ & $(213)$ & $\begin{array}{l}\mathrm{NaCl} \\
\mathrm{SDS} \\
\mathrm{GnHCl} \\
\text { Pellet }\end{array}$ \\
\hline Galectin-1 & LEG1_MOUSE & 15 & $\begin{array}{l}\text { Matrix crosslinking, matrix } \\
\text { organization }\end{array}$ & $(214)$ & $\begin{array}{l}\mathrm{NaCl} \\
\mathrm{SDS} \\
\mathrm{GnHCl} \\
\text { Pellet }\end{array}$ \\
\hline Galectin-3-binding protein & LG3BP_MOUSE & 64 & Cell-matrix adhesion & $(215)$ & $\begin{array}{c}\mathrm{NaCl} \\
\mathrm{SDS} \\
\mathrm{GnHCl} \\
\text { Pellet }\end{array}$ \\
\hline
\end{tabular}




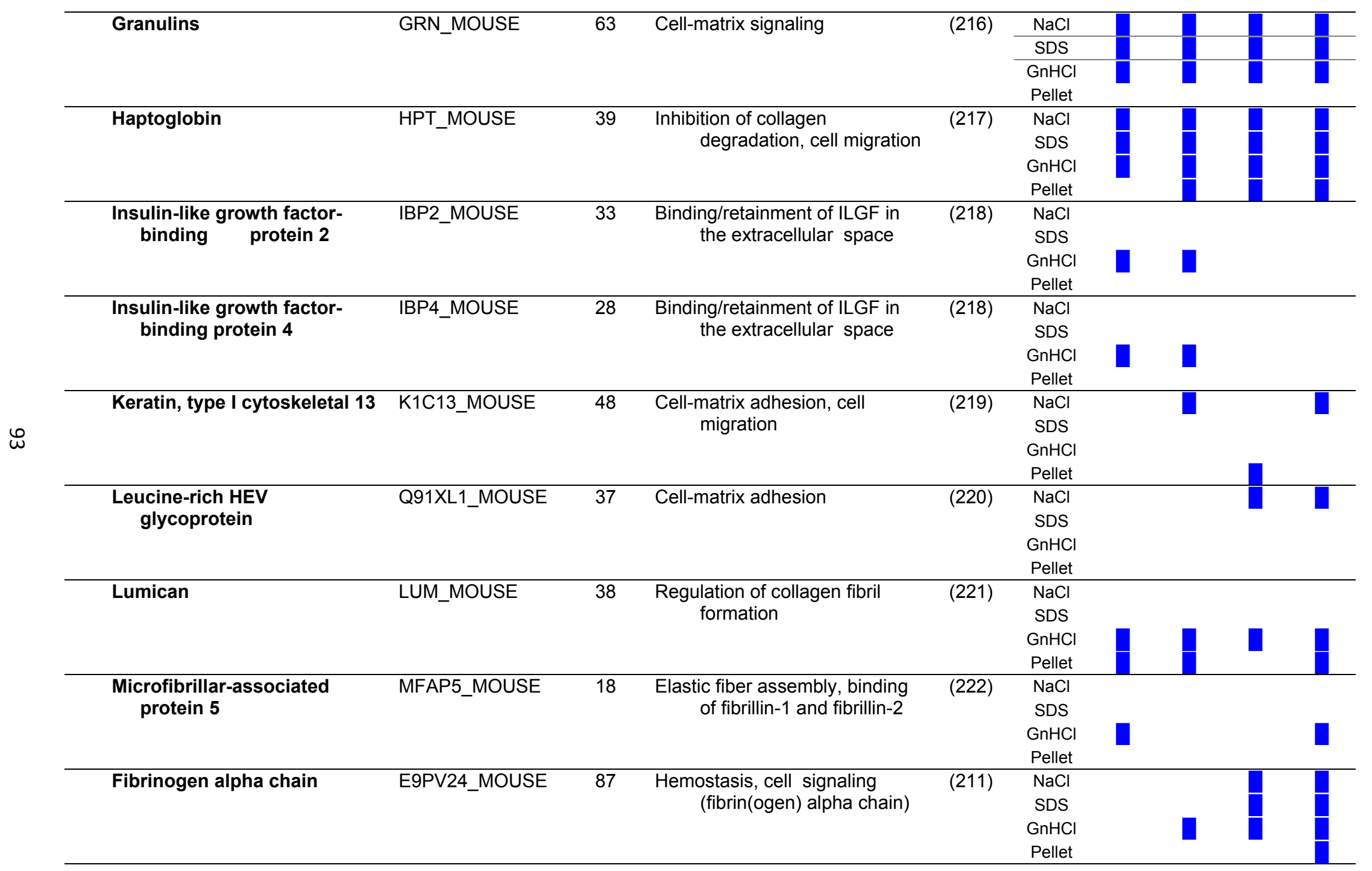




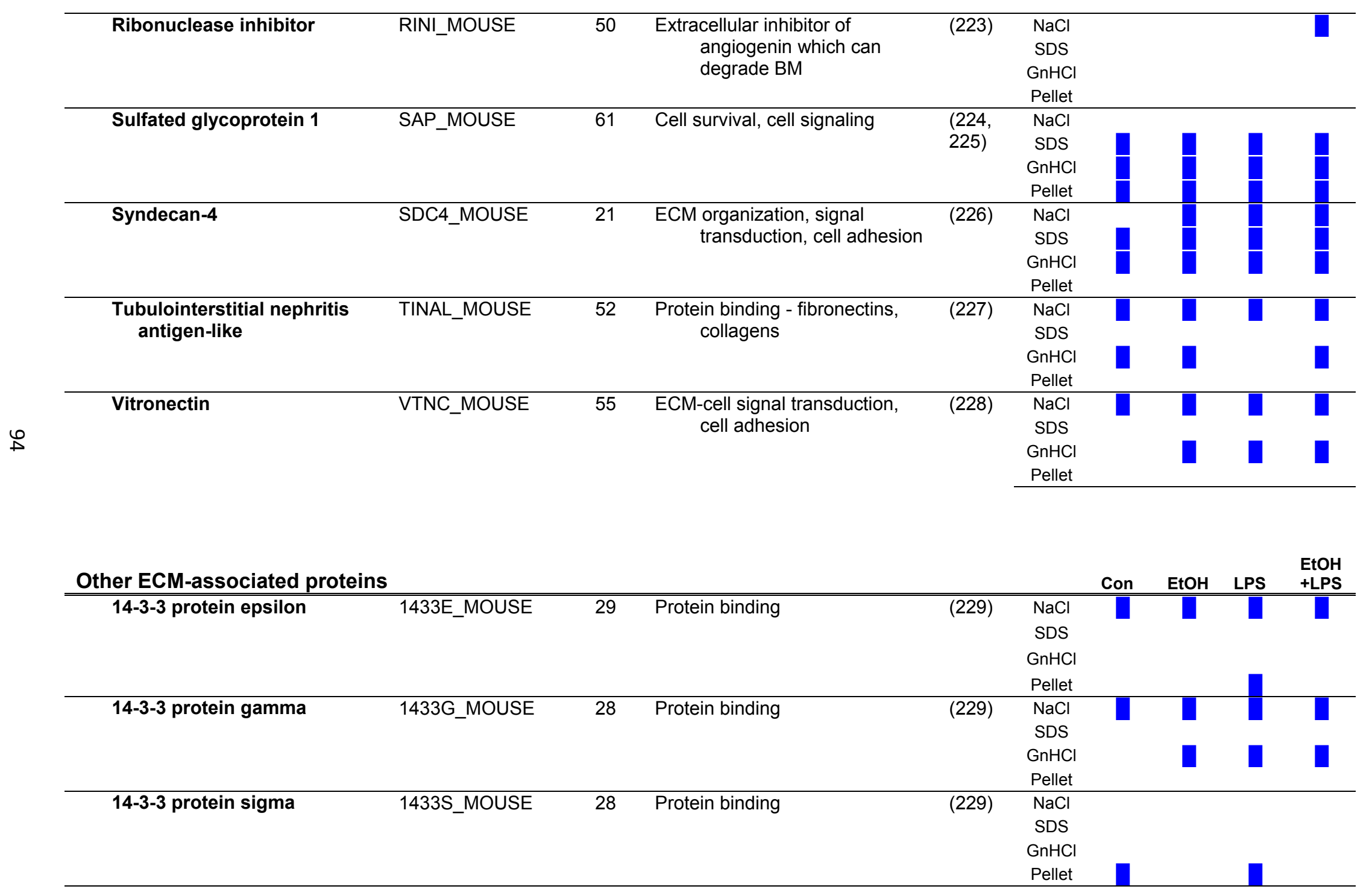




\begin{tabular}{|c|c|c|c|c|c|}
\hline 14-3-3 protein zeta/delta & 1433Z_MOUSE & 28 & Protein binding & $(229)$ & $\begin{array}{c}\mathrm{NaCl} \\
\mathrm{SDS} \\
\mathrm{GnHCl} \\
\text { Pellet }\end{array}$ \\
\hline Actin, cytoplasmic 1 & ACTB_MOUSE & 42 & Cortical cytoskeleton & $\begin{array}{l}(230 \\
231)\end{array}$ & $\begin{array}{c}\mathrm{NaCl} \\
\mathrm{SDS} \\
\mathrm{GnHCl} \\
\text { Pellet }\end{array}$ \\
\hline Actin, cytoplasmic 2 & ACTG_MOUSE & 42 & Cortical cytoskeleton & $\begin{array}{l}(230 \\
231)\end{array}$ & $\begin{array}{c}\mathrm{NaCl} \\
\mathrm{SDS} \\
\mathrm{GnHCl} \\
\text { Pellet }\end{array}$ \\
\hline $\begin{array}{l}\text { Alpha-2-macroglobulin } \\
\text { receptor- } \\
\text { associated protein }\end{array}$ & AMRP_MOUSE & 42 & $\begin{array}{l}\text { Protein binding, soluble } \\
\text { mediator internalization }\end{array}$ & $(232)$ & $\begin{array}{c}\mathrm{NaCl} \\
\mathrm{SDS} \\
\mathrm{GnHCl} \\
\text { Pellet }\end{array}$ \\
\hline Alpha-actinin-4 & ACTN4_MOUSE & 105 & Focal adhesion, cell adhesion & $\begin{array}{l}(233 \\
234)\end{array}$ & $\begin{array}{c}\mathrm{NaCl} \\
\mathrm{SDS} \\
\mathrm{GnHCl} \\
\text { Pellet }\end{array}$ \\
\hline Annexin A1 & ANXA1_MOUSE & 39 & ECM binding, fibrinolysis & $(235)$ & $\begin{array}{c}\mathrm{NaCl} \\
\mathrm{SDS} \\
\mathrm{GnHCl} \\
\text { Pellet }\end{array}$ \\
\hline Annexin A2 & ANXA2_MOUSE & 39 & ECM binding, fibrinolysis & $(236)$ & $\begin{array}{c}\mathrm{NaCl} \\
\mathrm{SDS} \\
\mathrm{GnHCl} \\
\text { Pellet }\end{array}$ \\
\hline Annexin A5 & ANXA5_MOUSE & 36 & ECM binding (collagen) & $(237)$ & $\begin{array}{c}\mathrm{NaCl} \\
\mathrm{SDS} \\
\mathrm{GnHCl} \\
\text { Pellet }\end{array}$ \\
\hline Apolipoprotein A-I & APOA1_MOUSE & 31 & $\begin{array}{l}\text { ECM binding (collagen, } \\
\text { fibronectin) }\end{array}$ & $(238)$ & $\begin{array}{c}\mathrm{NaCl} \\
\mathrm{SDS} \\
\mathrm{GnHCl} \\
\text { Pellet }\end{array}$ \\
\hline
\end{tabular}




\begin{tabular}{|c|c|c|c|c|c|}
\hline Apolipoprotein A-IV & APOA4_MOUSE & 45 & Lipid metabolism & (239) & $\begin{array}{c}\mathrm{NaCl} \\
\mathrm{SDS} \\
\mathrm{GnHCl} \\
\text { Pellet }\end{array}$ \\
\hline Apolipoprotein E & APOE_MOUSE & 36 & Lipid metabolism, cell signaling & $(240)$ & $\begin{array}{c}\mathrm{NaCl} \\
\mathrm{SDS} \\
\mathrm{GnHCl} \\
\text { Pellet }\end{array}$ \\
\hline Apolipoprotein O-like & APOOL_MOUSE & 29 & & & $\begin{array}{c}\mathrm{NaCl} \\
\mathrm{SDS} \\
\mathrm{GnHCl} \\
\text { Pellet }\end{array}$ \\
\hline Beta-2-microglobulin & B2MG_MOUSE & 14 & $\begin{array}{l}\text { Protein binding, cell-ECM } \\
\text { interaction }\end{array}$ & $(241)$ & $\begin{array}{c}\mathrm{NaCl} \\
\mathrm{SDS} \\
\mathrm{GnHCl} \\
\text { Pellet }\end{array}$ \\
\hline Calreticulin & CALR_MOUSE & 48 & $\begin{array}{l}\text { Protein binding (collagens, } \\
\text { laminin) }\end{array}$ & $(242)$ & $\begin{array}{c}\mathrm{NaCl} \\
\mathrm{SDS} \\
\mathrm{GnHCl} \\
\text { Pellet }\end{array}$ \\
\hline Calumenin & CALU_MOUSE & 37 & $\begin{array}{l}\text { Pprotein binding, stabilization of } \\
\text { fibulin-1 }\end{array}$ & $(243)$ & $\begin{array}{c}\mathrm{NaCl} \\
\mathrm{SDS} \\
\mathrm{GnHCl} \\
\text { Pellet }\end{array}$ \\
\hline Carbonic anhydrase 2 & CAH2_MOUSE & 29 & $\begin{array}{l}\mathrm{pH} \text { regulation of ECM (other } \\
\text { isozymes) }\end{array}$ & $(244)$ & $\begin{array}{c}\mathrm{NaCl} \\
\mathrm{SDS} \\
\mathrm{GnHCl} \\
\text { Pellet }\end{array}$ \\
\hline Ceruloplasmin & CERU_MOUSE & 121 & Iron, copper binding & $(245)$ & $\begin{array}{c}\mathrm{NaCl} \\
\mathrm{SDS} \\
\mathrm{GnHCl} \\
\text { Pellet }\end{array}$ \\
\hline Chitinase-like protein 3 & CHIL3_MOUSE & 44 & ECM turnover & $(246)$ & $\begin{array}{c}\mathrm{NaCl} \\
\mathrm{SDS} \\
\mathrm{GnHCl} \\
\text { Pellet }\end{array}$ \\
\hline
\end{tabular}




\begin{tabular}{|c|c|c|c|c|c|}
\hline Clusterin & CLUS_MOUSE & 52 & Extracellular protein chaperone & $(247)$ & $\begin{array}{c}\mathrm{NaCl} \\
\mathrm{SDS} \\
\mathrm{GnHCl} \\
\text { Pellet }\end{array}$ \\
\hline Cofilin-1 & COF1_MOUSE & 19 & $\begin{array}{l}\text { Cortical cytoskeleton, cell } \\
\text { motility }\end{array}$ & $(248)$ & $\begin{array}{c}\mathrm{NaCl} \\
\mathrm{SDS} \\
\mathrm{GnHCl} \\
\text { Pellet }\end{array}$ \\
\hline C-reactive protein & CRP_MOUSE & 25 & Protein binding (fibronectin) & (249) & $\begin{array}{c}\mathrm{NaCl} \\
\mathrm{SDS} \\
\mathrm{GnHCl} \\
\text { Pellet }\end{array}$ \\
\hline C-X-C motif chemokine 9 & CXCL9_MOUSE & 14 & $\begin{array}{l}\text { Soluble mediator (angiostatic } \\
\text { cytokine) }\end{array}$ & $\begin{array}{l}(250, \\
251)\end{array}$ & $\begin{array}{c}\mathrm{NaCl} \\
\mathrm{SDS} \\
\mathrm{GnHCl} \\
\text { Pellet }\end{array}$ \\
\hline Endoplasmin & ENPL_MOUSE & 92 & $\begin{array}{l}\text { Soluble mediator (TLR } \\
\text { signaling) }\end{array}$ & $(252)$ & $\begin{array}{c}\mathrm{NaCl} \\
\mathrm{SDS} \\
\mathrm{GnHCl} \\
\text { Pellet }\end{array}$ \\
\hline $\begin{array}{l}\text { Estradiol 17-beta- } \\
\quad \text { dehydrogenase } 12\end{array}$ & DHB12_MOUSE & 35 & $\begin{array}{l}\text { Protein binding (heparin, } \\
\text { fibronectin, collagen) }\end{array}$ & $(253)$ & $\begin{array}{c}\mathrm{NaCl} \\
\mathrm{SDS} \\
\mathrm{GnHCl} \\
\text { Pellet }\end{array}$ \\
\hline Ferritin heavy chain & FRIH_MOUSE & 21 & Cell migration & (254) & $\begin{array}{c}\mathrm{NaCl} \\
\mathrm{SDS} \\
\mathrm{GnHCl} \\
\text { Pellet }\end{array}$ \\
\hline Ferritin light chain 1 & FRIL1_MOUSE & 21 & Cell migration & $(254)$ & $\begin{array}{c}\mathrm{NaCl} \\
\mathrm{SDS} \\
\mathrm{GnHCl} \\
\text { Pellet }\end{array}$ \\
\hline $\begin{array}{l}\text { Glucose-6-phosphate } \\
\text { isomerase }\end{array}$ & G6PI_MOUSE & 63 & Cell motility & $(255)$ & $\begin{array}{c}\mathrm{NaCl} \\
\mathrm{SDS} \\
\mathrm{GnHCl} \\
\text { Pellet }\end{array}$ \\
\hline
\end{tabular}




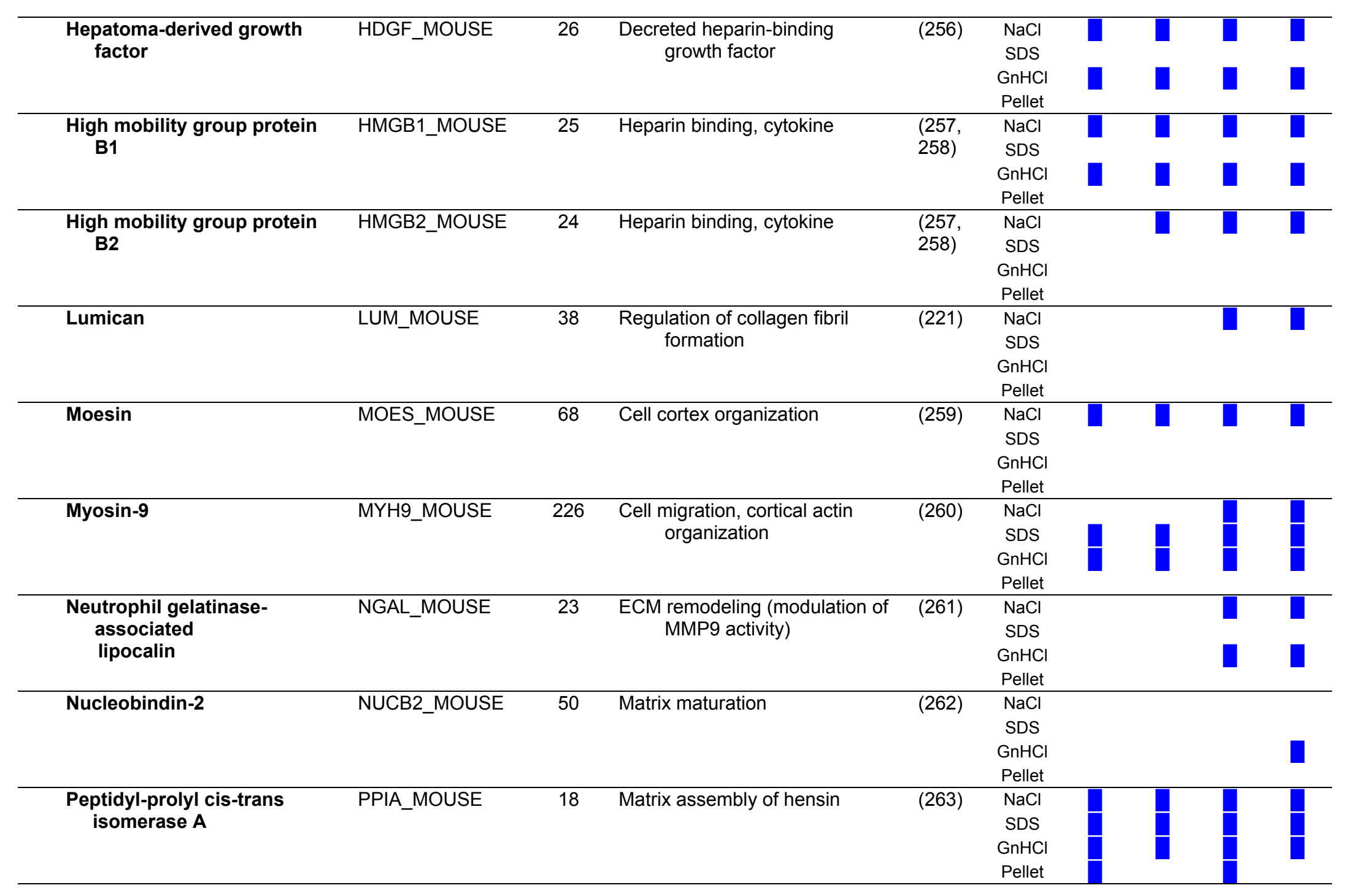




\begin{tabular}{|c|c|c|c|c|c|}
\hline Pro-cathepsin $\mathrm{H}$ & CATH_MOUSE & 37 & Matrix degradation & $(264)$ & $\begin{array}{l}\mathrm{NaCl} \\
\mathrm{SDS} \\
\mathrm{GnHCl} \\
\text { Pellet }\end{array}$ \\
\hline S100-A13 & S10AD_MOUSE & 11 & Calcium binding & $(265)$ & $\begin{array}{c}\mathrm{NaCl} \\
\mathrm{SDS} \\
\mathrm{GnHCl} \\
\text { Pellet }\end{array}$ \\
\hline S100-A9 & & & & & $\begin{array}{c}\mathrm{NaCl} \\
\mathrm{SDS} \\
\mathrm{GnHCl} \\
\text { Pellet }\end{array}$ \\
\hline Serum amyloid A-1 & SAA1_MOUSE & 14 & Cell adhesion & $(266)$ & $\begin{array}{c}\mathrm{NaCl} \\
\mathrm{SDS} \\
\mathrm{GnHCl} \\
\text { Pellet }\end{array}$ \\
\hline Serum amyloid A-2 & SAA2_MOUSE & 14 & Cell adhesion & $(266)$ & $\begin{array}{c}\mathrm{NaCl} \\
\mathrm{SDS} \\
\mathrm{GnHCl} \\
\text { Pellet }\end{array}$ \\
\hline Serum amyloid A-3 & SAA3_MOUSE & 14 & Cell adhesion & $(266)$ & $\begin{array}{c}\mathrm{NaCl} \\
\mathrm{SDS} \\
\mathrm{GnHCl} \\
\text { Pellet }\end{array}$ \\
\hline Serum amyloid P-component & SAMP_MOUSE & 26 & $\begin{array}{r}\text { Protein binding (type IV } \\
\text { collagen, laminin) }\end{array}$ & $\begin{array}{l}(267, \\
268)\end{array}$ & $\begin{array}{c}\mathrm{NaCl} \\
\mathrm{SDS} \\
\mathrm{GnHCl} \\
\text { Pellet }\end{array}$ \\
\hline $\begin{array}{l}\text { Superoxide dismutase [Cu- } \\
\quad \mathrm{Zn}]\end{array}$ & SODC_MOUSE & 16 & $\begin{array}{l}\text { Protein binding } \\
\text { (heparin/heparan sulfate, } \\
\text { type } 1 \text { collage) }\end{array}$ & $(269)$ & $\begin{array}{l}\mathrm{NaCl} \\
\mathrm{SDS} \\
\mathrm{GnHCl} \\
\text { Pellet }\end{array}$ \\
\hline Thioredoxin & THIO_MOUSE & 12 & $\begin{array}{l}\text { Soluble mediator } \\
\text { (immunomodulatory } \\
\text { cytokine) }\end{array}$ & $(258)$ & $\begin{array}{c}\mathrm{NaCl} \\
\mathrm{SDS} \\
\mathrm{GnHCl} \\
\text { Pellet }\end{array}$ \\
\hline
\end{tabular}




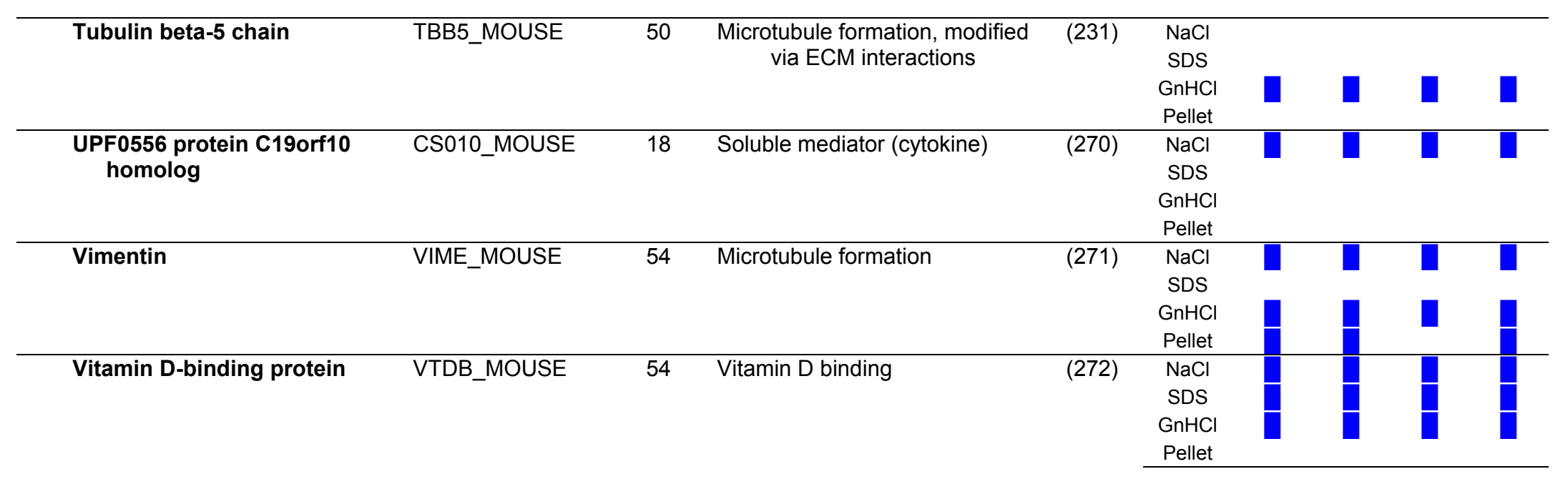

เे

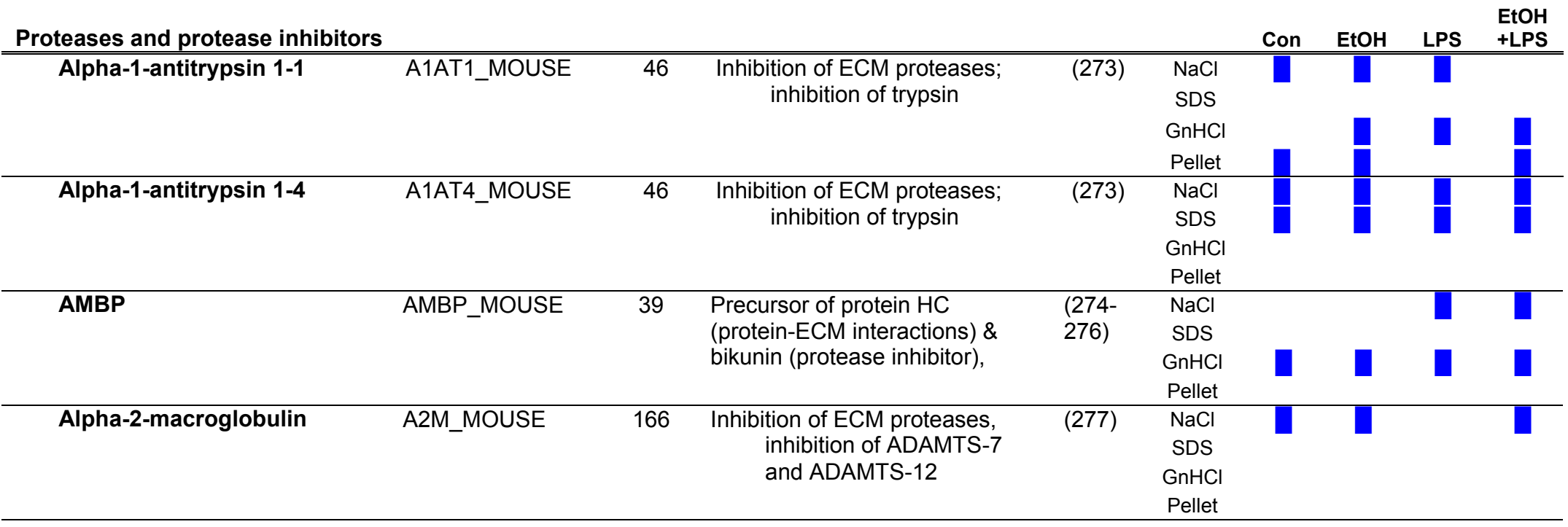




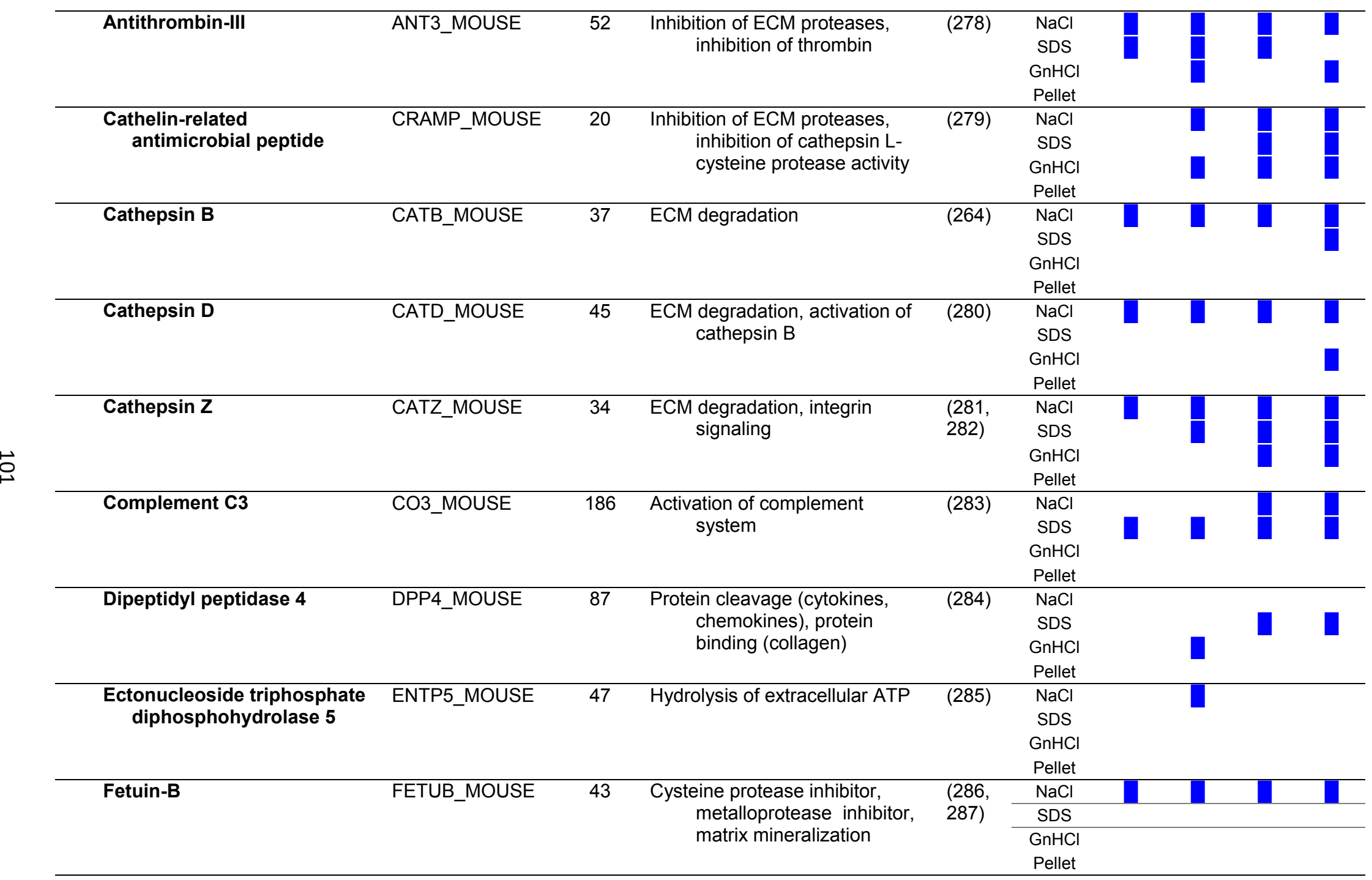




\begin{tabular}{|c|c|c|c|c|c|}
\hline Gelsolin & GELS_MOUSE & 86 & Actin filament capping & $(288)$ & $\begin{array}{c}\mathrm{NaCl} \\
\mathrm{SDS} \\
\mathrm{GnHCl} \\
\text { Pellet }\end{array}$ \\
\hline $\begin{array}{l}\text { Ectonucleoside triphosphate } \\
\text { diphosphohydrolase } 5\end{array}$ & ENTP5_MOUSE & 47 & $\begin{array}{r}\text { Hydrolysis of extracellular } \\
\text { ATPIADP (signaling) }\end{array}$ & $(286)$ & $\begin{array}{c}\mathrm{NaCl} \\
\mathrm{SDS} \\
\mathrm{GnHCl} \\
\text { Pellet }\end{array}$ \\
\hline Hemopexin & HEMO_MOUSE & 51 & Heme binding & $(289)$ & $\begin{array}{c}\mathrm{NaCl} \\
\mathrm{SDS} \\
\mathrm{GnHCl} \\
\text { Pellet }\end{array}$ \\
\hline Insulin-degrading enzyme & IDE_MOUSE & 118 & Metalloendopeptidase & $(290)$ & $\begin{array}{c}\mathrm{NaCl} \\
\mathrm{SDS} \\
\mathrm{GnHCl} \\
\text { Pellet }\end{array}$ \\
\hline $\begin{array}{l}\text { Inter alpha-trypsin inhibitor, } \\
\text { heavy chain } 4\end{array}$ & ITIH4_MOUSE & 100 & Chain of ITI (protease inhibitor) & $(291)$ & $\begin{array}{c}\mathrm{NaCl} \\
\mathrm{SDS} \\
\mathrm{GnHCl} \\
\text { Pellet }\end{array}$ \\
\hline Lysozyme C-1 & LYZ1_MOUSE & 17 & $\begin{array}{c}\text { Antibacterial, hydrolysis of } \beta-1 \text {, } \\
\text { 4-glycosidic linkages }\end{array}$ & $(292)$ & $\begin{array}{c}\mathrm{NaCl} \\
\mathrm{SDS} \\
\mathrm{GnHCl} \\
\text { Pellet }\end{array}$ \\
\hline Lysozyme C-2 & LYZ2_MOUSE & 17 & $\begin{array}{l}\text { Antibacterial, hydrolysis of } \beta-1 \text {, } \\
\text { 4-glycosidic linkages }\end{array}$ & $(292)$ & $\begin{array}{c}\mathrm{NaCl} \\
\mathrm{SDS} \\
\mathrm{GnHCl} \\
\text { Pellet }\end{array}$ \\
\hline Murinoglobulin-1 & MUG1_MOUSE & 165 & Protease inhibitor & $\begin{array}{l}(293, \\
294)\end{array}$ & $\begin{array}{c}\mathrm{NaCl} \\
\mathrm{SDS} \\
\mathrm{GnHCl} \\
\text { Pellet }\end{array}$ \\
\hline Myeloid bactenecin (F1) & O08692_MOUSE & 19 & Cysteine protease inhibitor & $(295)$ & $\begin{array}{c}\mathrm{NaCl} \\
\mathrm{SDS} \\
\mathrm{GnHCl} \\
\text { Pellet }\end{array}$ \\
\hline
\end{tabular}




\begin{tabular}{|c|c|c|c|c|c|}
\hline $\begin{array}{l}\text { Phosphatidylethanolamine- } \\
\text { binding protein } 1\end{array}$ & PEBP1_MOUSE & 21 & Serine protease inhibitor & $(296)$ & $\begin{array}{c}\mathrm{NaCl} \\
\mathrm{SDS} \\
\mathrm{GnHCl} \\
\text { Pellet }\end{array}$ \\
\hline Plasminogen & PLMN_MOUSE & 91 & $\begin{array}{l}\text { Precursor of plasmin (serine } \\
\text { protease) }\end{array}$ & $(297)$ & $\begin{array}{c}\mathrm{NaCl} \\
\mathrm{SDS} \\
\mathrm{GnHCl} \\
\text { Pellet }\end{array}$ \\
\hline $\begin{array}{l}\text { Probable carboxypeptidase } \\
\text { PM20D1 }\end{array}$ & P20D1_MOUSE & 56 & & & $\begin{array}{c}\mathrm{NaCl} \\
\mathrm{SDS} \\
\mathrm{GnHCl} \\
\text { Pellet }\end{array}$ \\
\hline Serpinb9 & O08797_MOUSE & 42 & $\begin{array}{r}\text { Serine protease inhibitor, } \\
\text { granzyme inhibitor) }\end{array}$ & $(298)$ & $\begin{array}{c}\mathrm{NaCl} \\
\mathrm{SDS} \\
\mathrm{GnHCl} \\
\text { Pellet }\end{array}$ \\
\hline $\begin{array}{l}\text { Serine protease inhibitor } \\
\text { A3K }\end{array}$ & SPA3K_MOUSE & 47 & $\begin{array}{l}\text { Serine protease inhibitor, } \\
\text { chymotrypsin inhibitor }\end{array}$ & $(299)$ & $\begin{array}{c}\mathrm{NaCl} \\
\mathrm{SDS} \\
\mathrm{GnHCl} \\
\text { Pellet }\end{array}$ \\
\hline $\begin{array}{l}\text { Serine protease inhibitor } \\
\text { A3N }\end{array}$ & SPA3N_MOUSE & 47 & $\begin{array}{l}\text { Serine protease inhibitor, } \\
\text { chymotrypsin inhibitor }\end{array}$ & $(299)$ & $\begin{array}{c}\mathrm{NaCl} \\
\mathrm{SDS} \\
\mathrm{GnHCl} \\
\text { Pellet }\end{array}$ \\
\hline Serpin B5 & SPB5_MOUSE & 42 & Serine protease inhibitor, & $(299)$ & $\begin{array}{c}\mathrm{NaCl} \\
\mathrm{SDS} \\
\mathrm{GnHCl} \\
\text { Pellet }\end{array}$ \\
\hline Thioredoxin & THIO_MOUSE & 12 & Activation of transglutaminase & $(300)$ & $\begin{array}{c}\mathrm{NaCl} \\
\mathrm{SDS} \\
\mathrm{GnHCl} \\
\text { Pellet }\end{array}$ \\
\hline Transthyretin & TTHY_MOUSE & 16 & $\begin{array}{l}\text { Hormone binding protein, } \\
\text { cryptic protease, } \\
\text { peptidase }\end{array}$ & $\begin{array}{l}(301) \\
(302)\end{array}$ & $\begin{array}{c}\mathrm{NaCl} \\
\mathrm{SDS} \\
\mathrm{GnHCl} \\
\text { Pellet }\end{array}$ \\
\hline
\end{tabular}




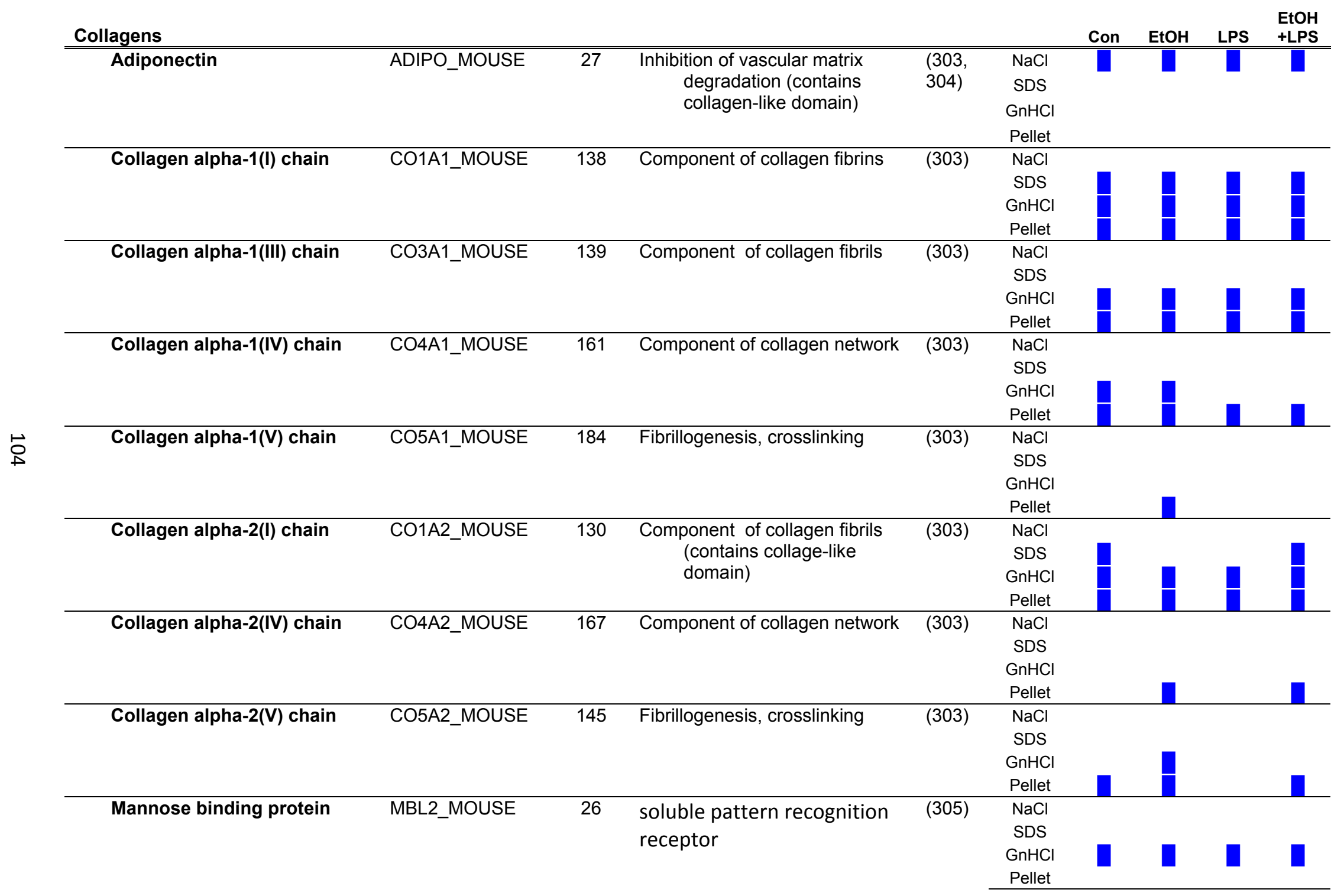


Extracellular - ECM interactions unknown

60 heat shock protein, $\quad$ CH60_MOUSE $\quad 61$

mitochondrial

\begin{tabular}{|c|c|c|}
\hline $\begin{array}{l}78 \text { glucose-regulated } \\
\text { protein }\end{array}$ & GRP78_MOUSE & 72 \\
\hline $\begin{array}{l}\text { Aminoacyl tRNA synthase } \\
\text { complex-interacting } \\
\text { multifunctional } \\
\text { protein } 1\end{array}$ & AIMP1_MOUSE & 34 \\
\hline Arginase-1 & ARGI1_MOUSE & 35 \\
\hline BolA-like protein 1 & BOLA1_MOUSE & 14 \\
\hline BolA-like protein 3 & BOLA3_MOUSE & 12 \\
\hline Carboxylesterase 1C & EST1C_MOUSE & 61 \\
\hline Clathrin heavy chain 1 & CLH1_MOUSE & 192 \\
\hline $\begin{array}{l}\text { Epididymal secretory protein } \\
\text { E1 }\end{array}$ & NPC2_MOUSE & 16 \\
\hline $\begin{array}{l}\text { Group XIIB secretory } \\
\text { phospholipase A2-like } \\
\text { protein }\end{array}$ & Q8VC81_MOUSE & 22 \\
\hline $\begin{array}{l}\text { Heat shock cognate } 71 \\
\text { protein }\end{array}$ & HSP7C_MOUSE & 71 \\
\hline Hepcidin & HEPC_MOUSE & 9 \\
\hline $\begin{array}{l}\text { Hypoxia up-regulated protein } \\
1\end{array}$ & HYOU1_MOUSE & 111 \\
\hline
\end{tabular}




\begin{tabular}{|c|c|c|}
\hline $\begin{array}{l}\text { Interferon-inducible GTPase } \\
\quad 1\end{array}$ & IIGP1_MOUSE & 48 \\
\hline Lactotransferrin & TRFL_MOUSE & 78 \\
\hline $\begin{array}{l}\text { Macrophage migration } \\
\text { inhibitory factor }\end{array}$ & MIF_MOUSE & 13 \\
\hline Major urinary protein 12 & A2CEK7_MOUSE & 21 \\
\hline Major urinary protein 17 & MUP17_MOUSE & 21 \\
\hline Major urinary protein 20 & MUP20_MOUSE & 21 \\
\hline Major urinary protein 3 & MUP3_MOUSE & 21 \\
\hline Major vault protein & MVP_MOUSE & 96 \\
\hline $\begin{array}{l}\text { Monocyte differentiation } \\
\text { antigen CD14 }\end{array}$ & CD14_MOUSE & 39 \\
\hline $\begin{array}{l}\text { Multiple coagulation factor } \\
\text { deficiency protein } 2 \\
\text { homolog }\end{array}$ & MCFD2_MOUSE & 16 \\
\hline Myeloperoxidase & PERM_MOUSE & 81 \\
\hline $\begin{array}{l}\text { Nuclease-sensitive element- } \\
\text { binding protein } 1\end{array}$ & YBOX1_MOUSE & 36 \\
\hline $\begin{array}{l}\text { Polymeric immunoglobulin } \\
\text { receptor }\end{array}$ & PIGR_MOUSE & 85 \\
\hline
\end{tabular}




\begin{tabular}{|c|c|c|}
\hline Protein CREG1 & CREG1_MOUSE & 24 \\
\hline Protein Gm20425 & E9Q035_MOUSE & 108 \\
\hline Pyruvate kinase PKM & KPYM_MOUSE & 58 \\
\hline $\begin{array}{l}\text { RNA binding motif protein, } \\
\text { X-linked-like-1 }\end{array}$ & RMXL1_MOUSE & 42 \\
\hline Serotransferrin & TRFE_MOUSE & 77 \\
\hline Serum albumin & ALBU_MOUSE & 69 \\
\hline $\begin{array}{l}\text { Serum } \\
\text { paraoxonase/arylesterase } \\
1\end{array}$ & PON1_MOUSE & 40 \\
\hline $\begin{array}{l}\text { Translationally-controlled } \\
\text { tumor protein }\end{array}$ & TCTP_MOUSE & 19 \\
\hline $\begin{array}{l}\text { UPF0369 protein C6orf57 } \\
\text { homolog }\end{array}$ & CF057_MOUSE & 12 \\
\hline $\begin{array}{l}\text { Xanthine } \\
\text { dehydrogenase/oxidase }\end{array}$ & XDH_MOUSE & 147 \\
\hline
\end{tabular}




\section{3-step serial extraction creates 4 extracts with distinct protein}

profiles

For initial analysis, the ECM proteins present in each of the four extracts from control animals was compared (Figure 5.2, top) without regard to protein classification. Each extract had a unique extracellular protein profile consisting of not only ECM proteins that were shared among all four extracts (7), but also proteins unique to particular extracts. Indeed, there were $16,3,15$, and 7 proteins unique to the $\mathrm{NaCl}, \mathrm{SDS}, \mathrm{GnHCl}$, and pellet fractions, respectively in livers from control animals. These results indicate that the extraction approach was effective at separating the proteins.

When ECM proteins were compared between treatment groups, interesting patterns of protein presence in the extracts were revealed (Figure 5.3, lower). For example, some proteins (e.g., plasminogen), if present, located consistently to the same extraction fraction across the treatment groups. The effect of treatments on other proteins was more complex than the simple presence and absence. For example, whereas annexin A1 was found in the insoluble pellet in samples from control animals, it was absent in the liver from animals exposed to ethanol or LPS alone; however, the combination of ethanol and LPS caused this protein to accumulate in the $\mathrm{NaCl}$ fraction. Likewise, although fibrin(ogen) gamma chain peptides were found in all treatment groups, the location of these signals was unique to each treatment condition. These 
Figure 5.2: Liver extracts have unique protein profiles based on fraction type and experimental group.

The number of proteins unique to or shared by all four extractions of pair-fed animals is shown (A). Bubble graphs are used to show presence of plasminogen, annexin A1 and fibrin(ogen) gamma chain across the four extracts ( $\mathrm{x}$-axis) and the four experimental groups (y-axis). 

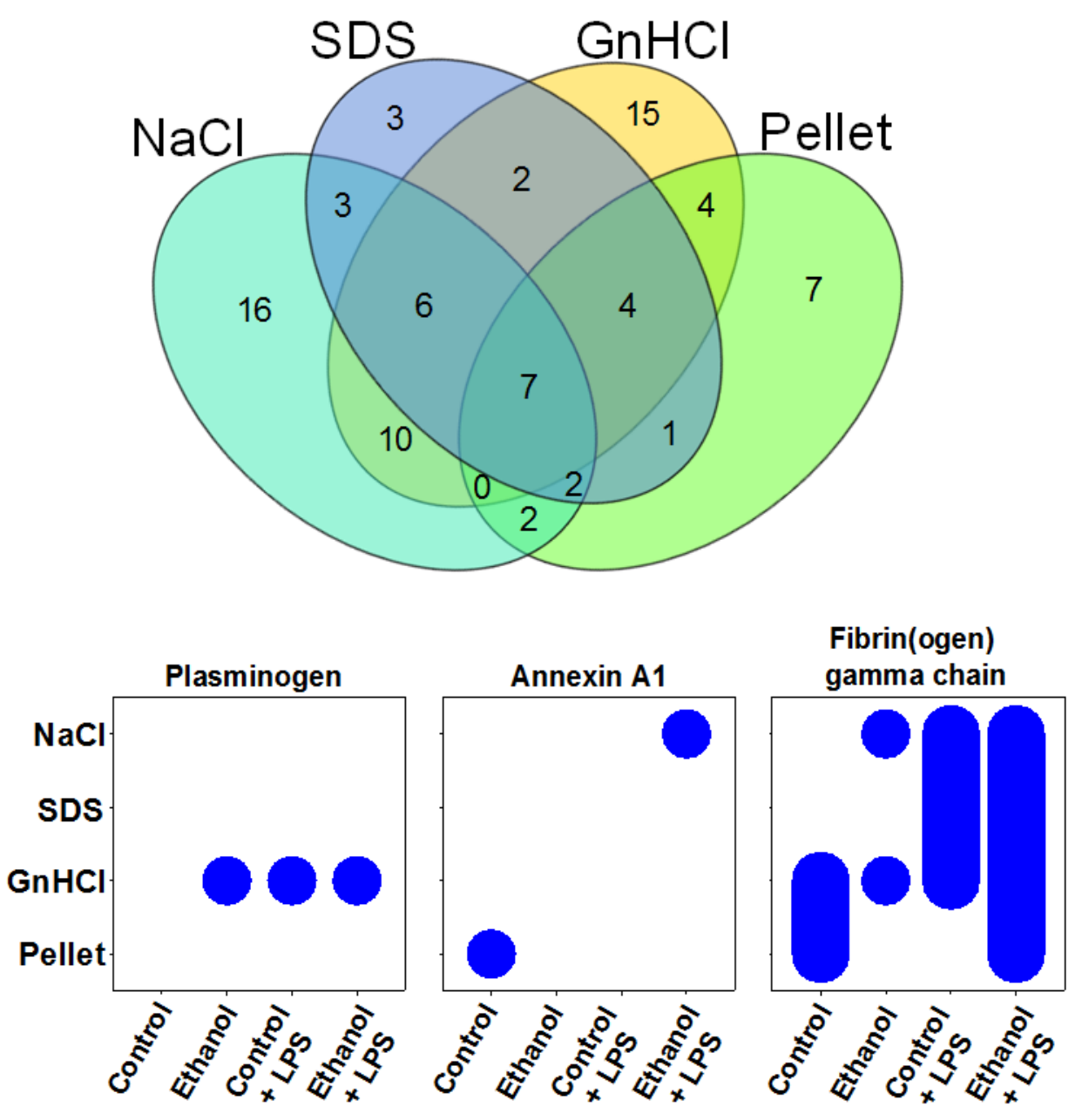
changes likely represent differences in the synthesis, degradation and/or maturity of the ECM proteins.

\section{Qualitative changes to the ECM proteome in response to stress}

The highest abundance of ECM was found in the $\mathrm{GnHCl}$ fraction of the control liver, which was closely followed by the $\mathrm{NaCl}$ fraction. Of the proteins in the $\mathrm{NaCl}$ and $\mathrm{SDS}$ extracts the majority were ECM-associated proteins (class 2;

Figure 5.3); this effect was expected as these are generally loosely associated with the extracellular matrix and are easily solubilized. Additionally, the low abundance of collagens (class 3 ) in the $\mathrm{NaCl}$ and SDS fractions was not surprising as collagens are often tightly cross-linked and require denaturing conditions for solubilization. As expected, the denaturing conditions created by $\mathrm{GnHCl}$ more than doubled the number of proteoglycans and glycoproteins in the $\mathrm{GnHCl}$ extract compared to the $\mathrm{NaCl}$ and SDS fractions. The number of collagens in the $\mathrm{GnHCl}$ extract were also dramatically increased compared to the $\mathrm{NaCl}$ and SDS fractions. The pellet fraction contained the fewest proteins of all four extracts; this fraction has the greatest number of collagens (8) which are known to be highly cross-linked to the point of insolubility (306). These results indicate that the 3 step extraction process was successful in separating and enriching the ECM proteins.

Exposure to ethanol and LPS, alone or combined, did not change the general pattern of proteins found in the various extracts (Figure 5.3), but both tended to increase the number of ECM proteins. For example, ethanol exposure 
Figure 5.3: Ethanol and LPS cause dynamic changes in the matrisome. Each row represents a separate extract and each column represents a separate experimental group comparison. In the left column, the number of proteins unique to the control (blue) or ethanol (yellow) groups and the number of proteins shared between the two experiment groups (green) in toto (Venn diagram) or separated by protein class (stacked bar graph) are shown. Similarly, in the right column, Venn diagrams show the total number of proteins unique to control (blue) or LPS (yellow) group and shared between these two groups (green) and stacked bar graphs show changes in protein number by protein class. The size of the Venn diagrams are proportional to the number of proteins represented. 


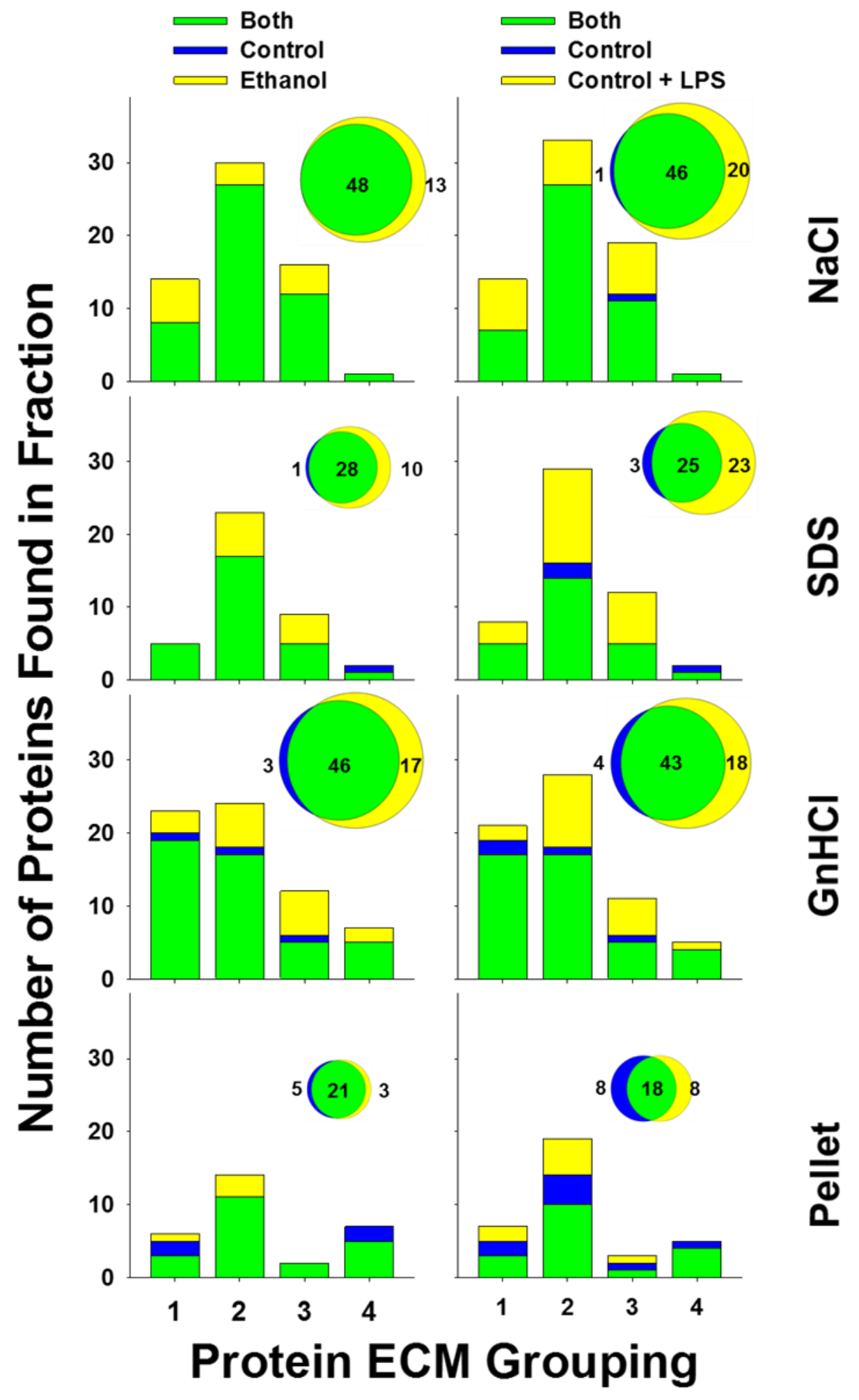


caused a net increase in the number of ECM proteins by $\sim 22 \%$. These changes were spread across the first 3 protein classes [(1) proteoglycans and glycoproteins, (2) ECM associated proteins, and (3) protease and protease inhibitors] with the $\mathrm{NaCl}, \mathrm{SDS}$ and $\mathrm{GnHCl}$ extracts all increasing to a similar extent. The least responsive protein class were the collagens (class 4), which did not show a net increase with ethanol or LPS exposure. Likewise, the pellet fraction responded the least dynamically to ethanol or LPS exposure, and actually showed a net loss in total proteins. Figure 5.4 (left panels), shows the distribution of the proteins in the various extracts between all 4 treatment groups. In addition to proteins that changed in their extraction pattern in response to ethanol (e.g., see Figure 5.2, lower panel), there were 22 proteins whose presence was unique to ethanol exposure compared to control; these include fibrin(ogen) $\alpha$ and $\beta$ chains, cytokeratin 13, vitronectin, plasminogen, high mobility group protein B2, and collagens IVa2 and Va2. Likewise, LPS exposure caused the appearance of several proteins that were unique compared to control. Eighteen of these proteins were shared with ethanol exposure (e.g., fibrin(ogen) $\alpha$ and $\beta$ chains, cytokeratin 13 , vitronectin, plasminogen, high mobility group protein $\mathrm{B} 2$, and collagen IV 2 2), but also 14 that were unique to LPS compared to ethanol, including serpin B5 (maspin), serine protease inhibitor A3N (Serpin A3N), and CXC motif chemokine 9 (CXCL9).

Previous work has shown that ethanol pre-exposure sensitizes the liver to inflammatory injury caused by a second insult (i.e. LPS) $(170,307)$. Furthermore, previous studies have suggested that changes in the ECM 
composition can contribute to the sensitizing effect of ethanol pre-exposure (29, $50,308)$. In this study, the combination of chronic ethanol exposure and a second hit of LPS caused unique changes in the ECM protein profile of the liver. Indeed, the combination of EtOH + LPS resulted in the appearance of 4 unique proteins that were not present in livers from animals exposed to either ethanol or LPS alone, including serum amyloid P and serpine B9.

\section{Quantitative changes to the extracellular proteome caused by stress.}

The composition of extracellular environment is tightly regulated and drastic changes, such as the presence or absence of ECM proteins, can produce functional changes in the surrounding tissue. Therefore, it was not surprising that the presence of the majority (73 proteins) of the extracellular matrix proteins was not altered by any exposure regardless of fraction type. However, several proteins that did not change qualitatively changed in abundance. In order to understand better the more subtle changes on the expression of extracellular proteins in the four fractions, quantitative changes in the expression that were not changed qualitative was determined (Figure 5.4, right panels).

Several proteins change quantitatively in response to ethanol and/or LPS exposure relative to control animals in the livers. Notable ( $>4$-fold increase) changes caused by LPS included ferritin heavy chain (15-fold increase) in the $\mathrm{NaCl}$ fraction, haptoglobin and myosin-9 (11- and 9-fold, respectively) in the SDS fraction, and fibrinogen $\gamma$ chain and haptoglobin (14- and 7-fold, respectively) in

the $\mathrm{GnHCl}$ fraction. Notable changes caused by ethanol include myosin-9 (6- 
Figure 5.4: Liver extracts show quantitative changes in the matrisome. Venn diagrams (left column) show all proteins within an extract and indicate the number that are shared between or that are unique to the four experimental groups. Bubble plots (right column) show quantitative changes in protein expression of proteins that were shared by all four experimental groups. The bubble plots show fold change in protein expression caused by LPS (y-axis), ethanol (x-axis), and the combination of ethanol + LPS (bubble size). Each bubble represents a single extracellular protein; bubble color indicates the protein's class. 

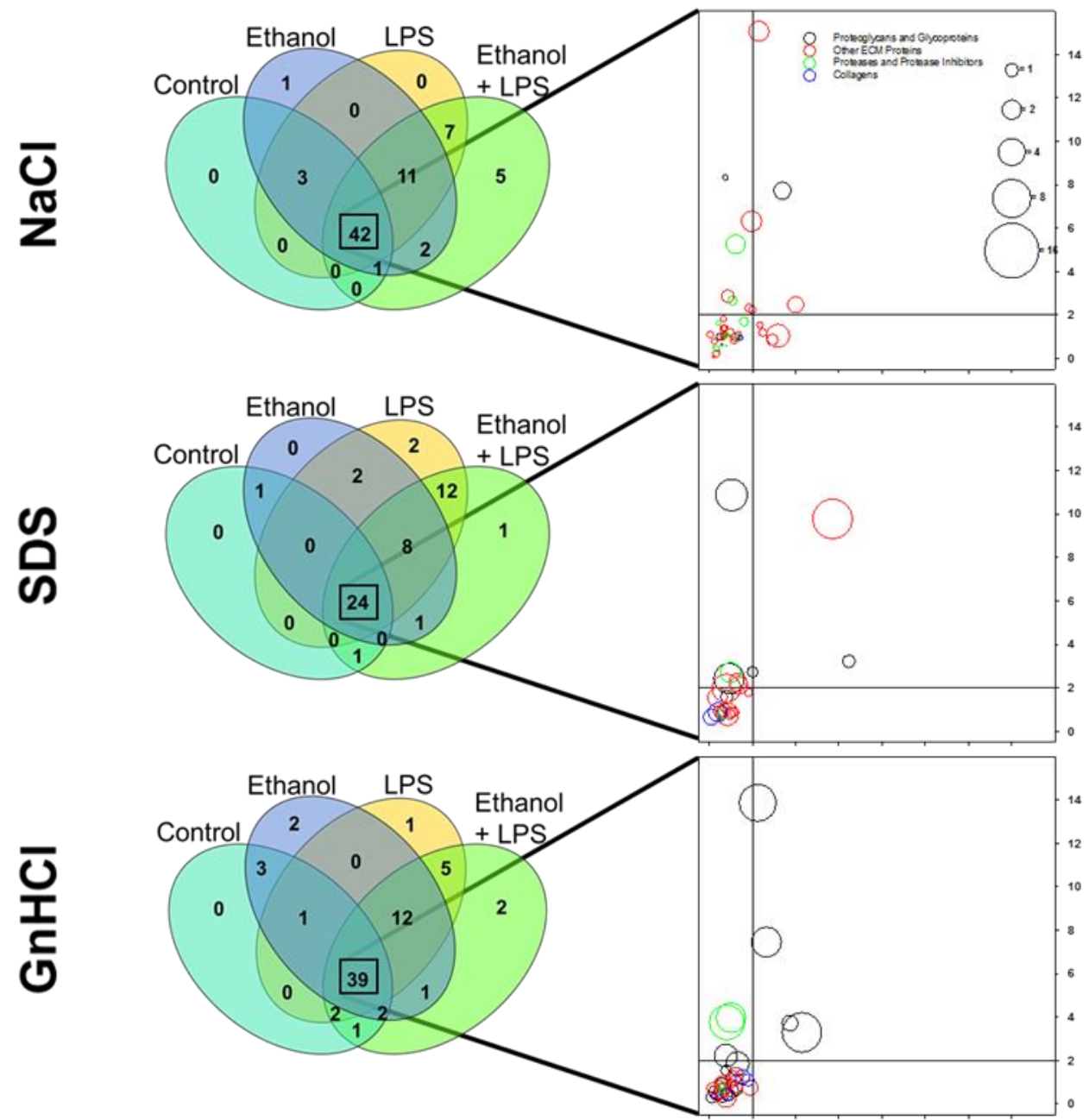

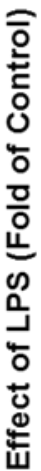

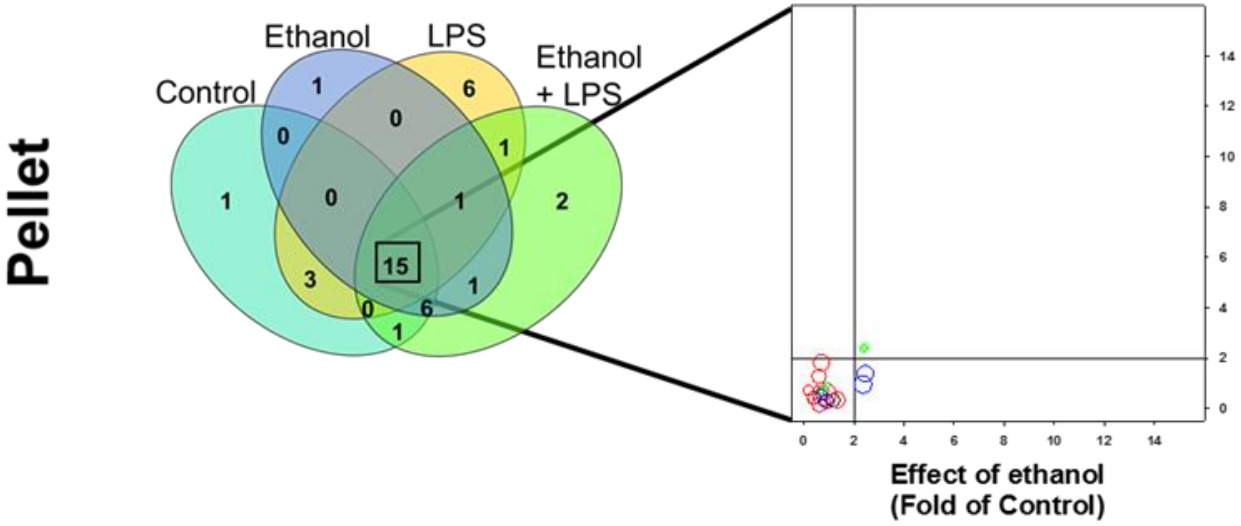


fold) and cysteine-rich with EGF-like domain protein 2 (CREDL2; 6-fold) in the SDS fraction. As was observed for qualitative analysis (Figure 5.3), the pellet fraction was the most stable of the fractions with very few changes in the expression level of ECM proteins.

Some proteins responded to the combination of ethanol and LPS exposure differentially than a simple additive effect. For example, although LPS alone did not change endoplasmin levels and ethanol only increased it 3-fold, the combination of ethanol and LPS increased this protein 11-fold in the $\mathrm{NaCl}$ fraction. A similar effect was seen on granulin expression in the SDS fraction, which was increased by 5 -fold by LPS + ethanol despite no effect by ethanol alone and only a 2-fold increase by LPS. Additionally, although both LPS and ethanol alone increased CRELD2 the combination of ethanol + LPS actually decreased the levels in the SDS fraction. In the $\mathrm{GnHCl}$ fraction, proteins that were differentially regulated by the combination of ethanol and LPS include hemopexin and alpha-1-microglobulin/bikunin precursor (AMPB).

\section{Discussion}

The aim of this work was to develop and characterize a method of hepatic ECM protein extraction and analysis that would provide both the sensitivity to identify low expression proteins and the power to observe global changes in the ECM using proteomics. As mentioned in the Introduction, the study of the hepatic ECM proteome (i.e., matrisome) has largely been 'collagenocentric' and 'fibrisocentric - that is, centered on the dramatic increase in collagen deposition during fibrosis, a quasi-permanent scarring of the organ. However, the 
matrisome of the healthy and diseased liver is significantly more diverse than collagen ECM. Indeed, studies have shown that in addition to collagen, laminin (306) and vitronectin $(309,310)$ are also increased during fibrogenesis.

Furthermore, proteomic-based studies in other organs haves demonstrated that the matrisome responsds dynamically in composition after insult (95-97). Previously, this group showed that fibrin ECM accumulation correlates with inflammatory liver injury in several models, and may well play a causal role (29). Additionally, Gillis et al. have shown a similar role for fibronectin ECM in experimental ALD (28). However, little is known about global changes in the hepatic ECM during inflammatory liver injury.

Global changes in the ECM may mediate tissue function via mechanisms that fall generally into three categories, including physical, biochemical, and signaling. Physical properties of the ECM include the matrix topography, organization, and crosslinking (2). These physical properties support the structural role of the ECM, but can also allow regulation of cell migration both by serving as a physical barriers or enhancer to that function (26). For example, fibrin matrices are permissive to chemotaxis and activation of monocytes and leukocytes $(311,312)$. Physical changes in the ECM can cause tissue rigidity resulting in reduced organ function. Although such physical changes to the ECM can of course indirectly influence the biochemistry of the liver (e.g., hemostasisinduced hypoxia), changes to the mastrisome can also directly cause biochemical changes that are independent of structural changes. For example, ECM components can facilitate interactions between ligands and receptors (313), 
bind and retain chemokines (3), and regulate activation of growth factors (i.e. TGF $\beta$, (3). ECM molecules also can serve directly as signaling molecules via interactions with cell surface receptors, including integrins (314). Because of the multi-faceted roles of many ECM molecules, any single change in the extracellular matrix can trigger a cascade of dependent changes that influence the composition and properties of the ECM. For example, biglycan serves as a structural component which regulates collagen fiber assembly but upon release from the matrix by can serve as a signaling molecule binding to TLR4 receptors (315).

In the current study, the individual and combined effects of two experimental exposures (ethanol and LPS) on the hepatic matrisome were determined. The rationale for selecting LPS is that it induces a robust inflammatory response in the liver. Exposure of the liver to low levels of LPS is common and occurs through multiple means, including both acute and chronic alcohol consumption (316). Whereas inflammatory responses triggered by small doses of LPS are typically non-injurious, other stresses can synergistically enhance the hepatotoxic response to LPS. Indeed, in addition to increasing circulating LPS, ethanol also enhances inflammation and liver damage caused by acute LPS exposure $(71,170)$. This '2-hit' paradigm is a common factor in fatty liver diseases (34). The results here demonstrate that the hepatic matrisome responded dynamically to both stresses, not only increasing the net amount of protein associated with the matrisome, but also differentially changed the 
amounts (Figure 5.5) and likely the composition (Figure 5.3) of the proteins found.

This study was able to validate changes in the hepatic matrisome that have been reported previously after LPS and/or ethanol exposure. Indeed, this work validated that the fibrin(ogen) ECM is dramatically altered by LPS and enhanced by the combination of LPS + ethanol. In the current study, LPS exposure caused a nearly 14 -fold increase in the fibrinogen gamma chain in the $\mathrm{GnHCl}$ extract. Additionally, both LPS and ethanol caused fibrinogen chains to appear in the $\mathrm{NaCl}$ and SDS extracts, suggesting an increased rate of turn-over of this ECM component. The fibrin(ogen) gamma chain is a major component of fibrin clots which form upon cleavage of the fibrinogen gamma chain, causing its polymerization into insoluble fibrin fibers (317). Therefore, the localization of this robust increase in fibrinogen gamma chain in the $\mathrm{GnHCl}$ fractions suggests that there was an increase in fibrin(ogen) gamma chain polymerization into a lesssoluble and more highly cross-linked form. Furthermore, the combination of LPS + ethanol resulted in presence of the fibrin(ogen) gamma chain in the insoluble pellet, which suggests additional modifications that increased insolubility (e.g., crosslinking). LPS also caused the appearance of serum amyloid A-1 and A-2 (SAA1 and SAA2) proteins, which are acute phase proteins whose production and secretion by hepatocytes is increased by LPS $(318,319)$. In fact, LPS administration resulted in the presence of or increased the levels of several other acute phase proteins including haptoglobin, complement $C$, and ceruloplasmin, all of which are known to be increased in liver by LPS administration (320-322). 
Proteomic analysis of liver tissue from animals exposed to chronic ethanol exposure also validated changes that others have shown to be caused by ethanol. Previous studies have shown that ethanol exposure enhances fibronectin expression and deposition prior to the onset of fibrosis (28). In this model of alcoholic steatohepatitis, we found that fibronectin expression was increased by nearly 2 -fold in the $\mathrm{GnHCl}$ extract compared to control livers. Additionally, ethanol exposure increased the level of collagen 1 $1 \alpha 1$, which has previously been shown to be increased in patients (323) and in animal models of alcoholic steatohepatitis (324). Recent studies have also implicated the highmobility group protein B1 as a contributor to ALD and have shown that expression is increased in both the liver and serum after alcohol exposure (325). In our study, HMGB1 was increased in the $\mathrm{NaCl}$ fraction by 2 -fold.

Expression of several other matrix proteins were altered by chronic ethanol exposure. For example, vitronectin levels were increased in the $\mathrm{GnHCl}$ extracts of ethanol-treated liver. The extracellular glycoprotein vitronectin has previously been linked to hepatic fibrosis. Indeed, end-stage hepatic fibrosis is characterized by decreased circulating levels of vitronectin due to decrease in hepatic function and an increase in vitronectin deposition locally in the liver (326, 327). The findings of this study suggest that more subtle changes in vitronectin expression occur prior to the onset of fibrosis and hepatic decompensation. Ethanol also resulted in the appearance of galectin-1. Galectin-1 is glycosaminoglycan-binding lectin (328), which has been associated with cell proliferation and adhesion through modulation of glycoprotein crosslinking. 
Galectin-1 may also play a role in hepatic inflammation and fibrinogenesis (329). For example, galectin-1 has been shown to be protective against experimental inflammatory liver injury (330). This role may be in contrast to later stages of liver disease that correlate increased galectin-1 secretion from activated HSCs in hepatic fibrosis $(331,332)$. Although additional studies will be required to validate functional changes in these proteins after alcohol exposure, these data suggest that ethanol is likely contributing to a myriad of changes in the extracellular matrix composition, many of which have not yet been fully investigated.

Changes in expression of protease and protease inhibitors can also contribute to inflammatory liver injury and fibrogenesis. In the current study, a number of matrix-associated proteases were present in the $\mathrm{GnHCl}$ extract of ethanol-exposed liver including plasmin(ogen), antithrombin III, dipeptidyl peptidase, and alpha-1-antitrypsin. Plasmin(ogen) is a well-known serine protease which degrades fibrin into fibrin degradation products and is tightly regulated by a series of activators (urokinase and tissue-type plasminogen activators) and inhibitors (i.e. plasminogen-activator inhibitor 1; PAI-1). In this study, both ethanol and LPS resulted in the presence of plasminogen in the $\mathrm{GnHCl}$ extract, suggesting that plasmin(ogen) was tightly associated with the ECM after either exposure. LPS also resulted in the presence of protease inhibitors, such as serpine B5 (maspin) and PAI-1. Several other proteases and protease inhibitors that may be critical for ECM homeostasis [e.g., transthyretin, phosphatidylethanolamin-binding protein-1 (PEBP1), and serine protease 
inhibitor $\mathrm{A} 2 \mathrm{~K}$ ] increased in response to ethanol and/or LPS. These data support the notion that transitional remodeling of the hepatic matrisome is likely bidirectional and driven by both increased secretion of matrix proteins as well as changes in ECM degradation.

As mentioned above, ethanol is well-known to synergize liver damage caused by LPS exposure. In the current study, we found that the combination of ethanol and LPS resulted in unique changes to the hepatic matrisome compared to either ethanol or LPS alone. Indeed, fibronectin and biglycan expression were all differentially increase by co-exposure. Fibronectin accumulation caused by ethanol may contribute to hepatic inflammation via stimulation of KCs (308). Biglycan expression was also dramatically increased by the combination of ethanol + LPS. Research suggests that biglycan may play a role in fibrotic liver disease $(315,333)$. This small proteoglycan was first recognized as a structural component and signaling molecule in the ECM due to its association with collagen fibrils and its role in collagen fiber organization $(334,335)$. In addition to its structural role, biglycan has been implicated in inflammation by retaining proinflammatory cytokines including TNF $\alpha$ and TGF $\beta$ (336) and activating TLR4 signaling (337); interestingly, in order to contribute to the inflammatory response, biglycan must be released from the collagens of the ECM by one of several MMPs (338). Areas with increased bigylcan expression have also been associated with increased inflammatory cell migration (339). These data suggest that biglycan expression may be increased in pre-fibrotic stages of liver disease. In contrast, the combination of ethanol and LPS resulted in a synergistic 
decrease in the serine protease inhibitor phosphatidylethanolamine-binding protein-1 (PEBP1). This enzyme has been shown to inhibit trypsin-like serine proteases including thrombin, but not trypsin or tissue-type plasminogen activator (296). Multiple studies have identified PEBP1 as a critical player in metastasis (340), and have defined it as a metastasis suppressor gene (MSG) (341). Additional studies which implicate a role for PEBP1 in inhibition of NFKB (342), a well-recognized player in hepatic inflammation (343) further support a potential role for PEBP1 in inflammatory liver injury. It is likely that other changes in the ECM previously undetermined may also contribute to hepatic inflammation and injury.

In summary, an extraction method to enrich the hepatic ECM was developed and characterized. The results demonstrate that the hepatic matrisome responds dynamically to both acute (LPS) and chronic (ethanol) stresses, long before more dramatic fibrotic changes to the liver. It is likely that these transitional changes to the hepatic ECM contribute, at least in part, to the pathologic responses to these stresses. It is also interesting that several ECM proteins responded similarly to both stresses, suggesting a common mechanism in both models. Nevertheless, there were responses that were unique to the individual and combined effects. These results therefore also serve as a foundation for future analyses in hepatic models of liver disease. 
CHAPTER VI

\section{DISCUSSION AND CONCLUSIONS}

\section{A. Restatement of goals and questions}

The overall goal of the work described in this dissertation was to further elucidate the role of ECM remodeling in liver injury and the potential role of such changes on the pathology of other organs, such as the lung. The experiments in Chapter III were performed to identify the potential role of gut dysbiosis in driving fibrin accumulation and liver injury in a model of enhanced fatty liver disease caused by the combination of HFD and arsenic. Chapter IV aimed to characterize a model of concomitant liver and lung injury in the context of chronic alcohol exposure that will allow further investigation of parallel and interdependent mechanisms of injury in these two organs, including transitional tissue remodeling. Finally, Chapter $V$ of this dissertation describes a new method of protein extraction which can be used for analysis of the extracellular matrix proteome. Together, these studies shed new insight into the complex mechanisms that mediate transitional tissue remodeling and injury in the liver and lung. 


\section{B. Major findings of this dissertation}

\section{Dysbiosis contributes to hepatic sensitization and transitional tissue}

remodeling during NAFLD

Previous studies from this group have shown that coexposure to low concentrations of arsenic and high-fat diet (HFD) enhanced liver injury and inflammation (98); importantly, these effects of arsenic correlated with an increase in proinflammatory fibrin ECM deposition. However, whether enhanced hepatic injury in this model was due to interactions between arsenic and HFD locally in the liver or distally in the gut was unclear. Therefore, the study described in Chapter III was designed to identify changes in the gut microbiome after concomitant HFD and arsenic exposure and to test the therapeutic potential of the prebiotic oligofructose (OFC) in this model; a major endpoint was whether or not this intervention would blunt the increase in fibrin ECM deposition caused by the combination of arsenic and HFD.

Changes in the gut microbiome caused by HFD, as described in Chapter III support the link between HFD and dysbiosis previously demonstrated by others $(78,134)$. Additionally, this study found that arsenic alone impacted the gut microbiome, leading to a net loss of commensal bacteria and expansion of the population of Gram negative bacteria. Increases in Gram negative bacteria, which harbor a lipopolysaccharide wall, can contribute to endotoxemia and have been linked to liver injury $(344,345)$. Therefore, the increase in Gram negative bacteria caused by arsenic may contribute to liver injury by enhancing HFDinduced endotoxemia. Another potential mechanism of enhanced injury may be 
‘upstream' of arsenic exposure. For instance, HFD-induced steatosis and endotoxemia may sensitize the liver to otherwise innocuous levels of arsenic. HFD has previously been shown to sensitize the liver to injury by other factors, including LPS $(145,146)$.

The work presented in Chapter III demonstrated a protective effect of OFC. Protection conferred by OFC was evidenced by a reduction of liver steatosis, fibrin ECM deposition and inflammation that is normally seen with HFD-feeding and a prevention of expansion of Gram negative bacteria caused by arsenic exposure, coupled with an expansion of potentially protective bacterial populations. Protection against enhanced liver injury that was conferred by OFC is likely mediated by both direct effects on the microbiome as well as potentially indirect effects on hepatic sensitization. For example, protection against increases in Gram negative bacteria, which can contribute to endotoxemia, may confer protection against liver injury. Indeed, Adachi and colleagues demonstrated that depletion of Gram negative bacteria by enteral antibiotic administration protected against endotoxemia and liver injury caused by alcohol exposure (344). Additionally, OFC has been shown to protect against HFDinduced endotoxemia (80). In agreement with this previous work, we found that OFC protects against steatosis caused by HFD-feeding to animals. Steatosis and endotoxemia are two factors known to sensitize the liver to injury $(34,35)$. Therefore, protection against these changes by OFC feeding may diminish HFDinduced hepatic sensitization and, ultimately, protect against increased sensitivity to arsenic toxicity. 
It is hypothesized that the enhanced HFD-induced liver injury caused by arsenic exposure is mediated at least in part via deposition of fibrin ECM. This hypothesis is supported by previously established proinflammatory effects of fibrin ECM in liver (29), as well as mechanisms hypothesized to be involved in the atherogenic effects of arsenic in the vasculature (346). Here, this enhanced fibrin accumulation caused by arsenic was almost completely blunted by OFC administration. These data support a role for transitional matrix remodeling in the setting of arsenic hepatotoxicity. Fibrin ECM accumulation in the liver can be mediated via an increase in deposition (via activation of the coagulation cascade), and/or via a decrease in degradation (i.e., fibrinolysis) caused by PAI-1 induction (29). In the work presented here, PAI-1 expression was significantly increased by the combination of arsenic and HFD. The implications of potential crosstalk between transitional tissue remodeling in the setting of fatty liver disease is a focus of future work in this laboratory. The results of the current study lend weight-of-evidence that transitional changes in the hepatic ECM play a unifying role in the initiation and/or progression of fatty liver disease, be it from alcohol or from diet (HFD).

\section{Chronic ethanol exposure causes unique pro-inflammatory changes in the liver and lung after systemic LPS administration}

Chapter IV of this dissertation aimed to characterize a model of concomitant liver and lung injury in the context of chronic ethanol exposure and to establish a potential link between injury of these two organs. As mentioned in the Introduction, work by this group and that of the Roman laboratory has been 
pursuing in parallel the role of transitional matrix changes in inflammatory liver and lung injury. Whereas the detrimental effects of chronic alcohol exposure on the liver and lung are well established separately, potential links between the two are less well characterized. However, clinical studies have suggested a potential interaction between the liver and lung; for example, hepatic cirrhosis increases mortality due to acute lung injury to nearly $100 \%$ (152). Additionally, an experimental model of endotoxic shock has shown that LPS-induced lung damage required interaction with the liver (84). Therefore, the damage to liver and lung caused by transitional ECM changes may not only be parallel pathologic responses, but interdependent mechanisms. Elucidating these mechanisms therefore offers the possibility to develop novel therapeutic approaches. Prior to these critical experiments being performed, the model needed to be developed.

In the work presented here, ethanol preexposure enhanced LPS-induced injury and inflammation in both the liver and the lung. Interestingly, ethanol preexposure caused changes to the mRNA expression of inflammatory cytokines that was unique to each organ. For example, ethanol preexposure caused a significant increase in TNFa mRNA expression in the liver which correlated with a significant increase in plasma levels of TNFa in animals exposed to ethanol and LPS. In contrast, ethanol preexposure did not alter TNFa expression in LPSexposed lungs. Ethanol preexposure did, however, enhance mRNA expression of the TNFa sensitive genes MIP-2 and KC in the lung. Both MIP-2 and KC have been shown to contribute to pulmonary injury caused by LPS $(154,178)$ and 
blocking TNFa expression has been shown to protect against increases MIP-2 and $\mathrm{KC}$ in the lung (88). To address the potential role of TNFa in driving pulmonary MIP-2 levels, we used a TNFa binding antibody, etanercept, in an attempt to block enhanced pulmonary injury caused by ethanol exposure. As expected, etanercept blunted plasma TNFa levels. Etanercept also protected against enhanced pulmonary mRNA expression of MIP-2 and KC in lung and also caused a significant decrease in MIP-2 levels in the BALF. Attenuation of the increase in MIP-2 correlated with protection against pulmonary injury, as indicated by pulmonary pathology. These findings suggest that enhanced systemic TNFa caused by ethanol preexposure may contribute to pulmonary injury. Additionally, Kono and colleagues have suggested that KC-derived inflammatory mediators, including TNF $\alpha$, contribute to pulmonary injury after

systemic LPS exposure (347). Therefore future studies by our lab should aim to elucidate the potential contribution of hepatic-derived TNFa using models that specifically block the release of TNFa from the liver. This work also lays the foundation to investigate the key role of ECM changes in both organs and their independence in pathology.

\section{The hepatic matrisome undergoes dynamic changes after ethanol} and LPS exposure.

The first 2 studies build on our understanding of the role of transitional ECM changes in liver damage (Chapter III) and develop a new model to leverage these questions in the interplay between liver and lung damage (Chapter IV); however, a limitation of previous studies is that they generally investigated 
changes in one ECM protein at a time (e.g., fibrin; Chapter III) and ignore structural changes that may occur with altered ECM protein turnover. Although the role of the ECM is well established in the setting of chronic liver injury and alcoholic liver disease (ALD), the study of the hepatic ECM changes has been focused primarily on dramatic changes in the ECM during fibrogenesis and specifically the contribution of collagen ECM in driving this process. However, recent studies have shown that multiple ECM proteins contribute to fibrogenesis (48), and that the hepatic ECM is altered prior to fibrogenesis (28). Therefore the goal of Chapter $V$ was to adapt a sequential protein extraction method developed for cardiovascular research (95-97) to facilitate LC-MS analysis of global changes in the hepatic matrisome after chronic alcohol exposure. Such an approach could discover new proteins that are changed by insults. Furthermore, the sequential extraction methods yield new insight into potential changes in turnover.

The 3-step protein extraction described in Chapter $V$ allowed efficient extraction of the hepatic ECM proteome into four distinct extracts. These consisted of loosely-associated ECM proteins ( $\mathrm{NaCl}$ extract), intracellular and membrane-associated proteins (SDS extract), tightly bound ECM proteins ( $\mathrm{GnHCl}$ extract), and highly insoluble ECM components (the remaining pellet). A total of 147 extracellular proteins were identified in this study (Table 5.1). Qualitative analysis of the ECM proteins in each fraction demonstrated that both ethanol and LPS caused dynamic changes in the ECM. While neither ethanol nor LPS changed the general pattern of proteins found in the various extracts, 
both tended to increase the number of ECM proteins. Despite no changes in the general pattern of proteins, there were 22 proteins and 14 proteins whose presence was unique to ethanol and LPS exposure, respectively. These unique subsets of proteins may play critical roles in the hepatic response to stress.

There were also quantitative changes in protein abundance caused by ethanol or LPS that may impact function. Interestingly, the combination of ethanol and LPS caused both qualitative and quantitative changes in the hepatic ECM. Analysis of these global changes in the hepatic matrisome may identify new key players in the development of liver injury, the roles of which can be validated in future studies. Furthermore, analysis of changes specific to chronic ethanol exposure may reveal new insights into the mechanisms of liver sensitization caused by alcohol, thus leading to potential therapeutic targets. Most importantly, this work emphasizes dynamism in the hepatic ECM proteome responses to stress that opens a new critical area of future research. This work also lays the foundation for future studies, such as investigating the interactive changes between the hepatic and pulmonary ECM proteome, using the model established in Chapter IV.

\section{Significance of new findings}

The findings of Chapter III have identified dysbiosis as a potential mechanisms by which HFD feeding may predispose individuals to increased toxicity from environmental toxicant, such as arsenic. While the levels of arsenic exposure to most Americans is typically considered sub-toxic, data presented 
here suggest that other factors which alter the gut microbiome, like HFD, may sensitize the liver to injury by otherwise innocuous exposures. Such interactions are becoming more relevant as the incidence of NAFLD continues to increase both nationally as well as globally. Indeed, the estimated prevalence of NAFLD in the US may be as high as $19 \%$ (39), however only a minority of these individuals will progress to later stages of disease (40-42). A better understanding of the interactions that occur between diet and environmental exposures will offer new insights into potential risk factors for the development of NAFLD in the human population and allow better identification of at-risk populations.

The role of inter-organ crosstalk during disease states such as multi-organ failure and sepsis is well established but mechanisms underlying these interactions can be very complex and largely remain incompletely understood. Chapter IV characterized a model of concomitant liver and lung injury in the setting of chronic alcohol exposure. Characterization of this model will bring attention to the parallel mechanisms of injury in these two organs as well as the potential for extensive interdependence between them. Better understanding of the contribution of hepatic-derived mediators in the increased susceptibility to lung injury after alcohol exposure may provide new insights into the mechanisms underlying dysregulated immune response and tissue remodeling in the lung.

Chapter $\mathrm{V}$ describes a new method of hepatic matrisome extraction and analysis. Implementation of this new method will allow identification of new players in fatty liver disease paradigm. Furthermore, adaptation of this method 
will allow analysis of global changes in the extracellular matrix in other models of liver injury (i.e., NAFLD) and in other organs (i.e., the lung). Identifying new targets in disease progression could potentially be beneficial for individuals with early stages of liver disease by offering a means to prevent progression to later stages of disease.

\section{Strengths and Weaknesses}

\section{Strengths}

There are several strengths of this dissertation. One such strength is that it identifies gut dysbiosis as a new potential mechanism mediating interaction between diet-induced liver disease and environmental toxicants (i.e., arsenic exposure). The findings of this study provide additional support for the use of prebiotics as a potential therapeutic for early stages of liver disease, and potentially as a prophylactic therapy to protect against environmental liver disease. This work also describes an initial characterization of a new model of concomitant liver and lung injury after chronic alcohol exposure.

Characterization of this new model will allow a better understanding of how alcohol affects the entire person (or mouse) rather than an individual organ. Use of this model may also provide new insights into why some lung-specific approaches to the study and treatment of pulmonary injury after alcohol exposure are not completely effective and that systemic therapies (e.g., etanercept) may confer new avenues of treatment. 
Additionally, a new method of protein extraction has been validated for use with liver tissue, effectively allowing the analysis of low-expressing and highly insoluble hepatic ECM proteins. LC-MS analysis of these extracts has opened the door for a discovery-based research approach that can help to expand the foundation of new hypothesis-driven studies and possibly increase the identification of new therapeutic targets. Importantly, this work also demonstrates that the hepatic ECM proteome responds dynamically to stress. Whereas such dynamism for the ECM is well understood in other organs and systems, most work in the liver has focused predominantly on hepatic fibrosis.

Another strength of this work is the use of whole animal models. Most of the experiments described here were performed in vivo. As mentioned previously, liver disease and the effects of alcohol use are complex phenomena which are not limited to a single organ or cell type. The use of in vivo models in the experiments described here allowed the study of interactions between multiple organs, including the gut, liver, and lung. Indeed, all of the questions that were addressed here likely could not be sufficiently recapitulated in a simpler model system (e.g., cultured cells).

\section{Weaknesses}

Although the use of animal models of disease is a strength, it is also a potential source of complexity. There is almost always an inverse relationship between the validity of the model and the degree to which the model can be controlled and therefore how certain our conclusions may be. Therefore, 
although in vivo is a more valid approach than simpler models, the 'black box' is also much larger. Furthermore, although in vivo research is preferred for the study of inter-organ phenomena, no animal model can completely recapitulate the human condition. Indeed, there is currently no rodent model that exactly represents the progression of fatty liver disease, increased susceptibility to lung injury, or the typical human microbiome. It is therefore possible that the observed changes in the microbiome or mastrisome are unique to the species tested and have little direct relevance to human beings.

The work presented here identified the microbiome as a site for interaction between a HFD and the environmental contaminant arsenic. This study focused on changes in the microbiome caused by arsenic, HFD and OFC administration rather than the potential effects that the microbiome might be having on these parameters. Lu et al have shown that arsenic-induced dysbiosis does cause functional changes in the gut metabolome (2). Future studies will be necessary to pare out additional, bidirectional changes in the microbiome and arsenic metabolism.

One weakness of this project is the use of systemic administration of etanercept to block TNFa investigate the potential interaction between liver and lung injury. Whereas the liver is known to contribute to enhanced systemic levels of pro-inflammatory cytokines, including TNFa, systemic etanercept binds TNFa, regardless of source. Studies that aim to identify the role of hepatic-derived mediators in the development of lung injury will require an experimental model which allows specific blockage of liver-derived TNFa. 
The experiments described in Chapter $V$ identified changes in the hepatic matrisome after alcohol and LPS exposure. Unfortunately, due to the cost of LCMS analysis, study samples were pooled prior to LC-MS. This is a significant weakness of this study as qualitative changes in protein expression could have been driven by a single sample. Future studies would be strengthened by analysis of individual samples as separate runs, or via 'multiplexing' the samples with protein tagging. Additionally, the experiments described herein did not determine the functional significance of the changes in the ECM. Future studies will be required to validate that changes in the matrisome caused by LPS and/or ethanol cause functional change in vivo and in vitro.

\section{E. Future Directions}

While the experiments described in this dissertation answered specific gaps in our knowledge of ECM remodeling, it has also created new questions that will need to be addressed in future studies. Some of these are discussed below.

\section{Does HFD-induced dysbiosis change arsenic metabolism and/or absorption?}

Because the experiments described in Chapter III were largely concerned with the effect of arsenic, HFD, and OFC on the gut microbiome, the bidirectional effects of dysbiosis on arsenic metabolism and absorption were not determined. Lu and colleagues have previously shown that higher levels of arsenic can cause changes in the gut metabolome (124) and that dysbiosis caused by bacterial infection altered arsenic metabolism (348). In vitro studies have also shown that 
the bacteria of both the mouse and human gut can metabolize arsenic in vitro (141-143). Such findings suggest that dysbiosis caused by HFD could cause dramatic changes in arsenic metabolism. An important endpoint of future studies would include hepatic levels of arsenic and arsenic metabolites. Indeed, increased levels of arsenic in the liver of HFD-fed mice may contribute to enhanced liver injury in the model presented here. On a related note, it would be interesting to determine if arsenic has global effects on the hepatic matrisome; this question is especially valid, since previous work by others has shown that very low concentrations of arsenic cause hepatic endothelial remodeling (46) Work by others in our group are pursuing these questions using the ECM proteomic approach developed in Chapter V.

\section{Does liver-derived TNFa contribute to the alcoholic lung phenotype?}

In the experiments described in Chapter IV, etanercept was used to identify the potential role of extra-pulmonary TNFa in driving lung inflammation. While studies have shown that hepatic Kupffer cells are responsible for $\sim 90 \%$ of the increase in plasma TNF $\alpha$ caused by LPS (172) the role of liver-derived TNF $\alpha$ needs to be determined using a model in which one can specifically block TNFa release from the liver. One approach to this problem would be to use Kupffer cell chimeric mice to impair production of tumor necrosis factor a converting enzyme (TACE) in Kupffer cells. TACE is an enzyme required for cleavage and systemic release of TNFa into the blood. The use of such chimeras would target TACE deficiency to the Kupffer cells and thereby block hepatic TNFa signaling. These 
studies would identify the contribution of hepatic TNFa in the lung alterations caused by chronic alcohol exposure. The protocol for generating the chimeras to perform these studies are currently being optimized.

\section{What are the functional effects of changes in the hepatic matrisome of LPS and ethanol-exposed livers?}

The method described in Chapter $V$ identified dynamic changes in the hepatic matrisome after ethanol and/or LPS exposure. However, the functional effects of these changes has yet to be determined. Others have shown that the extracellular matrix from decellularized tissues can be solubilized and used to coat cell culture plates where it serves as a substratum for cells in vitro (349). By using plates coated with the ECM of animals exposed to ethanol or LPS, the functional effects of changes in the ECM composition on cell migration activation could be determined. In vivo experiments using inhibitors that block the interactions between the ECM and cell surface receptors may also provide insight into the functional effects of changes in the hepatic matrisome after

alcohol and LPS exposure. Indeed, previous studies from our group have shown that the integrin inhibitor CycloRGDfV can protect against enhanced liver injury and inflammation caused by acute ethanol exposure and LPS (350).

\section{What changes does alcohol exposure cause to the lung matrisome?}

Experimental alcohol exposure has been shown to contribute to the alcoholic lung phenotype which is characterized by oxidative stress (58), dysregulated 
immune response $(186,189)$, and tissue remodeling of the lung $(59,351,352)$. Indeed, studies have shown that alcohol exposure increases the expression of fibronectin by pulmonary fibroblasts (68). Additional studies have shown that ethanol also causes increased expression and activity of matrix metalloproteinases (MMPs) (62). Furthermore, the fibronectin-rich matrix produced by alcohol-exposed alveolar macrophages appears to contribute to functional changes in the lung after alcohol exposure, including monocyte activation (353). A discovery-based method, such as that described in Chapter V, would allow for analysis of global changes to the pulmonary matrisome after alcohol exposure. Furthermore, identification of new players in transitional tissue remodeling in the lung would expand the foundation for new hypothesisdriven studies investigating the role of ECM proteins in alcohol-related susceptibility to acute lung injury.

\section{Summary and Conclusions}

The overall goal of the work described in this dissertation was to build upon the unifying hypothesis that changes in the ECM contributes to hepatic pathology as well as pathology in other organs, such as the lung. The experiments described in Chapter III further support the critical role of fibrin deposition in the development of steatohepatitis and the potential contribution of arsenic exposure on hepatic tissue remodeling. These findings suggest that future studies by our group should include investigation of changes in the hepatic extracellular matrix in models of non-alcoholic liver disease. Chapter IV characterized a new model 
of concomitant liver and lung injury in the context of chronic alcohol exposure that will lay the foundation for testing the hypothesis that liver injury is critical to transitional ECM remodeling in the lung. Finally, Chapter $V$ described a new proteomic strategy for characterizing the extracellular proteome which will allow identification of new players in transitional tissue remodeling in the liver and potentially the lung in future experiments. Taken together, this work has shed new insight into the complex interplay between tissue injury and matrix dyshomeostasis. 


\section{REFERENCES}

1. Bosman FT, Stamenkovic I. Functional structure and composition of the extracellular matrix. J Pathol 2003 Jul;200(4):423-428.

2. Lu P, Takai K, Weaver VM, Werb Z. Extracellular matrix degradation and remodeling in development and disease. Cold Spring Harb Perspect Biol $2011 \mathrm{Dec} ; 3(12)$.

3. Hynes RO. The extracellular matrix: not just pretty fibrils. Science 2009 Nov 27;326(5957):1216-1219.

4. Mott JD, Werb Z. Regulation of matrix biology by matrix metalloproteinases. Curr Opin Cell Biol 2004 Oct;16(5):558-564.

5. Page-McCaw A, Ewald AJ, Werb Z. Matrix metalloproteinases and the regulation of tissue remodelling. Nat Rev Mol Cell Biol 2007 Mar;8(3):221233.

6. Streuli C. Extracellular matrix remodelling and cellular differentiation. Curr Opin Cell Biol 1999 Oct;11(5):634-640.

7. Cawston TE, Young DA. Proteinases involved in matrix turnover during cartilage and bone breakdown. Cell Tissue Res 2010 Jan;339(1):221-235.

8. Wang S, Voisin MB, Larbi KY, Dangerfield J, Scheiermann C, Tran M, et al. Venular basement membranes contain specific matrix protein low expression regions that act as exit points for emigrating neutrophils. J Exp Med 2006 Jun 12;203(6):1519-1532.

9. Cahalon L, Hershkoviz R, Gilat D, Miller A, Akiyama SK, Yamada KM, et al. Functional interactions of fibronectin and TNF alpha: a paradigm of physiological linkage between cytokines and extracellular matrix moieties. Cell Adhes Commun 1994 Jul;2(3):269-273.

10. Gailit J, Clark RA. Wound repair in the context of extracellular matrix. Curr Opin Cell Biol 1994 Oct;6(5):717-725.

11. Gilat D, Hershkoviz R, Mekori YA, Vlodavsky I, Lider O. Regulation of adhesion of CD4+ T lymphocytes to intact or heparinase-treated subendothelial extracellular matrix by diffusible or anchored RANTES and MIP-1 beta. J Immunol 1994 Dec 1;153(11):4899-4906. 
12. Woodfin A, Voisin MB, Nourshargh S. Recent developments and complexities in neutrophil transmigration. Curr Opin Hematol 2010 Jan;17(1):9-17.

13. Bataller R, Brenner DA. Liver fibrosis. J Clin Invest 2005 Feb;115(2):209218.

14. Schuppan D, Ruehl M, Somasundaram R, Hahn EG. Matrix as a modulator of hepatic fibrogenesis. Semin Liver Dis 2001 Aug;21(3):351372.

15. Ebihara T, Venkatesan N, Tanaka R, Ludwig MS. Changes in extracellular matrix and tissue viscoelasticity in bleomycin-induced lung fibrosis.

Temporal aspects. Am J Respir Crit Care Med 2000 Oct;162(4 Pt 1):15691576.

16. Meltzer EB, Noble PW. Idiopathic pulmonary fibrosis. Orphanet J Rare Dis 2008;3:8.

17. Rocco PR, Negri EM, Kurtz PM, Vasconcellos FP, Silva GH, Capelozzi $\mathrm{VL}$, et al. Lung tissue mechanics and extracellular matrix remodeling in acute lung injury. Am J Respir Crit Care Med 2001 Sep 15;164(6):10671071.

18. Henriksen PA, Sallenave JM. Human neutrophil elastase: mediator and therapeutic target in atherosclerosis. Int J Biochem Cell Biol 2008;40(67):1095-1100.

19. Kougias $P$, Chai $H$, Lin $P H$, Yao Q, Lumsden AB, Chen C. Defensins and cathelicidins: neutrophil peptides with roles in inflammation, hyperlipidemia and atherosclerosis. J Cell Mol Med 2005 Jan;9(1):3-10.

20. Levental KR, Yu H, Kass L, Lakins JN, Egeblad M, Erler JT, et al. Matrix crosslinking forces tumor progression by enhancing integrin signaling. Cell 2009 Nov 25;139(5):891-906.

21. Provenzano PP, Inman DR, Eliceiri KW, Knittel JG, Yan L, Rueden CT, et al. Collagen density promotes mammary tumor initiation and progression. BMC Med 2008;6:11.

22. Friedman SL, Roll FJ, Boyles J, Bissell DM. Hepatic lipocytes: the principal collagen-producing cells of normal rat liver. Proc Natl Acad Sci U S A 1985 Dec;82(24):8681-8685.

23. Arthur MJ, Iredale JP, Mann DA. Tissue inhibitors of metalloproteinases: role in liver fibrosis and alcoholic liver disease. Alcohol Clin Exp Res 1999 May;23(5):940-943. 
24. Berk BC, Fujiwara K, Lehoux S. ECM remodeling in hypertensive heart disease. J Clin Invest 2007 Mar;117(3):568-575.

25. Liu F, Mih JD, Shea BS, Kho AT, Sharif AS, Tager AM, et al. Feedback amplification of fibrosis through matrix stiffening and COX-2 suppression. J Cell Biol 2010 Aug 23;190(4):693-706.

26. Egeblad M, Rasch MG, Weaver VM. Dynamic interplay between the collagen scaffold and tumor evolution. Curr Opin Cell Biol 2010 Oct;22(5):697-706.

27. Sorokin $L$. The impact of the extracellular matrix on inflammation. Nat Rev Immunol 2010 Oct;10(10):712-723.

28. Gillis SE, Nagy LE. Deposition of cellular fibronectin increases before stellate cell activation in rat liver during ethanol feeding. Alcohol Clin Exp Res 1997 Aug;21(5):857-861.

29. Beier JI, Luyendyk JP, Guo L, von Montfort C, Staunton DE, Arteel GE. Fibrin accumulation plays a critical role in the sensitization to lipopolysaccharide-induced liver injury caused by ethanol in mice. Hepatology 2009 Jan 23;49(5):1545-1553.

30. Altamirano J, Bataller R. Alcoholic liver disease: pathogenesis and new targets for therapy. Nat Rev Gastroenterol Hepatol 2011 Aug 9;8(9):491501.

31. Schwartz JM, Reinus JF. Prevalence and natural history of alcoholic liver disease. Clin Liver Dis 2012 Nov; 16(4):659-666.

32. Seth D, Haber PS, Syn WK, Diehl AM, Day CP. Pathogenesis of alcoholinduced liver disease: classical concepts and recent advances. J Gastroenterol Hepatol 2011 Jul;26(7):1089-1105.

33. Nelson S, Kolls JK. Alcohol, host defence and society. Nature Rev Immunol 2002 Mar;2(3):205-209.

34. Day CP, James OF. Steatohepatitis: a tale of two "hits"? Gastroenterology 1998 Apr;114(4):842-845.

35. Yang SQ, Lin HZ, Lane MD, Clemens M, Diehl AM. Obesity increases sensitivity to endotoxin liver injury: implications for the pathogenesis of steatohepatitis. Proc Natl Acad Sci U S A 1997;94:2557-2562.

36. Berson A, De B, V, Letteron P, Robin MA, Moreau C, El KJ, et al. Steatohepatitis-inducing drugs cause mitochondrial dysfunction and lipid peroxidation in rat hepatocytes. Gastroenterology 1998 Apr;114(4):764774. 
37. Tilg $\mathrm{H}$, Diehl AM. Cytokines in alcoholic and nonalcoholic steatohepatitis. N Engl J Med 2000 Nov 16;343(20):1467-1476.

38. Ogden CL, Carroll MD, Kit BK, Flegal KM. Prevalence of childhood and adult obesity in the United States, 2011-2012. JAMA 2014 Feb 26;311(8):806-814.

39. Lazo M, Hernaez R, Eberhardt MS, Bonekamp S, Kamel I, Guallar E, et al. Prevalence of Nonalcoholic Fatty Liver Disease in the United States: The Third National Health and Nutrition Examination Survey, 1988-1994. Am J Epidemiol 2013 May 27.

40. Angulo P. Nonalcoholic fatty liver disease. N Engl J Med 2002 Apr 18;346(16):1221-1231.

41. Harnois F, Msika S, Sabate JM, Mechler C, Jouet P, Barge J, et al. Prevalence and predictive factors of non-alcoholic steatohepatitis (NASH) in morbidly obese patients undergoing bariatric surgery. Obes Surg 2006 Feb;16(2):183-188.

42. Vernon G, Baranova A, Younossi ZM. Systematic review: the epidemiology and natural history of non-alcoholic fatty liver disease and non-alcoholic steatohepatitis in adults. Aliment Pharmacol Ther 2011 Aug;34(3):274-285.

43. Cave M, Deaciuc I, Mendez C, Song Z, Joshi-Barve S, Barve S, et al. Nonalcoholic fatty liver disease: predisposing factors and the role of nutrition. J Nutr Biochem 2007 Mar;18(3):184-195.

44. Centeno JA, Mullick FG, Martinez L, Page NP, Gibb H, Longfellow D, et al. Pathology related to chronic arsenic exposure. Environ Health Perspect 2002 Oct;110 Suppl 5:883-886.

45. Cowlishaw JL, Pollard EJ, Cowen AE, Powell LW. Liver disease associated with chronic arsenic ingestion. Aust N Z J Med 1979 Jun;9(3):310-313.

46. Straub AC, Stolz DB, Ross MA, Hernandez-Zavala A, Soucy NV, Klei LR, et al. Arsenic stimulates sinusoidal endothelial cell capillarization and vessel remodeling in mouse liver. Hepatology 2007 Jan;45(1):205-212.

47. Arteel GE, Guo L, Schlierf T, Beier JI, Kaiser JP, Chen TS, et al. Subhepatotoxic exposure to arsenic enhances lipopolysaccharide-induced liver injury in mice. Toxicol Appl Pharmacol 2008 Jan 15;226(2):128-139.

48. Gressner OA, Weiskirchen R, Gressner AM. Evolving concepts of liver fibrogenesis provide new diagnostic and therapeutic options. Comp Hepatol 2007;6:7. 
49. Beier JI, Arteel GE. Alcoholic liver disease and the potential role of plasminogen activator inhibitor-1 and fibrin metabolism. Exp Biol Med (Maywood) 2012 Jan 1;237(1):1-9.

50. Bergheim I, Guo L, Davis MA, Lambert JC, Beier JI, Duveau I, et al. Metformin prevents alcohol-induced liver injury in the mouse: Critical role of plasminogen activator inhibitor-1. Gastroenterology 2006 Jun;130(7):2099-2112.

51. Lai KK, Shang S, Lohia N, Booth GC, Masse DJ, Fausto N, et al. Extracellular matrix dynamics in hepatocarcinogenesis: a comparative proteomics study of PDGFC transgenic and Pten null mouse models. PLoS Genet 2011 Jun;7(6):e1002147.

52. Rubenfeld G, Caldwell E, Peabody E, Weaver J, Martin D, Neff M, et al. Incidence and Outcomes of Acute Lung Injury. New Engl J Med 2005;53(3):1685-1693.

53. Moss M, Bucher B, Moore FA, Moore EE, Parsons PE. The role of chronic alcohol abuse in the development of acute respiratory distress syndrome in adults. JAMA 1996 Jan 3;275(1):50-54.

54. Moss M, Burnham EL. Chronic alcohol abuse, acute respiratory distress syndrome, and multiple organ dysfunction. Crit Care Med 2003 Apr;31(4 Suppl):S207-S212.

55. Bechara RI, Brown LA, Eaton DC, Roman J, Guidot DM. Chronic ethanol ingestion increases expression of the angiotensin II type 2 (AT2) receptor and enhances tumor necrosis factor-alpha- and angiotensin II-induced cytotoxicity via AT2 signaling in rat alveolar epithelial cells. Alcohol Clin Exp Res 2003 Jun;27(6):1006-1014.

56. Bechara RI, Brown LA, Roman J, Joshi PC, Guidot DM. Transforming growth factor beta1 expression and activation is increased in the alcoholic rat lung. Am J Respir Crit Care Med 2004 Jul 15;170(2):188-194.

57. Brown LA, Harris FL, Guidot DM. Chronic ethanol ingestion potentiates TNF-alpha-mediated oxidative stress and apoptosis in rat type II cells. Am J Physiol Lung Cell Mol Physiol 2001 Aug;281(2):L377-L386.

58. Holguin F, Moss I, Brown LA, Guidot DM. Chronic ethanol ingestion impairs alveolar type II cell glutathione homeostasis and function and predisposes to endotoxin-mediated acute edematous lung injury in rats. $\mathrm{J}$ Clin Invest 1998 Feb 15;101(4):761-768.

59. Burnham EL, Moss M, Ritzenthaler JD, Roman J. Increased fibronectin expression in lung in the setting of chronic alcohol abuse. Alcohol Clin Exp Res 2007 Apr;31(4):675-683. 
60. Roman J. Extracellular matrix and lung inflammation. Immunol Res 1996;15(2):163-178.

61. D'Souza NB, Baustista AP, Lang CH, Spitzer JJ. Acute ethanol intoxication prevents lipopolysaccharide-induced down regulation of protein kinase C in rat Kupffer cells. Alcohol Clin Exp Res 1992;16:64-67.

62. Lois M, Brown LA, Moss IM, Roman J, Guidot DM. Ethanol ingestion increases activation of matrix metalloproteinases in rat lungs during acute endotoxemia. Am J Respir Crit Care Med 1999 Oct;160(4):1354-1360.

63. Arteel GE. Oxidants and antioxidants in alcohol-induced liver disease. Gastroenterology 2003 Mar;124(3):778-790.

64. Guidot DM, Roman J. Chronic ethanol ingestion increases susceptibility to acute lung injury: role of oxidative stress and tissue remodeling. Chest 2002 Dec;122(6 Suppl):309S-314S.

65. Enomoto N, Ikejima K, Bradford BU, Rivera CA, Kono H, Goto M, et al. Role of Kupffer cells and gut-derived endotoxins in alcoholic liver injury. $J$ Gastroenterol Hepatol 2000 Mar;15 Suppl:D20-D25.

66. Bataller R, Brenner DA. Liver fibrosis. J Clin Invest 2005 Feb;115(2):209218.

67. Dunsmore SE. Treatment of COPD: a matrix perspective. Int J Chron Obstruct Pulmon Dis 2008;3(1):113-122.

68. Roman J, Ritzenthaler JD, Bechara R, Brown LA, Guidot D. Ethanol stimulates the expression of fibronectin in lung fibroblasts via kinasedependent signals that activate CREB. Am J Physiol Lung Cell Mol Physiol 2005 May;288(5):L975-L987.

69. Beier JI, Guo L, von Montfort C, Kaiser JP, Joshi-Barve S, Arteel GE. New role of resistin in lipopolysaccharide-induced liver damage in mice. $\mathrm{J}$ Pharmacol Exp Ther 2008 Mar 13;325(3):801-808.

70. Bergheim I, Guo L, Davis MA, Duveau I, Arteel GE. Critical role of plasminogen activator inhibitor-1 in cholestatic liver injury and fibrosis. $J$ Pharmacol Exp Ther 2006 Feb;316(2):592-600.

71. von Montfort C, Beier JI, Guo L, Kaiser JP, Arteel GE. Contribution of the sympathetic hormone epinephrine to the sensitizing effect of ethanol on LPS-induced liver damage in mice. Am J Physiol Gastrointest Liver Physiol 2008 Mar 6;294(5):G1227-G1234.

72. Eitzman DT, McCoy RD, Zheng X, Fay WP, Shen T, Ginsburg D, et al. Bleomycin-induced pulmonary fibrosis in transgenic mice that either lack 
or overexpress the murine plasminogen activator inhibitor-1 gene. J Clin Invest 1996 Jan 1;97(1):232-237.

73. Zhao Y, Zhong W, Sun X, Song Z, Clemens DL, Kang YJ, et al. Zinc deprivation mediates alcohol-induced hepatocyte IL-8 analog expression in rodents via an epigenetic mechanism. Am J Pathol 2011 Aug;179(2):693-702.

74. Matuschak GM, Rinaldo JE, Pinsky MR, Gavaler JS, Van Thiel DH. Effect of end stage liver failure on the incidence and resolution of adult respiratory distress syndrome. J Crit Care 1987;2:162-173.

75. Abraham E, Matthay M, Dinarello C, Vincent J, Cohen J, Opal S, et al. Consensus conference definitions for sepsis, septic shock, acute lung injury, and acute respirtory distress syndrome: time for a revolution. Crit Care Med 2000;28:232-235.

76. Matuschak GM, Mattingly ME, Tredway TL, Lechner AJ. Liver-lung interactions during E. coli endotoxemia. TNF-alpha:leukotriene axis. Am J Respir Crit Care Med 1994 Jan;149(1):41-49.

77. McClain CJ, Cohen DA. Increased tumor necrosis factor production by monocytes in alcoholic hepatitis. Hepatology 1989;9:349-351.

78. Cani PD, Bibiloni R, Knauf C, Waget A, Neyrinck AM, Delzenne NM, et al. Changes in gut microbiota control metabolic endotoxemia-induced inflammation in high-fat diet-induced obesity and diabetes in mice. Diabetes 2008 Jun;57(6):1470-1481.

79. Brun P, Castagliuolo I, Di Leo V, Buda A, Pinzani M, Palu G, et al. Increased intestinal permeability in obese mice: new evidence in the pathogenesis of nonalcoholic steatohepatitis. 292 ed. 2007. G518-25.

80. Cani PD, Neyrinck AM, Fava F, Knauf C, Burcelin RG, Tuohy KM, et al. Selective increases of bifidobacteria in gut microflora improve high-fatdiet-induced diabetes in mice through a mechanism associated with endotoxaemia. Diabetologia 2007 Nov;50(11):2374-2383.

81. Forsyth CB, Farhadi A, Jakate SM, Tang Y, Shaikh M, Keshavarzian A. Lactobacillus GG treatment ameliorates alcohol-induced intestinal oxidative stress, gut leakiness, and liver injury in a rat model of alcoholic steatohepatitis. Alcohol 2009 Mar;43(2):163-172.

82. Kirpich IA, McClain CJ. Probiotics in the treatment of the liver diseases. J Am Coll Nutr 2012 Feb;31(1):14-23. 
83. Li Z, Yang S, Lin H, Huang J, Watkins PA, Mose AB, et al. Probiotics and antibodies to TNF inhibit inflammatory activity and improve nonalcoholic fatty liver disease. 37 ed. 2003. 343-50.

84. Siore AM, Parker RE, Stecenko AA, Cuppels C, McKean M, Christman $\mathrm{BW}$, et al. Endotoxin-induced acute lung injury requires interaction with the liver. Am J Physiol Lung Cell Mol Physiol 2005 Nov;289(5):L769-L776.

85. Nanji A, Zhoa S, Sadrzadeh S, Waxman D. Use of reverse transcriptionpolymerase chain reaction to evaluate in vivo cytokin gene expression in rats fed ethanol for long periods. Hepatology 1994;21(1304):1309.

86. Hansen J, Cherwitz DL, Allen JI. The role of tumor necrosis factor-alpha in acute endotoxin-induced hepatotoxicity in ethanol-fed rats. Hepatology 1998;20(2):461-474

87. Kamimura S, Tsukamoto H. Cytokine gene expression by Kupffer cells in experimental alcoholic liver disease. Hepatology 1995;21:1304-1309.

88. Saperstein S, Huyck H, Kimball E, Johnston C, Finkelstein J, Pryhuber G. The effects of interleukin-1beta in tumor necrosis factor-alpha-induced acute pulmonary inflammation in mice. Mediators Inflamm 2009;2009:958658.

89. Abraham E, Jesmok G, Tuder R, Allbee J, Chang YH. Contribution of tumor necrosis factor-alpha to pulmonary cytokine expression and lung injury after hemorrhage and resuscitation. Crit Care Med 1995 Aug;23(8):1319-1326.

90. Thiele GM, Duryee MJ, Freeman TL, Sorrell MF, Willis MS, Tuma DJ, et al. Rat sinusoidal liver endothelial cells (SECs) produce pro-fibrotic factors in response to adducts formed from the metabolites of ethanol. Biochem Pharmacol 2005 Nov 25;70(11):1593-1600.

91. Iyer SS, Ramirez AM, Ritzenthaler JD, Torres-Gonzalez E, Roser-Page S, Mora AL, et al. Oxidation of extracellular cysteine/cystine redox state in bleomycin-induced lung fibrosis. Am J Physiol Lung Cell Mol Physiol 2009 Jan;296(1):L37-L45.

92. Kozul CD, Nomikos AP, Hampton TH, Warnke LA, Gosse JA, Davey JC, et al. Laboratory diet profoundly alters gene expression and confounds genomic analysis in mouse liver and lung. Chem Biol Interact 2008 May 28;173(2):129-140.

93. Budinger GR, McKell JL, Urich D, Foiles N, Weiss I, Chiarella SE, et al. Particulate matter-induced lung inflammation increases systemic levels of PAl-1 and activates coagulation through distinct mechanisms. PLoS One 2011;6(4):e18525. 
94. Karabela SP, Kairi CA, Magkouta S, Psallidas I, Moschos C, Stathopoulos I, et al. Neutralization of tumor necrosis factor bioactivity ameliorates urethane-induced pulmonary oncogenesis in mice. Neoplasia 2011 Dec;13(12):1143-1151.

95. Didangelos A, Yin X, Mandal K, Saje A, Smith A, Xu Q, et al. Extracellular matrix composition and remodeling in human abdominal aortic aneurysms: a proteomics approach. Mol Cell Proteomics 2011 Aug;10(8):M111.

96. Didangelos A, Yin X, Mandal K, Baumert M, Jahangiri M, Mayr M. Proteomics characterization of extracellular space components in the human aorta. Mol Cell Proteomics 2010 Sep;9(9):2048-2062.

97. de Castro Bras LE, Ramirez TA, DeLeon-Pennell KY, Chiao YA, Ma Y, Dai Q, et al. Texas 3-step decellularization protocol: looking at the cardiac extracellular matrix. J Proteomics 2013 Jun 28;86:43-52.

98. Tan M, Schmidt RH, Beier JI, Watson WH, Zhong H, States JC, et al. Chronic subhepatotoxic exposure to arsenic enhances hepatic injury caused by high fat diet in mice. Toxicol Appl Pharmacol 2011 Dec 15;257(3):356-364.

99. Bligh EG, Dyer WJ. A rapid method of total lipid extraction and purification. Can J Biochem Physiol 1959;37:911-917.

100. Guo L, Richardson KS, Tucker LM, Doll MA, Hein DW, Arteel GE. Role of the renin-angiotensin system in hepatic ischemia reperfusion injury in rats. Hepatology 2004 Sep;40(3):583-589.

101. Rondanelli M, Sarra S, Antoniello N, Mansi V, Govoni S, Falvo F, et al. No effect of atypical antipsychotic drugs on weight gain and risk of developing type II diabetes or lipid abnormalities among nursing home elderly patients with Alzheimer's disease. Minerva Med 2006 Apr;97(2):147-151.

102. Caporaso JG, Kuczynski J, Stombaugh J, Bittinger K, Bushman FD, Costello EK, et al. QIIME allows analysis of high-throughput community sequencing data. Nat Methods 2010 May;7(5):335-336.

103. DeSantis TZ, Hugenholtz P, Larsen N, Rojas M, Brodie EL, Keller K, et al. Greengenes, a chimera-checked 16S rRNA gene database and workbench compatible with ARB. Appl Environ Microbiol 2006 Jul;72(7):5069-5072.

104. Edgar RC, Haas BJ, Clemente JC, Quince C, Knight R. UCHIME improves sensitivity and speed of chimera detection. Bioinformatics 2011 Aug 15;27(16):2194-2200. 
105. Edgar RC. Search and clustering orders of magnitude faster than BLAST. Bioinformatics 2010 Oct 1;26(19):2460-2461.

106. McDonald D, Price MN, Goodrich J, Nawrocki EP, DeSantis TZ, Probst A, et al. An improved Greengenes taxonomy with explicit ranks for ecological and evolutionary analyses of bacteria and archaea. ISME J 2012 Mar;6(3):610-618.

107. Price MN, Dehal PS, Arkin AP. FastTree 2--approximately maximumlikelihood trees for large alignments. PLoS One 2010;5(3):e9490.

108. Lozupone C, Knight R. UniFrac: a new phylogenetic method for comparing microbial communities. Appl Environ Microbiol 2005 Dec;71(12):82288235.

109. Segata N, Izard J, Waldron L, Gevers D, Miropolsky L, Garrett WS, et al. Metagenomic biomarker discovery and explanation. Genome Biol 2011;12(6):R60.

110. La Rosa PS, Brooks JP, Deych E, Boone EL, Edwards DJ, Wang Q, et al. Hypothesis testing and power calculations for taxonomic-based human microbiome data. PLoS One 2012;7(12):e52078.

111. Dean CB, Nielsen JD. Generalized linear mixed models: a review and some extensions. Lifetime Data Anal 2007 Dec;13(4):497-512.

112. Waalkes MP, Liu J, Chen H, Xie Y, Achanzar WE, Zhou YS, et al. Estrogen signaling in livers of male mice with hepatocellular carcinoma induced by exposure to arsenic in utero. J Natl Cancer Inst 2004 Mar 17;96(6):466-474.

113. Santra A, Das GJ, De BK, Roy B, Guha Mazumder DN. Hepatic manifestations in chronic arsenic toxicity. Indian J Gastroenterol 1999 Oct;18(4):152-155.

114. Mazumder DN. Effect of chronic intake of arsenic-contaminated water on liver. Toxicol Appl Pharmacol 2005 Aug 7;206(2):169-175.

115. Santra A, Maiti A, Das S, Lahiri S, Charkaborty SK, Mazumder DN. Hepatic damage caused by chronic arsenic toxicity in experimental animals. J Toxicol Clin Toxicol 2000;38(4):395-405.

116. Ogden CL, Carroll MD, Kit BK, Flegal KM. Prevalence of obesity in the United States, 2009-2010. NCHS data brief, no 82. National Center for Health Statistics; 2012 Jan. 
117. Fakhouri TH, Ogden CL, Carroll MD, Kit BK, Flegal KM. Prevalence of obesity among older adults in the United States, 2007-2010. 106 ed. 2013. 1-8.

118. Browning JD, Szczepaniak LS, Dobbins R, Nuremberg P, Horton JD, Cohen JC, et al. Prevalence of hepatic steatosis in an urban population in the United States: impact of ethnicity. Hepatology 2004 Dec;40(6):13871395.

119. Bellentani S, Saccoccio G, Masutti F, Croce L, Brandi G, Sasso F, et al. Prevalence of and risk factors for hepatic steatosis in Northern Italy. 132 ed. 2000. 112-117.

120. Clark JM. The epidemiology of nonalcoholic fatty liver disease in adults. J Clin Gastroenterol 2006 Mar;40(3 Suppl 1):S5-10.

121. Choudhry ZK, Misbahuddin M, Hosain AK, Saleh AA. Inhibitory effect of arsenic on aerobic gut flora in rat. Bangladesh Med Res Counc Bull 2009 Dec;35(3):79-83.

122. Li Z, Yang S, Lin H, Huang J, Watkins $P$, Moser $A$, et al. Probiotics and antibodies to TNF inhibit inflammatory activity and improve nonalcoholic fatty liver disease. 37 ed. 2003. 343-350.

123. Kirpich IA, Solovieva NV, Leikhter SN, Shidakova NA, Lebedeva OV, Sidorov PI, et al. Probiotics restore bowel flora and improve liver enzymes in human alcohol-induced liver injury: a pilot study. Alcohol 2008 Dec;42(8):675-682.

124. Lu K, Abo RP, Schlieper KA, Graffam ME, Levine S, Wishnok JS, et al. Arsenic exposure perturbs the gut microbiome and its metabolic profile in mice: an integrated metagenomics and metabolomics analysis. Environ Health Perspect 2014 Mar;122(3):284-291.

125. Ley RE, Backhed F, Turnbaugh P, Lozupone CA, Knight RD, Gordon JI. Obesity alters gut microbial ecology. Proc Natl Acad Sci U S A 2005 Aug 2;102(31):11070-11075.

126. Ferreira RB, Gill N, Willing BP, Antunes LC, Russell SL, Croxen MA, et al. The intestinal microbiota plays a role in Salmonella-induced colitis independent of pathogen colonization. PLoS One 2011;6(5):e20338.

127. Keeney KM, Yurist-Doutsch S, Arrieta MC, Finlay BB. Effects of antibiotics on human microbiota and subsequent disease. Annu Rev Microbiol 2014 Sep 8;68:217-235. 
128. Sokol H, Seksik P, Rigottier-Gois L, Lay C, Lepage P, Podglajen I, et al. Specificities of the fecal microbiota in inflammatory bowel disease. Inflamm Bowel Dis 2006 Feb;12(2):106-111.

129. Wu GD. Diet, the Gut Microbiome and the Metabolome in IBD. Nestle Nutr Inst Workshop Ser 2014;79:73-82.

130. Ochoa-Reparaz J, Mielcarz DW, Ditrio LE, Burroughs AR, Foureau DM, Haque-Begum S, et al. Role of gut commensal microflora in the development of experimental autoimmune encephalomyelitis. J Immunol 2009 Nov 15;183(10):6041-6050.

131. Sellitto M, Bai G, Serena G, Fricke WF, Sturgeon C, Gajer P, et al. Proof of concept of microbiome-metabolome analysis and delayed gluten exposure on celiac disease autoimmunity in genetically at-risk infants. PLoS One 2012;7(3):e33387.

132. West CE. Gut microbiota and allergic disease: new findings. Curr Opin Clin Nutr Metab Care 2014 May;17(3):261-266.

133. Bested AC, Logan AC, Selhub EM. Intestinal microbiota, probiotics and mental health: from Metchnikoff to modern advances: Part I autointoxication revisited. Gut Pathog 2013;5(1):5.

134. Cani PD, Delzenne NM. Involvement of the gut microbiota in the development of low grade inflammation associated with obesity: focus on this neglected partner. Acta Gastroenterol Belg 2010 Apr;73(2):267-269.

135. Ding S, Chi MM, Scull BP, Rigby R, Schwerbrock NM, Magness S, et al. High fat diet: bacteria interactions promote intestinal inflammation which precedes and correlates with obesity and insulin resistance in mosue. 5 ed. 2010. e12191.

136. Rutenburg AM, Sonnenblick E, Koven I, Aprahamian HA, Reiner L, Fine J. The role of intestinal bacteria in the development of dietary cirrhosis in rats. J Exp Med 1957;106:1-14.

137. Adachi Y, Moore LE, Bradford BU, Gao W, Thurman RG. Antibiotics prevent liver injury in rats following long-term exposure to ethanol. Gastroenterology 1995;108:218-224.

138. Broitman SA, Gottlieb LS, Zamcheck N. Influence of neomycin and ingested endotoxin in the pathogenesis of choline deficiency cirrhosis in the adult rat. J Exp Med 1964;119:633-647.

139. LUCKEY TD, REYNIERS JA, GYORGY P, FORBES M. Germfree animals and liver necrosis. Ann N Y Acad Sci 1954 May 10;57(6):932-935. 
140. Sheik CS, Mitchell TW, Rizvi FZ, Rehman Y, Faisal M, Hasnain S, et al. Exposure of soil microbial communities to chromium and arsenic alters their diversity and structure. PLoS One 2012;7(6):e40059.

141. Pinyayev TS, Kohan MJ, Herbin-Davis K, Creed JT, Thomas DJ. Preabsorptive metabolism of sodium arsenate by anaerobic microbiota of mouse cecum forms a variety of methylated and thiolated arsenicals. Chem Res Toxicol 2011 Apr 18;24(4):475-477.

142. Rubin SS DC, Alava P, Zekker I, Du LG, Van de Wiele T. Arsenic thiolation and the role of sulfate-reducing bacteria from the human intestinal tract. Environ Health Perspect 2014 Aug;122(8):817-822.

143. Van de Wiele T, Gallawa CM, Kubachka KM, Creed JT, Basta N, Dayton $\mathrm{EA}$, et al. Arsenic metabolism by human gut microbiota upon in vitro digestion of contaminated soils. Environ Health Perspect 2010 Jul;118(7):1004-1009.

144. Laparra JM, Velez D, Barbera R, Granero L, Polache A, Montoro R, et al. Cytotoxic effect of As(III) in Caco-2 cells and evaluation of its human intestinal permeability. Toxicol In Vitro 2006 Aug;20(5):658-663.

145. Frazier TH, DiBaise JK, McClain CJ. Gut microbiota, intestinal permeability, obesity-induced inflammation, and liver injury. JPEN J Parenter Enteral Nutr 2011 Sep;35(5 Suppl):14S-20S.

146. Harmon RC, Tiniakos DG, Argo CK. Inflammation in nonalcoholic steatohepatitis. Expert Rev Gastroenterol Hepatol 2011 Apr;5(2):189-200.

147. U.S.Department of Health and Human Services. Results from the 2010 National Survey on Drug Use and Health. 2010.

148. World Health Organization. Global status report on alcohol and health. 2011.

149. US Burden of Disease Collaborators. The state of US health, 1990-2010: burden of diseases, injuries, and risk factors. JAMA 2013 Aug 14;310(6):591-608.

150. Brown LA, Harris FL, Ping XD, Gauthier TW. Chronic ethanol ingestion and the risk of acute lung injury: a role for glutathione availability? Alcohol 2004;33:191-197.

151. Khanlou H, Souto H, Lippmann M, Munoz S, Rothstein K, Ozden Z. Resolution of adult respiratory distress syndrome after recovery from fulminant hepatic failure. Am J Med Sci 1999;317:134-136. 
152. Matuschak GM. Lung-liver interactions in sepsis and multiple organ failure syndrome. Clin Chest Med 1996 Mar;17(1):83-98.

153. Bautista AP, Skrepnik N, Niesman MR, Bagby GJ. Elimination of macrophages by liposome-encapsulated dichloromethylene diphosphonate suppresses the endotoxin-induced priming of Kupffer cells. J Leukoc Biol 1994 Mar;55(3):321-327.

154. Calkins CM, Heimbach JK, Bensard DD, Song Y, Raeburn CD, Meng X, et al. TNF receptor I mediates chemokine production and neutrophil accumulation in the lung following systemic lipopolysaccharide. J Surg Res 2001 Dec;101(2):232-237.

155. Lomas-Neira J, Perl M, Venet F, Chung CS, Ayala A. The role and source of tumor necrosis factor-alpha in hemorrhage-induced priming for septic lung injury. Shock 2012 Jun;37(6):611-620.

156. Szabo G, Bala S. Alcoholic liver disease and the gut-liver axis. World J Gastroenterol 2010 Mar 21;16(11):1321-1329.

157. Bjarnason I, Ward K, Peters TJ. The leaky gut of alcoholism: Possible route of entry for toxic compounds. Lancet 1984;1(8370):179-182.

158. Keshavarzian A, Farhadi A, Forsyth CB, Rangan J, Jakate S, Shaikh M, et al. Evidence that chronic alcohol exposure promotes intestinal oxidative stress, intestinal hyperpermeability and endotoxemia prior to development of alcoholic steatohepatitis in rats. J Hepatol 2009;50(3):538-547.

159. Nanji AA, Khettry U, Sadrzadeh SM, Yamanaka T. Severity of liver injury in experimental alcoholic liver disease. Correlation with plasma endotoxin, prostaglandin $E_{2}$, leukotriene $B_{4}$, and thromboxane $B_{2}$. Am J Pathol 1993;142:367-373.

160. Campos CM, Lacerda QN, Marcantonio C, Lauar J, Almeida D, Gamba C, et al. Comparing the effects of acute alcohol consumption in germ-free and conventional mice: the role of the gut microbiota. BMC Microbiol 2014 Sep 16;14(1):240.

161. Nanji AA, Khettry U, Sadrzadeh SMH. Lactobacillus feeding reduces endotoxemia and severity of experimental alcoholic liver disease. Proc Soc Exp Biol Med 1994;205(3):243-247.

162. Bode $\mathrm{C}$, Bode JC. Effect of alcohol consumption on the gut. Best Pract Res Clin Gastroenterol 2003 Aug;17(4):575-592.

163. Lieber CS, Jones DP, DeCarli LM. Effects of prolonged ethanol intake: Production of fatty liver despite adequate diets. J Clin Invest 1965;44:1009-1021. 
164. Barnes MA, McMullen MR, Roychowdhury S, Pisano SG, Liu X, Stavitsky $A B$, et al. Macrophage migration inhibitory factor contributes to ethanolinduced liver injury by mediating cell injury, steatohepatitis, and steatosis. Hepatology 2013 May;57(5):1980-1991.

165. Brandon-Warner E, Schrum LW, Schmidt CM, McKillop IH. Rodent models of alcoholic liver disease: of mice and men. Alcohol 2012 Dec;46(8):715-725.

166. Cohen JI, Roychowdhury S, McMullen MR, Stavitsky AB, Nagy LE. Complement and alcoholic liver disease: role of $\mathrm{C} 1 \mathrm{q}$ in the pathogenesis of ethanol-induced liver injury in mice. Gastroenterology 2010 Aug;139(2):664-74, 674.

167. Dolganiuc A, Petrasek J, Kodys K, Catalano D, Mandrekar P, Velayudham $A$, et al. MicroRNA expression profile in Lieber-DeCarli diet-induced alcoholic and methionine choline deficient diet-induced nonalcoholic steatohepatitis models in mice. Alcohol Clin Exp Res 2009 Oct;33(10):1704-1710.

168. Joshi PC, Applewhite L, Ritzenthaler JD, Roman J, Fernandez AL, Eaton DC, et al. Chronic ethanol ingestion in rats decreases granulocytemacrophage colony-stimulating factor receptor expression and downstream signaling in the alveolar macrophage. J Immunol 2005;175:6837-6845.

169. Velasquez A, Bechara RI, Lewis JF, Malloy J, McCaig L, Brown LA, et al. Glutathione replacement preserves the functional surfactant phospholipid pool size and decreases sepsis-mediated lung dysfunction in ethanol-fed rats. Alcohol Clin Exp Res 2002 Aug;26(8):1245-1251.

170. Enomoto N, Ikejima K, Bradford BU, Rivera CA, Kono H, Brenner DA, et al. Alcohol causes both tolerance and sensitization of rat Kupffer cells via mechanisms dependent on endotoxin. Gastroenterology $1998 \mathrm{Jul}$ 24;115(2):443-451.

171. Mitchell PO, Jensen JS, Ritzenthaler JD, Roman J, Pelaez A, Guidot DM. Alcohol primes the airway for increased interleukin-13 signaling. Alcohol Clin Exp Res 2009;33:505-513.

172. Bautista AP, Skrepnik N, Niesman MR, Bagby GJ. Elimination of macrophages by liposome-encapsulated dichlorovethylene diphosphonate suppresses the endotoxin-induced priming of Kupffer cells. J Leukoc Biol 1994;55:321-327.

173. Ward PA. Role of complement, chemokines, and regulatory cytokines in acute lung injury. Ann N Y Acad Sci 1996 Oct 31;796:104-112. 
174. Abraham E. Neutrophils and acute lung injury. Crit Care Med 2003 Apr;31(4 Suppl):S195-S199.

175. Johnston RA, Mizgerd JP, Shore SA. CXCR2 is essential for maximal neutrophil recruitment and methacholine responsiveness after ozone exposure. Am J Physiol Lung Cell Mol Physiol 2005 Jan;288(1):L61-L67.

176. Lomas-Neira JL, Chung CS, Grutkoski PS, Miller EJ, Ayala A. CXCR2 inhibition suppresses hemorrhage-induced priming for acute lung injury in mice. J Leukoc Biol 2004 Jul;76(1):58-64.

177. Sue RD, Belperio JA, Burdick MD, Murray LA, Xue YY, Dy MC, et al. CXCR2 is critical to hyperoxia-induced lung injury. J Immunol 2004 Mar 15;172(6):3860-3868.

178. Su ZQ, Mo ZZ, Liao JB, Feng XX, Liang YZ, Zhang X, et al. Usnic acid protects LPS-induced acute lung injury in mice through attenuating inflammatory responses and oxidative stress. Int Immunopharmacol 2014 Oct;22(2):371-378.

179. Wang G, Huang X, Li Y, Guo K, Ning P, Zhang Y. PARP-1 inhibitor, DPQ, attenuates LPS-induced acute lung injury through inhibiting NF-kappaBmediated inflammatory response. PLoS One 2013;8(11):e79757.

180. Reutershan J, Morris MA, Burcin TL, Smith DF, Chang D, Saprito MS, et al. Critical role of endothelial CXCR2 in LPS-induced neutrophil migration into the lung. J Clin Invest 2006 Mar;116(3):695-702.

181. Adachi Y, Bradford BU, Gao W, Bojes HK, Thurman RG. Inactivation of Kupffer cells prevents early alcohol-induced liver injury. Hepatology 1994;20:453-460.

182. Jones C, Badger SA, Hoper M, Parks RW, Diamond T, Taylor MA. Hepatic cytokine response can be modulated using the Kupffer cell blocker gadolinium chloride in obstructive jaundice. Int J Surg 2013;11(1):46-51.

183. Kono H, Fujii $\mathrm{H}$, Amemiya $\mathrm{H}$, Asakawa $\mathrm{M}$, Hirai $\mathrm{Y}$, Maki $\mathrm{A}$, et al. Role of Kupffer cells in lung injury in rats administered endotoxin 1. J Surg Res 2005 Dec;129(2):176-189.

184. Pryhuber GS, Huyck HL, Baggs R, Oberdorster G, Finkelstein JN. Induction of chemokines by low-dose intratracheal silica is reduced in TNFR I (p55) null mice. Toxicol Sci 2003 Mar;72(1):150-157.

185. Idell S. Endothelium and disordered fibrin turnover in the injured lung: newly recognized pathways. Crit Care Med 2002 May;30(5 Suppl):S274S280. 
186. Greenberg SS, Xie J, Wang Y, Kolls J, Malinski T, Summer WR, et al. Ethanol suppresses LPS-induced mRNA for nitric oxide synthase II in alveolar macrophages in vivo and in vitro. Alcohol 1994;11:539-547.

187. Boe DM, Nelson S, Zhang P, Quinton L, Bagby GJ. Alcohol-induced suppression of lung chemokine production and the host defense response to Streptococcus pneumoniae. Alcohol Clin Exp Res 2003 Nov;27(11):1838-1845.

188. D'Souza NB, Mandujano JF, Nelson S, Summer WR, Shellito JE. Alcohol ingestion impairs host defenses predisposing otherwise healthy mice to Pneumocystis carinii infection. Alcohol Clin Exp Res 1995 Oct;19(5):12191225.

189. Standiford TJ, Danforth JM. Ethanol feeding inhibits proinflammatory cytokine expression from murine alveolar macrophages ex vivo. Alcohol Clin Exp Res 1997 Oct;21(7):1212-1217.

190. Zhang P, Bagby GJ, Boe DM, Zhong Q, Schwarzenberger P, Kolls JK, et al. Acute alcohol intoxication suppresses the CXC chemokine response during endotoxemia. Alcohol Clin Exp Res 2002 Jan;26(1):65-73.

191. Quinton LJ, Nelson S, Zhang P, Happel KI, Gamble L, Bagby GJ. Effects of systemic and local CXC chemokine administration on the ethanolinduced suppression of pulmonary neutrophil recruitment. Alcohol Clin Exp Res 2005 Jul;29(7):1198-1205.

192. Walker JE, Jr., Odden AR, Jeyaseelan S, Zhang P, Bagby GJ, Nelson S, et al. Ethanol exposure impairs LPS-induced pulmonary LIX expression: alveolar epithelial cell dysfunction as a consequence of acute intoxication. Alcohol Clin Exp Res 2009 Feb;33(2):357-365.

193. Xie J, Kolls J, Bagby G, Greenberg SS. Independent suppression of nitric oxide and TNF alpha in the lung of conscious rats by ethanol. FASEB $\mathrm{J}$ 1995 Feb;9(2):253-261.

194. Zhang P, Nelson S, Summer WR, Spitzer JA. Acute ethanol intoxication suppresses the pulmonary inflammatory response in rats challenged with intrapulmonary endotoxin. Alcohol Clin Exp Res 1997 Aug;21(5):773-778.

195. Liu YD, Liu W, Liu Z. Influence of long-term drinking alcohol on the cytokines in the rats with endogenous and exogenous lung injury. Eur Rev Med Pharmacol Sci 2013 Feb;17(3):403-409.

196. Boe DM, Richens TR, Horstmann SA, Burnham EL, Janssen WJ, Henson $\mathrm{PM}$, et al. Acute and chronic alcohol exposure impair the phagocytosis of apoptotic cells and enhance the pulmonary inflammatory response. Alcohol Clin Exp Res 2010 Oct;34(10):1723-1732. 
197. Friedman SL. Stellate cell activation in alcoholic fibrosis--an overview. Alcohol Clin Exp Res 1999 May;23(5):904-910.

198. Nieto N, Lutolf MP. Extracellular matrix bioengineering and systems biology approaches in liver disease. Syst Synth Biol 2011 Jun;5(1-2):1120.

199. Mason RM, Mayes RW. Extraction of cartilage protein-polysaccharides with inorganic salt solutions. Biochem J 1973 Mar;131(3):535-540.

200. Sajdera SW, Hascall VC. Proteinpolysaccharide complex from bovine nasal cartilage. A comparison of low and high shear extraction procedures. J Biol Chem 1969 Jan 10;244(1):77-87.

201. Fournier T, Medjoubi N, Porquet D. Alpha-1-acid glycoprotein. Biochim Biophys Acta 2000 Oct 18;1482(1-2):157-171.

202. Jahnen-Dechent W, Heiss A, Schafer C, Ketteler M. Fetuin-A regulation of calcified matrix metabolism. Circ Res 2011 Jun 10;108(12):1494-1509.

203. Mitsunaga-Nakatsubo K, Akimoto $Y$, Kusunoki S, Kawakami H. Novel structure of hepatic extracellular matrices containing arylsulfatase $A$. Okajimas Folia Anat Jpn 2013;90(1):17-22.

204. de Groot PG, Meijers JC. beta(2) -Glycoprotein I: evolution, structure and function. J Thromb Haemost 2011 Jul;9(7):1275-1284.

205. Lortat-Jacob H, Burhan I, Scarpellini A, Thomas A, Imberty A, Vives RR, et al. Transglutaminase-2 interaction with heparin: identification of a heparin binding site that regulates cell adhesion to fibronectintransglutaminase-2 matrix. J Biol Chem 2012 May 25;287(22):1800518017.

206. Simon M, Montezin M, Guerrin M, Durieux JJ, Serre G. Characterization and purification of human corneodesmosin, an epidermal basic glycoprotein associated with corneocyte-specific modified desmosomes. J Biol Chem 1997 Dec 12;272(50):31770-31776.

207. Oh-hashi K, Kanamori Y, Hirata Y, Kiuchi K. Characterization of V-ATPase inhibitor-induced secretion of cysteine-rich with EGF-like domains 2. Cell Biol Toxicol 2014 Jun;30(3):127-136.

208. Sofeu Feugaing DD, Gotte M, Viola M. More than matrix: the multifaceted role of decorin in cancer. Eur J Cell Biol 2013 Jan;92(1):1-11.

209. Okamoto O, Fujiwara S. Dermatopontin, a novel player in the biology of the extracellular matrix. Connect Tissue Res 2006;47(4):177-189. 
210. Henry MD, Campbell KP. A role for dystroglycan in basement membrane assembly. Cell 1998 Dec 11;95(6):859-870.

211. Mosesson MW, Siebenlist KR, Meh DA. The structure and biological features of fibrinogen and fibrin. Ann N Y Acad Sci 2001;936:11-30.

212. Mosesson MW. Fibrinogen gamma chain functions. J Thromb Haemost 2003 Feb;1(2):231-238.

213. To WS, Midwood KS. Plasma and cellular fibronectin: distinct and independent functions during tissue repair. Fibrogenesis Tissue Repair 2011;4:21.

214. He J, Baum LG. Galectin interactions with extracellular matrix and effects on cellular function. Methods Enzymol 2006;417:247-256.

215. Sasaki T, Brakebusch C, Engel J, Timpl R. Mac-2 binding protein is a celladhesive protein of the extracellular matrix which self-assembles into ringlike structures and binds beta1 integrins, collagens and fibronectin. EMBO J 1998 Mar 16;17(6):1606-1613.

216. Bateman A, Bennett HP. The granulin gene family: from cancer to dementia. Bioessays 2009 Nov;31(11):1245-1254.

217. de Kleijn DP, Smeets MB, Kemmeren PP, Lim SK, Van Middelaar BJ, Velema $\mathrm{E}$, et al. Acute-phase protein haptoglobin is a cell migration factor involved in arterial restructuring. FASEB J 2002 Jul;16(9):1123-1125.

218. Jones JI, Gockerman A, Busby WH, Jr., Camacho-Hubner C, Clemmons DR. Extracellular matrix contains insulin-like growth factor binding protein5: potentiation of the effects of IGF-I. J Cell Biol 1993 May;121(3):679687.

219. Seltmann K, Roth W, Kroger C, Loschke F, Lederer M, Huttelmaier S, et al. Keratins mediate localization of hemidesmosomes and repress cell motility. J Invest Dermatol 2013 Jan;133(1):181-190.

220. Saito K, Tanaka T, Kanda H, Ebisuno Y, Izawa D, Kawamoto S, et al. Gene expression profiling of mucosal addressin cell adhesion molecule-1+ high endothelial venule cells (HEV) and identification of a leucine-rich HEV glycoprotein as a HEV marker. J Immunol 2002 Feb 1;168(3):1050-1059.

221. Roughley PJ. The structure and function of cartilage proteoglycans. Eur Cell Mater 2006;12:92-101.

222. Penner AS, Rock MJ, Kielty CM, Shipley JM. Microfibril-associated glycoprotein-2 interacts with fibrillin-1 and fibrillin-2 suggesting a role for 
MAGP-2 in elastic fiber assembly. J Biol Chem 2002 Sep 20;277(38):35044-35049.

223. Gao X, Xu Z. Mechanisms of action of angiogenin. Acta Biochim Biophys Sin (Shanghai) $2008 \mathrm{Jul} ; 40(7): 619-624$.

224. Meyer RC, Giddens MM, Coleman BM, Hall RA. The protective role of prosaposin and its receptors in the nervous system. Brain Res 2014 Oct 17;1585C:1-12.

225. Ochiai T, Takenaka Y, Kuramoto Y, Kasuya M, Fukuda K, Kimura M, et al. Molecular mechanism for neuro-protective effect of prosaposin against oxidative stress: its regulation of dimeric transcription factor formation. Biochim Biophys Acta 2008 Dec;1780(12):1441-1447.

226. Elfenbein A, Simons M. Syndecan-4 signaling at a glance. J Cell Sci 2013 Sep 1;126(Pt 17):3799-3804.

227. Li D, Mukai K, Suzuki T, Suzuki R, Yamashita S, Mitani F, et al. Adrenocortical zonation factor 1 is a novel matricellular protein promoting integrin-mediated adhesion of adrenocortical and vascular smooth muscle cells. Febs J 2007 May;274(10):2506-2522.

228. Madsen $C D$, Sidenius $N$. The interaction between urokinase receptor and vitronectin in cell adhesion and signalling. Eur J Cell Biol 2008 Sep;87(89):617-629.

229. Thiel P, Roglin L, Meissner N, Hennig S, Kohlbacher O, Ottmann C. Virtual screening and experimental validation reveal novel small-molecule inhibitors of 14-3-3 protein-protein interactions. Chem Commun (Camb ) 2013 Oct 4;49(76):8468-8470.

230. Dudani AK, Ganz PR. Endothelial cell surface actin serves as a binding site for plasminogen, tissue plasminogen activator and lipoprotein(a). $\mathrm{Br} \mathrm{J}$ Haematol 1996 Oct;95(1):168-178.

231. Mooney DJ, Hansen LK, Langer R, Vacanti JP, Ingber DE. Extracellular matrix controls tubulin monomer levels in hepatocytes by regulating protein turnover. Mol Biol Cell 1994 Dec;5(12):1281-1288.

232. Williams SE, Kounnas MZ, Argraves KM, Argraves WS, Strickland DK. The alpha 2-macroglobulin receptor/low density lipoprotein receptorrelated protein and the receptor-associated protein. An overview. Ann N Y Acad Sci 1994 Sep 10;737:1-13.

233. Roca-Cusachs P, del RA, Puklin-Faucher E, Gauthier NC, Biais N, Sheetz MP. Integrin-dependent force transmission to the extracellular matrix by 
alpha-actinin triggers adhesion maturation. Proc Natl Acad Sci U S A 2013 Apr 9;110(15):E1361-E1370.

234. Burridge K, Nuckolls G, Otey C, Pavalko F, Simon K, Turner C. Actinmembrane interaction in focal adhesions. Cell Differ Dev 1990 Dec 2;32(3):337-342.

235. Vergnolle N, Comera C, Bueno L. Annexin 1 is overexpressed and specifically secreted during experimentally induced colitis in rats. Eur $\mathrm{J}$ Biochem 1995 Sep 1;232(2):603-610.

236. Hajjar KA, Krishnan S. Annexin II: a mediator of the plasmin/plasminogen activator system. Trends Cardiovasc Med 1999 Jul;9(5):128-138.

237. Woods A, Wang G, Beier F. Regulation of chondrocyte differentiation by the actin cytoskeleton and adhesive interactions. J Cell Physiol 2007 Oct;213(1):1-8.

238. Paradis V, Mathurin P, Ratziu V, Poynard T, Bedossa P. Binding of apolipoprotein A-I and acetaldehyde-modified apolipoprotein A-I to liver extracellular matrix. Hepatology 1996 May;23(5):1232-1238.

239. Park JY, Park JH, Jang W, Hwang IK, Kim IJ, Kim HJ, et al. Apolipoprotein A-IV is a novel substrate for matrix metalloproteinases. J Biochem 2012 Mar;151(3):291-298.

240. Hui DY, Basford JE. Distinct signaling mechanisms for apoE inhibition of cell migration and proliferation. Neurobiol Aging 2005 Mar;26(3):317-323.

241. Bernier GM. beta 2-Microglobulin: structure, function and significance. Vox Sang 1980 Jun;38(6):323-327.

242. Gold LI, Eggleton P, Sweetwyne MT, Van Duyn LB, Greives MR, Naylor $\mathrm{SM}$, et al. Calreticulin: non-endoplasmic reticulum functions in physiology and disease. FASEB J 2010 Mar;24(3):665-683.

243. Wang Q, Shen B, Chen L, Zheng P, Feng H, Hao Q, et al. Extracellular calumenin suppresses ERK1/2 signaling and cell migration by protecting fibulin-1 from MMP-13-mediated proteolysis. Oncogene 2014 Mar 17.

244. Imtaiyaz HM, Shajee B, Waheed A, Ahmad F, Sly WS. Structure, function and applications of carbonic anhydrase isozymes. Bioorg Med Chem 2013 Mar 15;21(6):1570-1582.

245. Hellman NE, Gitlin JD. Ceruloplasmin metabolism and function. Annu Rev Nutr 2002;22:439-458. 
246. HogenEsch H, Dunham A, Seymour R, Renninger M, Sundberg JP. Expression of chitinase-like proteins in the skin of chronic proliferative dermatitis (cpdm/cpdm) mice. Exp Dermatol 2006 Oct;15(10):808-814.

247. Yerbury JJ, Poon S, Meehan S, Thompson B, Kumita JR, Dobson CM, et al. The extracellular chaperone clusterin influences amyloid formation and toxicity by interacting with prefibrillar structures. FASEB J 2007 Aug;21(10):2312-2322.

248. Bravo-Cordero JJ, Magalhaes MA, Eddy RJ, Hodgson L, Condeelis J. Functions of cofilin in cell locomotion and invasion. Nat Rev Mol Cell Biol 2013 Jul;14(7):405-415.

249. Suresh MV, Singh SK, Agrawal A. Interaction of calcium-bound C-reactive protein with fibronectin is controlled by $\mathrm{pH}$ : in vivo implications. J Biol Chem 2004 Dec 10;279(50):52552-52557.

250. Sahin H, Borkham-Kamphorst E, Kuppe C, Zaldivar MM, Grouls C, Alsamman $\mathrm{M}$, et al. Chemokine Cxcl9 attenuates liver fibrosis-associated angiogenesis in mice. Hepatology 2012 May;55(5):1610-1619.

251. Romagnani $P$, Lasagni L, Annunziato F, Serio M, Romagnani S. CXC chemokines: the regulatory link between inflammation and angiogenesis. Trends Immunol 2004 Apr;25(4):201-209.

252. Huang $Q Q$, Pope RM. The role of glycoprotein 96 in the persistent inflammation of rheumatoid arthritis. Arch Biochem Biophys 2013 Feb $1 ; 530(1): 1-6$.

253. Manabe R, Tsutsui K, Yamada T, Kimura M, Nakano I, Shimono C, et al. Transcriptome-based systematic identification of extracellular matrix proteins. Proc Natl Acad Sci U S A 2008 Sep 2;105(35):12849-12854.

254. de SM, Smithyman A, Tan C. Suggested models of ecotaxopathy in lymphoreticular malignancy. A role for iron-binding proteins in the control of lymphoid cell migration. Am J Pathol 1978 Feb;90(2):497-520.

255. Kho DH, Zhang T, Balan V, Wang Y, Ha SW, Xie Y, et al. Autocrine motility factor modulates EGF-mediated invasion signaling. Cancer Res 2014 Apr 15;74(8):2229-2237.

256. Everett AD, Lobe DR, Matsumura ME, Nakamura H, McNamara CA. Hepatoma-derived growth factor stimulates smooth muscle cell growth and is expressed in vascular development. J Clin Invest 2000 Mar;105(5):567-575. 
257. Huttunen HJ, Rauvala $\mathrm{H}$. Amphoterin as an extracellular regulator of cell motility: from discovery to disease. J Intern Med 2004 Mar;255(3):351366.

258. Radisky DC, Stallings-Mann M, Hirai Y, Bissell MJ. Single proteins might have dual but related functions in intracellular and extracellular microenvironments. Nat Rev Mol Cell Biol 2009 Mar;10(3):228-234.

259. Neisch AL, Fehon RG. Ezrin, Radixin and Moesin: key regulators of membrane-cortex interactions and signaling. Curr Opin Cell Biol 2011 Aug;23(4):377-382.

260. Huang Y, Arora P, McCulloch CA, Vogel WF. The collagen receptor DDR1 regulates cell spreading and motility by associating with myosin IIA. J Cell Sci 2009 May 15;122(Pt 10):1637-1646.

261. Yan L, Borregaard N, Kjeldsen L, Moses MA. The high molecular weight urinary matrix metalloproteinase (MMP) activity is a complex of gelatinase B/MMP-9 and neutrophil gelatinase-associated lipocalin (NGAL). Modulation of MMP-9 activity by NGAL. J Biol Chem 2001 Oct 5;276(40):37258-37265.

262. Petersson U, Somogyi E, Reinholt FP, Karlsson T, Sugars RV, Wendel M. Nucleobindin is produced by bone cells and secreted into the osteoid, with a potential role as a modulator of matrix maturation. Bone 2004 Jun;34(6):949-960.

263. Peng H, Vijayakumar S, Schiene-Fischer C, Li H, Purkerson JM, Malesevic M, et al. Secreted cyclophilin A, a peptidylprolyl cis-trans isomerase, mediates matrix assembly of hensin, a protein implicated in epithelial differentiation. J Biol Chem 2009 Mar 6;284(10):6465-6475.

264. Fonovic $M$, Turk B. Cysteine cathepsins and extracellular matrix degradation. Biochim Biophys Acta 2014 Aug;1840(8):2560-2570.

265. Zimmer DB, Eubanks JO, Ramakrishnan D, Criscitiello MF. Evolution of the S100 family of calcium sensor proteins. Cell Calcium 2013 Mar;53(3):170-179.

266. Preciado-Patt L, Hershkoviz R, Fridkin M, Lider O. Serum amyloid A binds specific extracellular matrix glycoproteins and induces the adhesion of resting CD4+ T cells. J Immunol 1996 Feb 1;156(3):1189-1195.

267. Zahedi K. Characterization of the binding of serum amyloid $P$ to type IV collagen. J Biol Chem 1996 Jun 21;271(25):14897-14902.

268. Zahedi K. Characterization of the binding of serum amyloid $\mathrm{P}$ to laminin. $\mathrm{J}$ Biol Chem 1997 Jan 24;272(4):2143-2148. 
269. Nozik-Grayck E, Suliman HB, Piantadosi CA. Extracellular superoxide dismutase. Int J Biochem Cell Biol 2005 Dec;37(12):2466-2471.

270. Yi HS, Jeong WI. Interaction of hepatic stellate cells with diverse types of immune cells: foe or friend? J Gastroenterol Hepatol 2013 Aug;28 Suppl 1:99-104.

271. Schoumacher M, Goldman RD, Louvard D, Vignjevic DM. Actin, microtubules, and vimentin intermediate filaments cooperate for elongation of invadopodia. J Cell Biol 2010 May 3;189(3):541-556.

272. Chun RF, Peercy BE, Orwoll ES, Nielson CM, Adams JS, Hewison M. Vitamin D and DBP: the free hormone hypothesis revisited. J Steroid Biochem Mol Biol 2014 Oct;144 Pt A:132-137.

273. Janciauskiene S. Conformational properties of serine proteinase inhibitors (serpins) confer multiple pathophysiological roles. Biochim Biophys Acta 2001 Mar 26;1535(3):221-235.

274. Akerstrom B, Logdberg L, Berggard T, Osmark P, Lindqvist A. alpha(1)Microglobulin: a yellow-brown lipocalin. Biochim Biophys Acta 2000 Oct 18;1482(1-2):172-184.

275. Fries $\mathrm{E}$, Blom AM. Bikunin--not just a plasma proteinase inhibitor. Int $\mathrm{J}$ Biochem Cell Biol 2000 Feb;32(2):125-137.

276. Machovich R, Horvath I. Heparin-like effect of polymethacrylic acid on the reaction between thrombin and antithrombin-III. Thromb Res 1977 Dec;11(6):765-772.

277. Luan Y, Kong L, Howell DR, Ilalov K, Fajardo M, Bai XH, et al. Inhibition of ADAMTS-7 and ADAMTS-12 degradation of cartilage oligomeric matrix protein by alpha-2-macroglobulin. Osteoarthritis Cartilage 2008 Nov;16(11):1413-1420.

278. Mourey L, Samama JP, Delarue M, Choay J, Lormeau JC, Petitou M, et al. Antithrombin III: structural and functional aspects. Biochimie 1990 Aug;72(8):599-608.

279. White $\mathrm{JH}$. Vitamin $\mathrm{D}$ as an inducer of cathelicidin antimicrobial peptide expression: past, present and future. J Steroid Biochem Mol Biol 2010 Jul;121(1-2):234-238.

280. Skrzydlewska E, Sulkowska M, Koda M, Sulkowski S. Proteolyticantiproteolytic balance and its regulation in carcinogenesis. World $\mathrm{J}$ Gastroenterol 2005 Mar 7;11(9):1251-1266. 
281. Santamaria I, Velasco G, Pendas AM, Fueyo A, Lopez-Otin C. Cathepsin $Z$, a novel human cysteine proteinase with a short propeptide domain and a unique chromosomal location. J Biol Chem 1998 Jul 3;273(27):1681616823.

282. Akkari L, Gocheva V, Kester JC, Hunter KE, Quick ML, Sevenich L, et al. Distinct functions of macrophage-derived and cancer cell-derived cathepsin Z combine to promote tumor malignancy via interactions with the extracellular matrix. Genes Dev 2014 Oct 1;28(19):2134-2150.

283. Walport MJ. Complement. First of two parts. N Engl J Med 2001 Apr 5;344(14):1058-1066.

284. Saito F, Saito-Arai Y, Nakamura A, Shimizu T, Matsumura K. Processing and secretion of the N-terminal domain of alpha-dystroglycan in cell culture media. FEBS Lett 2008 Feb 6;582(3):439-444.

285. Nemoto E, Gotoh K, Tsuchiya M, Sakisaka Y, Shimauchi H. Extracellular ATP inhibits IL-1-induced MMP-1 expression through the action of CD39/nucleotidase triphosphate dephosphorylase-1 on human gingival fibroblasts. Int Immunopharmacol 2013 Nov;17(3):513-518.

286. Dietzel E, Wessling J, Floehr J, Schafer C, Ensslen S, Denecke B, et al. Fetuin-B, a liver-derived plasma protein is essential for fertilization. Dev Cell 2013 Apr 15;25(1):106-112.

287. Olivier E, Soury E, Ruminy P, Husson A, Parmentier F, Daveau M, et al. Fetuin-B, a second member of the fetuin family in mammals. Biochem $\mathrm{J}$ 2000 Sep 1;350 Pt 2:589-597.

288. Nag S, Larsson M, Robinson RC, Burtnick LD. Gelsolin: the tail of a molecular gymnast. Cytoskeleton (Hoboken ) 2013 Jul;70(7):360-384.

289. Bakker WW, Spaans F, el BL, Borghuis $T$, van GH, van DE, et al. Plasma hemopexin as a potential regulator of vascular responsiveness to angiotensin II. Reprod Sci 2013 Mar;20(3):234-237.

290. Authier F, Posner BI, Bergeron JJ. Insulin-degrading enzyme. Clin Invest Med 1996 Jun;19(3):149-160.

291. Chen L, Mao SJ, McLean LR, Powers RW, Larsen WJ. Proteins of the inter-alpha-trypsin inhibitor family stabilize the cumulus extracellular matrix through their direct binding with hyaluronic acid. J Biol Chem 1994 Nov 11;269(45):28282-28287.

292. Nakimbugwe D, Masschalck B, Atanassova M, Zewdie-Bosuner A, Michiels CW. Comparison of bactericidal activity of six lysozymes at 
atmospheric pressure and under high hydrostatic pressure. Int J Food Microbiol 2006 May 1;108(3):355-363.

293. Overbergh L, Torrekens S, Van LF, Van den Berghe H. Molecular characterization of the murinoglobulins. J Biol Chem 1991 Sep 5;266(25):16903-16910.

294. Overbergh L, Hilliker C, Lorent K, Van LF, Van den Berghe H. Identification of four genes coding for isoforms of murinoglobulin, the monomeric mouse alpha 2-macroglobulin: characterization of the exons coding for the bait region. Genomics 1994 Aug;22(3):530-539.

295. Xie D, Liu Z, Li Z, Ji Y, Chen J, Sun B. Differential expression of neutrophilic granule proteins between Th1 and Th2 cells. Acta Biochim Biophys Sin (Shanghai) 2007 Jan;39(1):67-72.

296. Hengst U, Albrecht H, Hess D, Monard D. The phosphatidylethanolaminebinding protein is the prototype of a novel family of serine protease inhibitors. J Biol Chem 2001 Jan 5;276(1):535-540.

297. Andreasen PA, Egelund R, Petersen $\mathrm{HH}$. The plasminogen activation system in tumor growth, invasion, and metastasis. Cell Mol Life Sci 2000 Jan 20;57(1):25-40.

298. Kaiserman D, Bird PI. Control of granzymes by serpins. Cell Death Differ 2010 Apr;17(4):586-595.

299. van GD, Sharp P, Morgan K, Kalsheker N. Serpins: structure, function and molecular evolution. Int J Biochem Cell Biol 2003 Nov;35(11):1536-1547.

300. Jin X, Stamnaes J, Klock C, DiRaimondo TR, Sollid LM, Khosla C. Activation of extracellular transglutaminase 2 by thioredoxin. $\mathrm{J}$ Biol Chem 2011 Oct 28;286(43):37866-37873.

301. Palha JA. Transthyretin as a thyroid hormone carrier: function revisited. Clin Chem Lab Med 2002 Dec;40(12):1292-1300.

302. Liz MA, Faro CJ, Saraiva MJ, Sousa MM. Transthyretin, a new cryptic protease. J Biol Chem 2004 May 14;279(20):21431-21438.

303. Ricard-Blum S. The collagen family. Cold Spring Harb Perspect Biol 2011 Jan;3(1):a004978.

304. Zhang Z, Wang F, Wang BJ, Chu G, Cao Q, Sun BG, et al. Inhibition of leptin-induced vascular extracellular matrix remodelling by adiponectin. $\mathrm{J}$ Mol Endocrinol 2014 Oct;53(2):145-154. 
305. Takahashi K, Ip WE, Michelow IC, Ezekowitz RA. The mannose-binding lectin: a prototypic pattern recognition molecule. Curr Opin Immunol 2006 Feb;18(1):16-23.

306. Eyre DR, Paz MA, Gallop PM. Cross-linking in collagen and elastin. Annu Rev Biochem 1984;53:717-748.

307. Tsukamoto H, Takei Y, McClain CJ, Joshi-Barve S, Hill D, Schmidt J, et al. How Is the Liver Primed or Sensitized for Alcoholic Liver Disease? Alcohol Clin Exp Res 2001;25(5):171S-181S.

308. Aziz-Seible RS, Lee SM, Kharbanda KK, McVicker BL, Casey CA. Ethanol feeding potentiates the pro-inflammatory response of Kupffer cells to cellular fibronectin. Alcohol Clin Exp Res 2011 Apr;35(4):717-725.

309. Kobayashi J, Yamada S, Kawasaki H. Distribution of vitronectin in plasma and liver tissue: relationship to chronic liver disease. Hepatology 1994 Dec;20(6):1412-1417.

310. Koukoulis GK, Shen J, Virtanen I, Gould VE. Vitronectin in the cirrhotic liver: an immunomarker of mature fibrosis. Hum Pathol 2001 Dec;32(12):1356-1362.

311. Holdsworth SR, Thomson NM, Glasgow EF, Atkins RC. The effect of defibrination on macrophage participation in rabbit nephrotoxic nephritis: studies using glomerular culture and electronmicroscopy. Clin Exp Immunol 1979 Jul;37(1):38-43.

312. Loike JD, el Khoury J, Cao L, Richards CP, Rascoff H, Mandeville JT, et al. Fibrin regulates neutrophil migration in response to interleukin 8 , leukotriene B4, tumor necrosis factor, and formyl-methionyl-leucylphenylalanine. J Exp Med 1995 May 1;181(5):1763-1772.

313. Lonai $P$. Epithelial mesenchymal interactions, the ECM and limb development. J Anat 2003 Jan;202(1):43-50.

314. Hynes RO. Integrins: versatility, modulation, and signaling in cell adhesion. Cell 1992 Apr 3;69(1):11-25.

315. Nastase MV, Young MF, Schaefer L. Biglycan: a multivalent proteoglycan providing structure and signals. J Histochem Cytochem 2012 Dec;60(12):963-975.

316. Bode $\mathrm{CH}$, Kugler $\mathrm{V}$, Bode $\mathrm{JCH}$. Endotoxemia in patients with alcoholic and non-alcoholic cirrhosis and in subjects with no evidence of chronic liver disease following acute alcohol excess. J Hepatol 1987;4:8-14. 
317. Mosesson MW. Fibrinogen gamma chain functions. J Thromb Haemost 2003 Feb;1(2):231-238.

318. Migita K, Abiru S, Nakamura M, Komori A, Yoshida Y, Yokoyama T, et al. Lipopolysaccharide signaling induces serum amyloid $A(S A A)$ synthesis in human hepatocytes in vitro. FEBS Lett 2004 Jul 2;569(1-3):235-239.

319. Pruett BS, Pruett SB. An explanation for the paradoxical induction and suppression of an acute phase response by ethanol. Alcohol 2006 Jun;39(2):105-110.

320. Bopst M, Haas C, Car B, Eugster HP. The combined inactivation of tumor necrosis factor and interleukin-6 prevents induction of the major acute phase proteins by endotoxin. Eur J Immunol 1998 Dec;28(12):4130-4137.

321. Haziot $A$, Lin $X Y$, Zhang F, Goyert SM. The induction of acute phase proteins by lipopolysaccharide uses a novel pathway that is CD14independent. J Immunol 1998 Mar 15;160(6):2570-2572.

322. Sun S, Guo Y, Zhao G, Zhou X, Li J, Hu J, et al. Complement and the alternative pathway play an important role in LPS/D-GalN-induced fulminant hepatic failure. PLoS One 2011;6(11):e26838.

323. Tiniakos DG. Liver biopsy in alcoholic and non-alcoholic steatohepatitis patients. Gastroenterol Clin Biol 2009 Oct;33(10-11):930-939.

324. Lieber CS, Leo MA, Mak KM, Xu Y, Cao Q, Ren C, et al. Model of nonalcoholic steatohepatitis. Am J Clin Nutr 2004 Mar;79(3):502-509.

325. Ge X, Antoine DJ, Lu Y, Arriazu E, Leung TM, Klepper AL, et al. High mobility group box-1 (HMGB1) participates in the pathogenesis of alcoholic liver disease (ALD). J Biol Chem 2014 Aug 15;289(33):2267222691.

326. Kobayashi J, Yamada S, Kawasaki H. Distribution of vitronectin in plasma and liver tissue: relationship to chronic liver disease. Hepatology 1994 Dec;20(6):1412-1417.

327. Koukoulis GK, Shen J, Virtanen I, Gould VE. Vitronectin in the cirrhotic liver: an immunomarker of mature fibrosis. Hum Pathol 2001 Dec;32(12):1356-1362.

328. Barondes SH, Cooper DN, Gitt MA, Leffler H. Galectins. Structure and function of a large family of animal lectins. J Biol Chem 1994 Aug 19;269(33):20807-20810.

329. Bacigalupo ML, Manzi M, Rabinovich GA, Troncoso MF. Hierarchical and selective roles of galectins in hepatocarcinogenesis, liver fibrosis and 
inflammation of hepatocellular carcinoma. World J Gastroenterol 2013 Dec 21;19(47):8831-8849.

330. Santucci L, Fiorucci S, Cammilleri F, Servillo G, Federici B, Morelli A. Galectin-1 exerts immunomodulatory and protective effects on concanavalin A-induced hepatitis in mice. Hepatology 2000 Feb;31(2):399-406.

331. Kristensen DB, Kawada N, Imamura K, Miyamoto Y, Tateno C, Seki S, et al. Proteome analysis of rat hepatic stellate cells. Hepatology 2000 Aug;32(2):268-277.

332. Maeda N, Kawada N, Seki S, Arakawa T, Ikeda K, Iwao H, et al. Stimulation of proliferation of rat hepatic stellate cells by galectin-1 and galectin-3 through different intracellular signaling pathways. J Biol Chem 2003 May 23;278(21):18938-18944.

333. Hogemann B, Edel G, Schwarz K, Krech R, Kresse H. Expression of biglycan, decorin and proteoglycan-100/CSF-1 in normal and fibrotic human liver. Pathol Res Pract 1997;193(11-12):747-751.

334. Schonherr E, Witsch-Prehm P, Harrach B, Robenek H, Rauterberg J, Kresse H. Interaction of biglycan with type I collagen. J Biol Chem 1995 Feb 10;270(6):2776-2783.

335. Zhang G, Chen S, Goldoni S, Calder BW, Simpson HC, Owens RT, et al. Genetic evidence for the coordinated regulation of collagen fibrillogenesis in the cornea by decorin and biglycan. J Biol Chem 2009 Mar 27;284(13):8888-8897.

336. Tufvesson E, Westergren-Thorsson G. Tumour necrosis factor-alpha interacts with biglycan and decorin. FEBS Lett 2002 Oct 23;530(1-3):124128.

337. Schaefer L, Babelova A, Kiss E, Hausser HJ, Baliova M, Krzyzankova M, et al. The matrix component biglycan is proinflammatory and signals through Toll-like receptors 4 and 2 in macrophages. J Clin Invest 2005 Aug;115(8):2223-2233.

338. Frey H, Schroeder N, Manon-Jensen T, lozzo RV, Schaefer L. Biological interplay between proteoglycans and their innate immune receptors in inflammation. Febs J 2013 May;280(10):2165-2179.

339. Mohan H, Krumbholz M, Sharma R, Eisele S, Junker A, Sixt M, et al. Extracellular matrix in multiple sclerosis lesions: Fibrillar collagens, biglycan and decorin are upregulated and associated with infiltrating immune cells. Brain Pathol 2010 Sep;20(5):966-975. 
340. Xu YF, Yi Y, Qiu SJ, Gao Q, Li YW, Dai CX, et al. PEBP1 downregulation is associated to poor prognosis in $\mathrm{HCC}$ related to hepatitis $\mathrm{B}$ infection. $\mathrm{J}$ Hepatol 2010 Nov;53(5):872-879.

341. Keller ET, Fu Z, Brennan M. The biology of a prostate cancer metastasis suppressor protein: Raf kinase inhibitor protein. J Cell Biochem 2005 Feb 1;94(2):273-278.

342. Tang H, Park S, Sun SC, Trumbly R, Ren G, Tsung E, et al. RKIP inhibits NF-kappaB in cancer cells by regulating upstream signaling components of the IkappaB kinase complex. FEBS Lett 2010 Feb 19;584(4):662-668.

343. Schwabe RF, Brenner DA. Mechanisms of Liver Injury. I. TNF-alphainduced liver injury: role of IKK, JNK, and ROS pathways. Am J Physiol Gastrointest Liver Physiol 2006 Apr;290(4):G583-G589.

344. Adachi Y, Moore LE, Bradford BU, Gao W, Arii S, Imamura M, et al. Antibiotics prevent liver injury following chronic exposure of rats to ethanol. In: Wisse E, Knook DL, Wake K, eds. Cells of the Hepatic Sinusoid.Leiden, The Netherlands: The Kupffer Cell Foundation, 1995. 7274.

345. Nanji AA, Khettry U, Sadrzadeh SMH. Lactobacillus feeding reduces endotoxemia and severity of experimental alcoholic liver disease. Proc Soc Exp Biol Med 1994;205(3):243-247.

346. Srivastava S, Vladykovskaya EN, Haberzettl P, Sithu SD, D'Souza SE, States JC. Arsenic exacerbates atherosclerotic lesion formation and inflammation in ApoE-/- mice. Toxicol Appl Pharmacol 2009 Nov 15;241(1):90-100.

347. Chen CH, Budas GR, Churchill EN, Disatnik MH, Hurley TD, MochlyRosen D. Activation of aldehyde dehydrogenase-2 reduces ischemic damage to the heart. Science 2008 Sep 12;321(5895):1493-1495.

348. Lu K, Cable PH, Abo RP, Ru H, Graffam ME, Schlieper KA, et al. Gut microbiome perturbations induced by bacterial infection affect arsenic biotransformation. Chem Res Toxicol 2013 Dec 16;26(12):1893-1903.

349. Lee JS, Shin J, Park HM, Kim YG, Kim BG, Oh JW, et al. Liver extracellular matrix providing dual functions of two-dimensional substrate coating and three-dimensional injectable hydrogel platform for liver tissue engineering. Biomacromolecules 2014 Jan 13;15(1):206-218.

350. Massey VL, Arteel GE. Acute alcohol-induced liver injury. Frontiers in Physiology 2012;3:1-8. 
351. Shin OS, Isberg RR, Akira S, Uematsu S, Behera AK, Hu LT. Distinct roles for MyD88 and Toll-like receptors 2, 5, and 9 in phagocytosis of Borrelia burgdorferi and cytokine induction. Infect Immun 2008 Jun;76(6):2341-2351.

352. Bitterman $P$, Rennard $S$, Adelberg S, Crystal RG. Role of fibronectin in fibrotic lung disease. A growth factor for human lung fibroblasts. Chest 1983;83:96S.

353. Brown LA, Ritzenthaler JD, Guidot DM, Roman J. Alveolar type II cells from ethanol-fed rats produce a fibronectin-enriched extracellular matrix that promotes monocyte activation. Alcohol 2007 Aug;41(5):317-324. 


\section{ABBREVIATIONS}

ADAMTs disintegrin and metalloproteinase with thrombospondin motif

$\mathrm{ADH} \quad$ Alcohol dehydrogenase

ALD Alcoholic liver disease

ALI Acute lung injury

AMPB Alpha-1-microglobulin/bikunin precursor

ALT Alanine aminotransferase

ARDS Acute respiratory distress syndrome

As Arsenic

ASH Alcoholic steatohepatitis

AST Aspartate aminotransferase

BAL(F) Bronchioalveolar lavage (fluid)

BSA Bovine serum albumin

BW Body weight

CAE Chloroacetate esterase

CID Collision induced dissociation

CRELD2 Cysteine-rich with EGF-like domain protein 2

CYP2E1 Cytochrome P450 2E1

ECM Extracellular matrix

Etan Etanercept

ETD Electron transfer dissociation

EtOH Ethanol

FA Formic acid

GAPDH Glyceraldehyde 3-phosphate dehydrogenase 


\begin{tabular}{|c|c|}
\hline GI & Gastrointestinal \\
\hline $\mathrm{GnHCl}$ & Guanidine Hydrochloride \\
\hline GO & Gene ontology \\
\hline$H \& E$ & Hematoxylin \& eosin \\
\hline HFD & High fat diet \\
\hline HMGB1 & High mobility group protein B1 \\
\hline HSCs & Hepatic stellate cells \\
\hline i.p. & intraperitoneal \\
\hline IL-10 & Interlukin-10 \\
\hline IL-1b & Interleukin-1 $\beta$ \\
\hline IL-2 & Interleukin-2 \\
\hline IL-6 & Interleukin-6 \\
\hline IL-8 & Interleukin-8 \\
\hline KC & Keratinocyte chemoattractant \\
\hline LC & Liquid chromatography \\
\hline LefSe & Linear effect size \\
\hline LFD & Low fat diet \\
\hline LPS & Lipopolysaccharide \\
\hline LW & Liver weight \\
\hline MD & Maltose-dextrin \\
\hline MIP-2 & Macrophage inflammatory protein \\
\hline MMPs & Matrix metalloproteinases \\
\hline MS & Mass spectroscopy \\
\hline $\mathrm{NaCl}$ & Sodium chloride \\
\hline NAFLD & Non-alcoholic fatty liver disease \\
\hline $\mathrm{NASH}$ & Non-alcoholic steatohepatitis \\
\hline NEFA & Non-esterified fatty acid \\
\hline OFC & Oligofructose \\
\hline OTU & Operational taxonomic unit \\
\hline
\end{tabular}


PAl-1 Plasminogen activator inhibitor

PCOA Principle coordinate analysis

PEBP1 Phosphatidylethanolamine-binding protein

RANTES Regulated on activation, normal T cell expressed and secreted

SAA1 Serum amyloid A1

SAA2 Serum amyloid A2

SDS Sodium dodecyl sulfate

TG Triglycerides

TGF $\beta \quad$ Transforming growth factor beta

TIMP Tissue inhibitor of matrix metalloproteinases

TNF $\alpha \quad$ Tumor necrosis factor-alpha 


\author{
CURRICULUM VITAE \\ Veronica L. Massey \\ University of Louisville School of Medicine \\ Department of Pharmacology and Toxicology \\ Louisville, KY 40292 \\ vlmass01@louisville.edu \\ 502-852-5157
}

\title{
EDUCATION
}

2010-2012 M. S. in Pharmacology \& Toxicology, University of Louisville, Louisville, KY

2006-2010 B.S. in Biology, Xavier University, Cincinnati, OH

\section{PROFESSIONAL EXPERIENCE}

2012-2014 Graduate Research Fellow, University of Louisville, Department of Pharmacology \& Toxicology

2010-2011 Graduate Research Assistant, University of Louisville, Department of Pharmacology \& Toxicology

2009-2010 Lab assistant, Cincinnati Children's Hospital, Department of Developmental Biology

2008-2009 Undergraduate researcher, University of Louisville, Department of Pharmacology \& Toxicology

\section{PROFESSIONAL MEMBERSHIPS AND ACTIVITIES}

2013-present Society of Toxicology, Student member

2013-present Ohio Valley Society of Toxicology

2010-present University of Louisville Alcohol Research Center 


\section{HONORS AND AWARDS}

12/2014 The Dean's Citation

09/2014 $\quad 1^{\text {st }}$ Place Poster Award at the NIEHS Tamborro Symposium

09/2013 Battelle Minority/Women Travel Award for the 2013 Society of Toxicology Meeting

09/2013 Invitation for Platform Presentation, Ohio Valley Society of Toxicology Regional Meeting, University of Louisville, Louisville, KY

09/2013 School of Medicine Research Committee Travel Award

09/2013 Graduate Student Council Travel Award

2008-2010 Member of Alpha Epsilon Delta Honors Society, Xavier University

2006-2010 Xavier University Academic Scholarship

2006-2010 Dean's List, Xavier University

\section{SERVICE}

Service to Department

01-04/2014 Pharmacology and Toxicology Admissions Committee This committee serves as the committee who reviews interviews applicants to the departments master's and doctoral programs. I served as a student representative and took part in review of applications and interview with applicants. As the student representative, I was also responsible for of hosting students during their stay in Louisville.

2013-2014 Pharmacology and Toxicology Planning Committee This meets as needed to plan department events including the annual summer picnic and the annual holiday party. I served as the student representative.

2012-2014 Pharmacology and Toxicology Student Affairs Committee This committee met as needed to discuss department curriculum, requirements, and student concerns. As one of the student 
representative, I served as a liaison and an advocate for the student body.

\section{Service to the Institution}

08/2014 Professional Development (PLAN) workshop panelist This workshop, titled Managing life as a Graduate student, included a panel of 4 graduate students who engaged in a discussion with new graduate students regarding time management and professional development during graduate school. I served as a panelist, representing the School of Medicine's graduate program.

2013-2014 University of Louisville School of Medicine Website Advisory Committee

This student committee was organized to aid in the organization of the School of Medicine's updated website. I served as a graduate student representative to voice the needs and concerns of the graduate students and their programs during the website's design.

2013 Educational Enterprise Committee, Scholarship Subcommittee This committee was established as part of the $21^{\text {st }}$ Century University initiative. Over the course of several months, this committee met weekly or bi-weekly to produce a plan that clarified the School of Medicine's vision for educational excellence in the areas of scholarship, service, and curriculum. I served as a graduate student representative to the committee as well as a member of the Scholarship subcommittee.

2012-2013 Vice Dean of Research Search Committee This committee was charged with the task of identifying a list of semifinal candidates for the position of Vice Dean of Research. I served as the graduate student representative to the committee. As such, I took an active part in the reviewing of applications as well as applicant interviews.

08/2013 Integrated Program in Biomedical Sciences Student Panelist

10/2011 Judging assistant, Research!Louisville

10/2010 Judging assistant, Research!Louisville 


\section{Service to the Community}

03/2013 Head Judge, Cellular and Molecular Biology, Manual High School Science Fair

02/2013 Judge, St. Patrick's School Science Fair

03/2012 Judge, Cellular and Molecular Biology, Manual High School Science Fair

\section{EDUCATION ACTIVITIES}

\section{Lectures/labs}

3/14 Guest lecturer (Alcohol Pharmacology), Basics of Pharmacology, University of Louisville

9/13 Facilitator for Autonomic Pharmacology Simulation Lab, Pharmacology, University of Louisville School of Medicine

3/13 Teaching Assistant and lecturer (Alcohol Pharmacology), Basics of Pharmacology, University of Louisville

\section{Professional Development}

2013-2014 Graduate Teaching Assistant Academy

\section{LEADERSHIP}

2013-2014 Graduate Student Council Representative for the Department of Pharmacology \&Toxicology

2013-1014 President, Pharmacology \&Toxicology Graduate Student Organization

2012-2013 Vice President, Pharmacology \& Toxicology Graduate Student Organization

2011-2012 Secretary, Pharmacology \& Toxicology Graduate Student Organization, University of Louisville

2010-2014 Class Representative, Department of Pharmacology \& Toxicology, University of Louisville 
2009-2010 Secretary, Biology Club, Xavier University

\section{FUNDING}

2011-present NIEHS T-32 Training Grant

2010-2011 Graduate Research Fellowship, Integrated Programs in Biomedical Sciences

\section{ABSTRACTS AND PRESENTATIONS}

\section{ORAL PRESENTATIONS}

\section{Local/Regional}

1. Research Seminar 03/14 The role of transitional ECM in the parallel and interdependent mechanisms of liver and lung injury. University of Louisville Alcohol Research Center, University of Louisville, Louisville, KY

2. Platform Presentation, 09/13, The prebiotic oligofructose protects against liver injury caused by arsenic in a model of NASH. Ohio Valley Society of Toxicology, University of Louisville, Louisville, KY

3. Research Seminar, 11/12, Parallel and interdependent mechanism of alcohol-induced organ injury in the liver and lung. University of Louisville Alcohol Research Center, Louisville, KY

4. Research Seminar, 8/11 OVSOT Summer Meeting. CycloRGDfV protects against LPS-induced liver injury caused by ethanol in mice.

5. Research Seminar, 03/11, Integrin inhibitor Cyclo-RGDfV blunts enhanced LPS-induced liver injury caused by ethanol in mice. University of Louisville, Seminar in Pharmacology and Toxicology, Louisville, KY.

\section{POSTERS}

\section{National}

1. Poster Presentation 09/14, The prebiotic oligofructose protects against enhanced liver injury caused by arsenic in a model of NASH, Tamburro Symposium on Environmental Chemicals and Liver Disease, Louisville, KY 
2. Poster Presentation $3 / 14$ The prebiotic oligofructose protects against enhanced liver injury caused by arsenic in a model of NASH, Society of Toxicology, Phoenix, AZ

3. Poster Presentation 11/13, The prebiotic oligofructose protects against enhanced liver injury caused by arsenic in a model of NASH, American Association for the Study of Liver Disease, Washington D.C.

4. Poster Presentation, 11/11, Integrin inhibitor Cyclo-RGDfV blunts enhanced LPS-induced liver injury caused by ethanol in mice. American Association for the Study of Liver Disease, San Francisco, CA.

\section{Local/Regional}

1. Poster Presentation 09/14, Characterization of the liver:lung axis in alcoholinduced lung damage, Research!Louisville, Louisville, KY

2. Poster Presentation 09/13, The prebiotic oligofructose protects against enhanced liver injury caused by arsenic in a model of $\mathrm{NASH}$, Research!Louisville, Louisville, KY

3. Poster Presentation, 10/12, Integrin $\beta 3^{-/-}$mice are protected against enhanced alcohol-induced liver injury. Research!Louisville Louisville, KY

4. Poster Presentation, 10/11, Integrin av $\beta 3$ mediates ethanol-enhanced liver damage caused by LPS. Research!Louisville, Louisville, KY.

5. Poster Presentation, 10/11, Ethanol sensitization to LPS-induced liver injury: protection by the integrin inhibitor, cycloRGDfV. Ohio Valley Society of Toxicology Regional Meeting, Dayton, $\mathrm{OH}$.

\section{ABSTRACTS}

\section{National}

1. Massey VL, Poole LG, Torres E, Dolin C, Merchant ML, Ritzenthaler J, Roman J, Arteel GE. (2014) Characterization of the liver:lung axis in alcohol-induced lung damage. Hepatology 59 (S1) 616

2. Anders LC, Douglas AN, Mohammad MK, Massey VL, Kirpich IA, Falkner KC, Warner NL, Arteel GE, Cave M, McClain CJ and Beier JI (2014) Vinyl Chloride Predisposes The Liver To Injury: Implications For Human Exposure. Gastroenterology 146(5):S910. (Selected for oral presentation).

3. Massey VL, Schmidt RH, Tan M, Watson WH, Arteel GE. (2014) The prebiotic oligofructose protects against enhanced liver injury caused by 
arsenic in a model of NASH The Toxicologist. Supplement to Toxicological Sciences 138(1) 1323

4. Anders L, Douglas A, Guardiola J, Massey VL, Kirpich I, Mohammad M, Falkner KC, Cave M, McClain C, Beier J. (2014) An environmental $2^{\text {nd }}$ hit: effect of vinyl chloride exposure on liver injury in experimental hepatotoxicity and in humans. The Toxicologist. Supplement to Toxicological Sciences. 138(1) 1879

5. Massey VL, Schmidt RH, Tan M, Watson W, Arteel GE (2013) The prebiotic oligofructose protects against enhanced liver injury caused by arsenic in a model of NASH. Hepatology 58 (S1) 739A

6. Schmidt RH, Massey VL, Jokinen J, Falkner KC, Beier JI, Arteel GE (2013) Olanzapine-induced liver injury: direct metabolic effects on liver and exacerbation by high-fat diet. Hepatology 58 (S1) 704A

7. Schmidt RH, Tan M, Jokinen J, Massey VL, Zhong H, Arteel GE (2012) Arsenic enhances experimental liver disease via altering $\mathrm{Gl}$ tract function: protection with prebiotics. Hepatology 56 (S1): 89A

8. Massey VL, Beier JI, Schmidt RH, Zhong H, Arteel GE (2011) Integrin inhibitor Cyclo-RGDfV blunts enhanced LPS-induced liver injury caused by ethanol in mice. Hepatology 54(S1):970A

9. Tan M, Massey VL, Ding X, Zhong H, States JC, and Arteel GE (2010) Arsenic pre-exposure delays cell cycle entry and progression in mouse liver after partial hepatectomy. Hepatology 52:462A

\section{Local/Regional}

1. Massey VL, Poole LG, Torres E, Dolin C, Merchant ML, Ritzenthaler J, Roman J, Arteel GE. (2014) Characterization of the liver:lung axis in alcohol-induced lung damage. Research!Louisville. Louisville, KY.

2. Christine DE, Massey, VL, Poole, LG, Siow, DL,Merchant, ML, Wilkey, DW, Arteel,GE. (2014) The hepatic 'matrisome' responds dynamically to stress: novel characterization of the ECM proteome. Research!Louisville. Louisville, KY.

3. Watson W, Burke T, Massey VL, Arteel GE, Merchant ML (2014) Activity of the zinc finger transcription factor HNF-4a is inhibited by arsenic in the livers of mice with diet induced non-alcoholic fatty liver disease. Research!Louisville. Louisville, KY. 
4. Poole LG, Massey VL, Dolin C, Merchant M, Beier JI, Roman J, Arteel GE (2014) Ethanol-induced changes in the hepatic ECM directly enhance the pro-inflammatory response of macrophages. Research!Louisville. Louisville, KY.

5. Bushau, A, Anders L, Douglas A, Poole LG, Massey VL, Lang A, Falkner C, Cave M, McClain C, Beier J (2014) Mechanistic insights into vinyl chloride-induced liver injury. Research!Louisville. Louisville, KY.

6. Massey VL, Schmidt RH, Tan M, Watson WH, Arteel GE (2013) The prebiotic oligofructose protects against enhanced liver injury caused by arsenic in a model of NASH. Ohio Valley Society of Toxicology Annual Meeting, Louisville KY

7. Massey VL, Schmidt RH, Tan M, Watson WH, Arteel GE (2013) The prebiotic oligofructose protects against enhanced liver injury caused by arsenic in a model of NASH. Research!Louisville

8. Poole LG, Jokinen JD, Massey VL, Beier JI, and Arteel GE (2013) Sinusoidal endothelial cell-derived extracellular matrix regulates basal and stimulated macrophage activation. Research!Louisville.

9. Massey VL, Poole LG, Schmidt RH, Arteel GE (2012) Integrin $\beta 3^{-/-}$mice are protected against enhanced alcohol-induced liver injury.

Research!Louisville Louisville, KY

10. Massey VL, Beier JI, Schmidt RH, Zhong H, Arteel GE (2011) Ethanol sensitization to LPS-induced liver injury: protection by the integrin inhibitor, cycloRGDfV. Ohio Valley Society of Toxicology Annual Meeting, Dayton, Ohio

11. Massey VL, Beier JI, Schmidt RH, Zhong H, Arteel GE (2011) Integrin av $\beta 3$ mediates ethanol-enhanced liver damage caused by LPS.

Research!Louisville Louisville, KY

\section{PUBLICATIONS}

\section{Papers}

1. Massey VL, Dolin C, Poole LG, Wilkey D, Merchant ML, Torres E, Roman J, Arteel GE. The hepatic 'matrisome' responds dynamically to stress: novel characterization of the ECM proteome (in preparation) 
2. Massey VL, Torres E, Poole LG, Schmidt RH, Ritzenthaler J, Roman J, Arteel GE Characterization of a model of concomitant liver and lung injury in a 2-hit model of chronic alcohol exposure (in preparation)

3. Massey VL, Schmidt RH, Tan M, Stocke K, Neal RE, Barve S, Arteel GE. The prebiotic oligofructose protects against arsenic-enhanced liver injury in a model of NAFLD via modulation of the gut microbiome (in preparation)

4. Schmidt RH, Jokinen JD, Massey VL, Falkner KC, Shi X, Yin X, Zhang X, Beier JI, Arteel GE (2013) Olanzapine activates hepatic mammalian target of rapamycin (mTOR): New mechanistic insight into metabolic dysregulation with atypical antipsychotic drugs. J Pharmacol Exp Ther

5. Massey VL and Arteel GE (2012). Acute alcohol-induced liver injury. Frontiers in Physiology. 3 (193) 1-8

\section{Book Chapters}

1. Arteel GA, Massey VL, Ritzenthaler JD, Roman JR. The Gut/Liver/Lung axis and alcohol-induced-tissue pathology. 